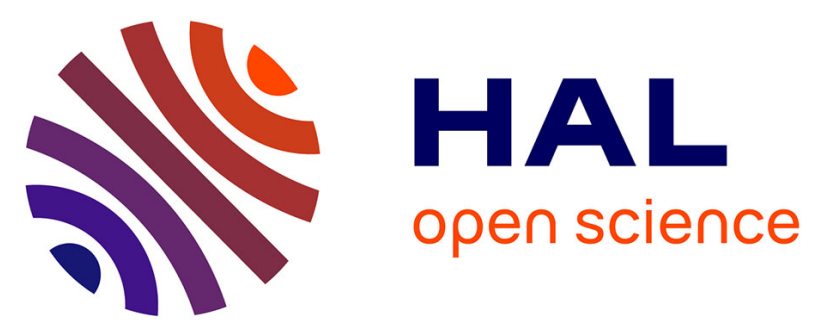

\title{
Coordinated Hubble Space Telescope and Venus Express Observations of Venus' upper cloud deck
}

\author{
Kandis Lea Jessup, Emmanuel Marcq, Franklin Mills, Arnaud Mahieux, \\ Sanjay Limaye, Colin Wilson, Mark Allen, Jean-Loup Bertaux, Wojciech \\ Markiewicz, Tony Roman, et al.
}

\section{To cite this version:}

Kandis Lea Jessup, Emmanuel Marcq, Franklin Mills, Arnaud Mahieux, Sanjay Limaye, et al.. Coordinated Hubble Space Telescope and Venus Express Observations of Venus' upper cloud deck. Icarus, 2015, 258, pp.309-336. 10.1016/j.icarus.2015.05.027 . insu-01164292

\section{HAL Id: insu-01164292 \\ https://hal-insu.archives-ouvertes.fr/insu-01164292}

Submitted on 4 Mar 2021

HAL is a multi-disciplinary open access archive for the deposit and dissemination of scientific research documents, whether they are published or not. The documents may come from teaching and research institutions in France or abroad, or from public or private research centers.
L'archive ouverte pluridisciplinaire HAL, est destinée au dépôt et à la diffusion de documents scientifiques de niveau recherche, publiés ou non, émanant des établissements d'enseignement et de recherche français ou étrangers, des laboratoires publics ou privés. 


\section{Coordinated Hubble Space Telescope and Venus Express Observations of Venus' upper cloud deck}

Authors: Kandis Lea Jessup ${ }^{1,3}$, Emmanuel Marcq ${ }^{2}$, Franklin Mills ${ }^{3,10}$, Arnaud Mahieux ${ }^{4}$, Sanjay Limaye ${ }^{5}$, Colin Wilson ${ }^{6}$, Mark Allen ${ }^{7}$, Jean -Loup Bertaux ${ }^{2}$, Wojciech Markiewicz ${ }^{8}$, Tony Roman ${ }^{9}$, Ann-Carine Vandaele ${ }^{4}$, Valerie Wilquet ${ }^{4}$, Yuk Yung ${ }^{8}$

Southwest Research Institute, 1050 Walnut Street, Boulder CO 80302, USA ${ }^{1}$ Université de Versailles Saint-Quentin - Laboratoire LATMOS, 11 boulevard d'Alembert, 78280 Guyancourt. France ${ }^{2}$

Research School of Physics and Engineering and Fenner School of Environment and Society, the Australian National University, Canberra, ACT $0200^{3}$

Belgian Institute for Space Aeronomy, Avenue Circulaire, 3, B-1180 Brussels, Belgium $^{4}$

University of Wisconsin, Atmospheric Oceanic \&Space Science Department, 1225 W Dayton St Madison WI, 53706 USA $^{5}$

Oxford University, Oxford, $\mathrm{UK}^{6}$

Caltech, 1200 E California Blvd Pasadena, CA, 91125, USA ${ }^{8}$

Max Planck Institute for Solar System Research, Justus-von-Liebig-Weg 3, 37077 Göttingen, Germany ${ }^{7}$

Space Telescope Science Institute, 3700 San Martin Drive Baltimore MD 21218, $\mathrm{USA}^{9}$

Space Science Institute, 4750 Walnut St \#205, Boulder CO 80301, USA ${ }^{10}$

Pages: 54 (1.5 spaced, including 5 pages of references)

Figures: 19

Tables: 5 


\begin{abstract}
Hubble Space Telescope Imaging Spectrograph (HST/STIS) UV observations of Venus' upper cloud tops were obtained between 20N and 40S latitude on December 28, 2010; January 22, 2011 and January 27, 2011 in coordination with the Venus Express (VEx) mission. The high spectral $(0.27 \mathrm{~nm})$ and spatial (40-60 km/pixel) resolution HST/STIS data provide the first direct and simultaneous record of the latitude and local time distribution of Venus' $70-80 \mathrm{~km} \mathrm{SO}$ and $\mathrm{SO}_{2}\left(\mathrm{SO}_{\mathrm{X}}\right)$ gas density on Venus' morning quadrant. These data were obtained simultaneously with a) VEx/SOIR occultation and/or ground-based James Clerk Maxwell Telescope sub-mm observations that record respectively, Venus' near-terminator $\mathrm{SO}_{2}$ and dayside $\mathrm{SO}_{\mathrm{x}}$ vertical profiles between $\sim 75-100 \mathrm{~km}$; and b) $0.36 \mu \mathrm{m} \mathrm{VEx} / \mathrm{VMC}$ images of Venus' cloud-tops. Updating the (Marcq, E., Belyaev, B., Montemssin, F., Fedoroav, A., Bertuax, J-L., Vandaele, A. C., Neefs, E. [2011], Icarus, 211: p. 58-69) radiative transfer model $\mathrm{SO}_{2}$ gas column densities of $\sim 2-10$ $\mu \mathrm{m}$-atm and $\sim 0.4-1.8 \mu \mathrm{m}$-atm are retrieved from the December 2010 and January 2011 HST observations, respectively on Venus' dayside (i.e., at Solar Zenith Angles $(\mathrm{SZA})<60^{\circ}$ ); SO gas column densities of 0.1$0.11 \mu \mathrm{m}$-atm, $0.03-0.31 \mu \mathrm{m}$-atm and 0.01-0.13 $\mu \mathrm{m}$-atm are also retrieved from the respective December 28, 2010, January 22, 2011 and January 27, 2011 HST observations. A decline in the observed low-latitude 0.24 and $0.36 \mu \mathrm{m}$ cloud top brightness also paralleled the declining $\mathrm{SO}_{\mathrm{x}}$ gas densities. On December 28, $2010 \mathrm{SO}_{2}$ VMR values $\sim 280-290 \mathrm{ppb}$ are retrieved between 74-81 km from the HST and SOIR data obtained near Venus' morning terminator (at SZAs equal to $70^{\circ}$ and $90^{\circ}$, respectively); these values are $10 \mathrm{x}$ higher than the HST-retrieved January 2011 near terminator values. Thus, the cloud top $\mathrm{SO}_{2}$ gas abundance declined at all local times between the three HST observing dates. On all dates the average dayside $\mathrm{SO}_{2} / \mathrm{SO}$ ratio inferred from HST between $70-80 \mathrm{~km}$ is higher than that inferred from the sub-mm the JCMT data above $84 \mathrm{~km}$ confirming that $\mathrm{SO}_{\mathrm{x}}$ photolysis is more efficient at higher altitudes. The direct correlation of the $\mathrm{SO}_{\mathrm{x}}$ gases provides the first clear evidence that $\mathrm{SO}_{\mathrm{x}}$ photolysis is not the only source for Venus' $70-80$ $\mathrm{km}$ sulfur reservoir. The cloud top $\mathrm{SO}_{2}$ gas density is dependent in part on the vertical transport of the gas from the lower atmosphere; and the $0.24 \mu \mathrm{m}$ cloud top brightness levels are linked to the density of the submicron haze. Thus, the new results may also suggest a correlation between Venus' cloud-top sub-micron haze density and the vertical transport rate. These new results must be considered in models designed to simulate and explore the relationship between Venus' sulfur chemistry cycle, $\mathrm{H}_{2} \mathrm{SO}_{4}$ cloud formation rate and climate evolution. Additionally, we present the first photochemical model that uniquely tracks the transition of the $\mathrm{SO}_{2}$ atmosphere from steady to non-steady state with increasing SZA, as function of altitude within Venus' mesosphere, showing the photochemical and dynamical basis for the factor of $\sim 2$ enhancements in the $\mathrm{SO}_{\mathrm{x}}$ gas densities also observed by HST near the terminator above that observed at smaller SZA. These results must also be considered when modeling the long-term evolution of Venus' atmospheric chemistry and dynamics.
\end{abstract}

Keywords: Venus; Venus, atmosphere; Atmospheres, composition; Atmospheres, chemistry 


\section{Introduction and Overview}

Venus' atmosphere is known to be composed predominantly of carbon dioxide (total volume mixing ratio of 0.965 ) and nitrogen gas, where the latter is a distant second to the former. Although sulfur oxide gases and aerosols are only trace components of Venus' atmosphere, chemical reactions in Venus' atmosphere that involve these species such as $\mathrm{SO}_{2}, \mathrm{SO}, \mathrm{OCS}$, and $\mathrm{H}_{2} \mathrm{SO}_{4}$, are important because they are closely linked to the global-scale $\mathrm{H}_{2} \mathrm{SO}_{4}$ cloud and haze layers located at altitudes between 30-100 km (Fig. 1). Additionally, though volcanic activity has yet to be directly observed on Venus, detailed thermochemical modeling of Venus' near surface chemical make-up indicates that volcanic outgassing is the most probable source atmospheric sulfur in Venus' lower atmosphere (i.e. below $60 \mathrm{~km}$ ) (Johnson and Fegley 2002).

Notably, Venus' 60-100 km altitude region was extensively observed during the time period extending from the late 1970s to early 1990s and during the Venus Express (VEx) mission (2006-2014). Likewise, extensive modeling of the dynamics and chemistry of Venus’ atmosphere (e.g., Krasnopolsky and Pollack 1994, Krasnopolsky 2007) has been on-going. Yet, the mechanism(s) that control the exchange of $\mathrm{SO}_{2}$ between the lower atmosphere and the mesosphere are not fully understood. Some recent modeling efforts have considered whether the vertical transport of the gas to the mesosphere occurs in conjunction with Hadley cell circulation (Yung et al 2009, Marcq et al. 2013); while previous observers have suggested that direct volcanic ejection (Esposito et al 1988) may be a key mechanism for the exchange of the gas from the troposphere $(\mathrm{z}<60 \mathrm{~km})$ to the mesosphere $(\sim 60-90 \mathrm{~km}$, see Clancy et al. 2003, Betraux et al. 2007). The biggest challenge to understanding how the exchange occurs is the fact that portions of the lower atmospheric region are statically stable. Suppression of this stability due to changes in the vertical temperature profile may help promote the vertical transport of sulfur, and other processes such as small scale eddies, and or adsorption/desorption on cloud particles (just to name a few) may also contribute to the vertical transport of sulfur in Venus' atmosphere. Because the exact mechanism for the vertical transport of sulfur is unknown sulfur oxide observations are highly prized since they provide the data needed to properly assess the ongoing chemical evolution of Venus' 
atmosphere, atmospheric dynamics, and could even provide insight into the nature and frequency of volcanism on Venus.

Consequently, a vast suite of $\mathrm{SO}_{2}$ and $\mathrm{SO}$ observations were obtained in conjunction with the VEx mission (e.g., Sandor et al 2010, Belyaev et al 2012, Marcq et al 2011) in order to probe the 60-100 km altitude region of Venus' atmosphere using both Earth-based telescopes and VEx instrumentation. These observations have revealed unexpected spatial patterns and spatial/temporal variability that have not been satisfactorily explained by models (Zhang et al 2012, Krasnopolsky 2012). For example, high ( $\geq 400 \mathrm{ppb}$ ) $\mathrm{SO}_{2}$ abundances were observed within the mesosphere in 2007 (Belyaev et al 2012, Marcq et al 2011), and an on-going decline in the $\mathrm{SO}_{2}$ abundance was consistently observed throughout the remainder of the VEx mission (Marcq et al. 2013, Marcq pers. communication) [Fig. 2]. Venus' long-term (i.e., from 1980 to 2014) mesospheric $\mathrm{SO}_{2}$ abundance record suggests this behavior may be cyclical in nature (Marcq et al 2013); if so, the observed behavior cannot be explained as a function of local time variations but may be an indication of active volcanism. Of equal intrigue is the equator-to-pole $\mathrm{SO}_{2}$ abundance gradient, which seems to be typically increasing towards the equator, but can also show the opposite trend (Marcq et al. 2013). The mechanism that causes the observed latitudinal gradient to change slope is not fully understood, but seems to be connected with low levels of $\mathrm{SO}_{2}$ gas abundance, and may be connected to changes in the convective mixing intensity (c.f. Marcq et al. 2013).

In December 2010 and January 2011 170-310 nm, high spectral (0.3 nm) and spatial (40-60 km/pixel) resolution observations of Venus' low latitude dayside atmosphere were obtained using Hubble’s Space Telescope Imaging Spectrograph (the HST/STIS). These observations probe the $\mathrm{SO}_{2}$ and $\mathrm{SO}$ gas absorption signature in Venus' $\sim 65-80 \mathrm{~km}$ region and were obtained in coordination with (a) low latitude Venus Express Monitoring Camera (VMC) cloud top imaging; (b) solar occultation $\mathrm{SO}_{2}$ spectroscopy obtained using the VEx Solar Occultation in the Infrared (VEx/SOIR), (Belyaev et al. 2012); (c) and ground-based sub-mm measurements of Venus's average dayside SO and $\mathrm{SO}_{2}$ gas densities between 70-100 km obtained using the James Clerk Maxwell Telescope (JCMT) (Sandor et al. 2010). The acquired HST/STIS data also serve as a complement to the high-spatial, low-spectral $(1.5 \mathrm{~nm})$ resolution nadir viewing spectra obtained using 
the VEx Spectroscopy for Investigation of Characteristics of the Atmosphere of Venus (VEx/SPICAV) (Macrq et al. 2013) throughout the lifetime of the Venus Express mission. The primary objective of the coordinated observing effort was to obtain detailed information on the horizontal distribution of the $\mathrm{SO}_{2}$ and $\mathrm{SO}$ gas density in the 65-80 km region, while a) simultaneously obtaining information on the vertical distribution of the gases between 65-100 km; and b) collecting information on the cloud top brightness levels in the regions that are spatially co-located with the $\mathrm{SO}_{2}$ and $\mathrm{SO}$ gas density measurements. These types of data are needed to develop observationally informed models that are able to simulate and explore the photochemical, dynamical and radiative processes responsible for Venus’ atmospheric evolution.

\section{Observation Description}

The first (and thus far only) HST/STIS observations of Venus' upper cloud deck were obtained on 3 dates in December 2010 and January 2011 using the G230LB (170$317 \mathrm{~nm}$ ) grating, with the 52 "x0.1" slit and the CCD detector ( $0.51 "$ pixels), to obtain high spectral $(0.27 \mathrm{~nm})$ and spatial (40-60 km/pixel, assuming 2 pixel binning) resolution measurements of Venus' $\mathrm{SO}_{2}$ and $\mathrm{SO}$ gas absorption signatures [Fig. 3]. The specific dates of observation were December 28, 2010; January 22, 2011; and, January 27, 2011. On each date (or "visit" to Venus) Venus was observed with the 52" HST/STIS slit centered on the morning terminator longitude, so that Venus' morning quadrant was observed from the terminator to the sunlit limb. For our chosen dates the observable sunlit quadrant corresponded to $\sim 12-18 "$ of daylight, covering $\sim 90-121^{\circ}$ E. longitude. And, on each date Venus was observed with the HST/STIS slit in its nominal $45^{\circ}$ orientation. In this orientation, a different time of day and latitude was recorded in each pixel. In order to build up a picture of the $\mathrm{SO}_{2}$ and $\mathrm{SO}$ gas absorption signatures as a function of time of day at multiple latitudes, two exposures were taken on each visit, and in each exposure the center of the 52" slit intersects the morning terminator longitude at a single unique latitude [Fig. 4, Table I].

On each date, the data were obtained simultaneously and/or contemporaneously with ground-based sub-mm spectral observations of Venus and UV-Visible imaging of Venus' atmosphere obtained by the VEx/VMC and VEx/VIRTIS (Visible and Infrared 
Thermal Imaging Spectrometer) or near infrared spectral observations obtained with the VEx/SOIR instrument (see Table II). Additionally, our observing program was designed to align with the planned coordinated VEx and JAXA launched Venus Climate Orbiter (VCO, also known as Akatsuki) imaging of Venus' cloud tops-thus imaging from both an equatorial and pole-centered view would have been simultaneously obtained with the spectral observations. As alluded to above, the primary objective of the coordinated observing effort was to obtain detailed information on the horizontal distribution of Venus' cloud top $\mathrm{SO}$ and $\mathrm{SO}_{2}$ gas abundance distribution (in terms of latitude, longitude and time of day), while simultaneously obtaining information on the vertical distribution of the gases between $65-100 \mathrm{~km}$, as well as collecting information on the cloud contrast and/or brightness in the regions that were spatially co-located with the $\mathrm{SO}_{2}$ and $\mathrm{SO}$ gas density measurements. Although VCO was not able to successfully insert into Venus Orbit in December 2010, the coordinated VEx and earth based observing effort was executed.

Thus, on December 28, 2010, VEx/SOIR $4 \mu \mathrm{m}$ solar occultation spectra of Venus' mesosphere were obtained along the Earth-facing morning terminator at 2 UT at an impact (or target) latitude of $30.68 \mathrm{~S}$ [Fig. 5]. In coordination with the VEx/SOIR observations, HST/STIS observations of Venus that recorded Venus' dayside 170-310 nm UV signature from noon to the Earth-facing morning terminator were obtained within the 0-2 UT time period. In the first exposure [OBS 0] the target latitude along the Earth facing terminator was defined to be $15 \mathrm{~S}$ latitude. In the second exposure [OBS 1], taken simultaneously with the VEx/SOIR occultation observations, the target latitude at the terminator was set at $32 \pm 2 \mathrm{~S}$ latitude in order to match the target latitude of the VEx/SOIR solar occultation observations. In conjunction with the SOIR observations, observations of Venus' airglow were obtained by Venus Express using the VIRTIS-M instrument, which covers the near-UV and visible wavelengths from 0.25-1.0 $\mu \mathrm{m}$. However, these observations focused on the nightside near the anti-solar point. While these observations can provide data needed to better understand the day vs. night oxygen abundances on Venus, an in-depth discussion of the implications of the nightside VIRTIS data relative to the morning quadrant HST observations is beyond the scope of this paper. 
On January 22 and 27, 2011 VCO would have been at an optimal position to obtain UV images of the Venus cloud tops on the dayside hemisphere observable from Earth-based telescopes in the time period extending from 13:00-19:00 UT. At the same time, the orbital phases of VCO and VEx would have been coincident. Therefore, VEx scheduled and acquired successive UV images of Venus' cloud tops using the VEx/VMC camera that could be used to calibrate and validate the planned VCO imaging sequence. As a complement to those observations, HST/STIS observations were obtained on each of these dates in the 15-18 UT time period. As discussed above, two HST/STIS exposures (OBS 2 \& OBS 3 on January 22 and OBS 4 \& OBS 5 on January 27) were obtained per day of observation. For each of these dates, the slit was centered at the morning terminator at $45 \mathrm{~S}$ in the first exposure and at 65S in the second exposure [Fig. 4]. Given the $45^{\circ}$ orientation of the slit, this resulted in the dayside coverage along the slit extending from high latitudes at the terminator to low latitudes at the sunlit limb [Fig. 4]. Because of the overlap in the time signature of the VMC imaging and the HST spectral observations, the $\mathrm{SO}_{2}$ gas density signature recorded in the HST spectra can be directly mapped to the cloud patterns captured in the field of view of the available VEx VMC images between 17-19 UT on January 22 and between 15-17 UT on January 27 [Fig. 5]. Additionally, because the HST/STIS observations were obtained in the exact same observing geometry on each of these dates, variability in the gas behaviour at each latitude and time of day was recorded at the cadence of Venus' 5 "day” cloud top rotation period. I.e., Titov et al. 2012 reports the cloud top zonal wind-speed to be $0.09 \mathrm{~km} / \mathrm{s}$ at latitudes $\pm 45^{\circ}$ from the equator; similarly, measurements provided by Khatuntsev et al. (2013) that bracket the time period of the coordinated observations show that the cloud top zonal wind speed was primarily $\sim 0.085-0.095 \mathrm{~km} / \mathrm{s}$, for an average zonal cloud top wind speed of $0.09 \mathrm{~km} / \mathrm{s}$. Given Venus' $6051 \mathrm{~km}$ radius it takes 4.89 Earth days to traverse Venus' circumference. The January 27 observations of Venus' cloud tops were obtained 4.92 Earth days subsequent to the January 22 observations, thus matching near exactly the expected equatorial cloud rotation period.

Based on the acquired HST/STIS observations we provide in Section 5 a detailed description of the $\mathrm{SO}_{2}$ and $\mathrm{SO}$ gas density variation as function of latitude and time of day for each of these dates. A description of the data calibration steps and the gas density 
retrieval methods is provided in Sections 3 and 4, respectively. And a detailed comparison of the HST results to the observations obtained contemporaneously by VEx as well as other ground-based observers is provided in Section 6

\section{Data Reduction}

3.1Flux Calibration: The HST/STIS observations were initially reduced using the Space Telescope Science Data Analysis System (STDAS) CALSTIS, i.e., the STIS calibration and reduction pipeline which handles flat-fielding, geometric rectification, bias and dark subtraction. However, the pipeline reduced spectra still retained some level of residual flux from the sky. Because the observations were obtained with the 52 " long slit, the sky background could be straightforwardly estimated from the flux levels recorded in regions of the slit sufficiently far from the edge of Venus' 22 \pm 2 " disk diameter. This value was then subtracted from the observed flux at all wavelengths.

Although HST/STIS has a solar blind Multi-Anode Microchannel Array (MAMA) detector, the UV flux from Venus would have exceeded the brightness levels of that detector. Consequently, in order to obtain high signal-to-noise $(\mathrm{S} / \mathrm{N})$ observations of Venus within the limited time period allotted for Venus observations, the exposures had to be obtained using the CCD detector and the G230LB grating, which is known to produce strong grating scattered light below $230 \mathrm{~nm}$ at wavelengths where the CCD detector has a high sensitivity. Fortunately, the grating scattered light levels can also be estimated directly from the observed spectra. The G230LB grating disperses light throughout the 170-320 nm region, but the solar flux becomes very weak below $200 \mathrm{~nm}-$ dropping by 3 orders of magnitude between 300 and $170 \mathrm{~nm}$ - and the expected signal from Venus below $190 \mathrm{~nm}$ is basically zero because of the strong $\mathrm{CO}_{2}$ absorption below $200 \mathrm{~nm}$. I.e., at $190 \mathrm{~nm}$ only about 1\% of incident solar flux is scattered back to the Earth from the cloud tops and that flux levels is about a factor of 2 below the $190 \mathrm{~nm}$ detection limit of the instrument when using G230LB grating in concert with the CCD detection; it is also a factor of 10 below the detection limit needed at $170 \mathrm{~nm}$. Consequently, any flux detected at wavelengths $<190 \mathrm{~nm}$ will be due to grating scattered light. Therefore, we used the flux levels detected at $185 \pm 5 \mathrm{~nm}$ to estimate the level of grating scattered light in 
the observed spectra and subtract off this residual flux from the observations at all wavelengths.

In each of these cases, to make the necessary corrections we first converted the calibrated flux defined per pixel in units of erg/s/ $\mathrm{A} / \mathrm{cm}^{2} / \operatorname{arcsec}^{2}$ to count rates based on the HST provided detector sensitivity levels per wavelength. Once the sky background and the grating scattered light levels were estimated in count units and subtracted off from the raw flux defined in count units, the observed flux was then returned to the calibrated flux units.

The CCD detector is also very sensitive to cosmic rays. Though the exposure times used for our observations are relatively short ( 0.5-2.5 min) the 2-D (1024 spatialpixel x 1024 spectral-pixel) spectral maps recorded by HST/STIS are riddled with cosmic rays. I.e., out of the 785 spectral pixels included in each spectrum above $200 \mathrm{~nm}$, there were $\sim 5 \pm 5$ spectral pixels that were impacted by cosmic rays per spatial pixel. Since Venus had not been observed by HST/STIS before, we opted to complete the cosmic ray correction of the data subsequent to the pipeline reduction of the data. Utilizing the corrected flux defined in count units, we removed the cosmic-rays from the spectral maps by comparing the count rate detected per spatial and spectral pixel with the median count rate detected within a 10 spatial pixel range, and replaced any high (more than 30x greater) count rates with the lower median value.

3.2 Wavelength Calibration: Analysis of the gas absorption signatures included in the Venus flux spectra was completed based on the ratio of the fully calibrated and corrected flux to a well calibrated solar spectrum. Consequently, although the initial pipeline reduction includes an approximate wavelength calibration, careful assessment of the absolute wavelength assignment per pixel post-pipeline reduction was needed to ensure alignment with the chosen solar spectrum. Because the variability of the highly structured solar spectrum at UV wavelengths can be significant, it was imperative that we utilize contemporaneously obtained solar spectra in the reduction of the HST acquired Venus spectra (McClintock et al. 2005, Egorova et al. 2008). We utilized the solar flux measured by SOLSTICE at (0.033 nm sampling, $0.1 \mathrm{~nm}$ FWHM) on each of the 6 dates of observation (Marty Snow, personal communication). To accurately align the solar spectrum and the observed Venus flux we re-sampled the SOLSTICE spectra to match 
the STIS data. In particular, the HST/STIS 0.1" slit and the G230LB grating (1.35 $\AA$ /pixel dispersion) with the CCD detector (which has $0.051^{\prime \prime} \mathrm{x} 0.051^{\prime \prime}$ pixels) produces data with a spectral resolution of $0.27 \mathrm{~nm}$ full width half maximum (FWHM) [Fig. 3]. Therefore, we convolved the SOLSTICE spectra with a Gaussian comparable to the complex $0.27 \mathrm{~nm}$ line-spread function (LSF) profile expected from the convolution of the STIS point-spread function (Woodgate 1998) with the $0.1^{\prime \prime}$ slit. We made small corrections to the wavelength of each STIS spectrum by aligning the Fraunhofer absorption lines with those in the solar spectrum. We found that adjustments of $0.09 \mathrm{~nm}$ or less (which is less than one-third of the FWHM) were required to achieve alignment with the solar spectrum. This step is a key safeguard against the introduction of artifacts into the reflectance spectra which is defined as a function of the ratio of the observed spectrum and the solar spectrum (see below for the complete definition of the reflectance spectrum).

3.3 Assignment of Spatial Coordinates per pixel: In order to assign the exact latitude, longitude, and time of day value to each spatial pixel we first generated a 2-D disk with a plate scale equivalent to the HST/STIS pixel size that is representative of the projection of Venus' Earth-facing disk on the sky. Based on this disk the latitude and longitude values at each point of the disk can be defined based on Venus' angular diameter on the date of observation and the known Venus North pole position angle. Likewise, we can project a 2-D representation of the slit across that disk based on the known Venus North pole position angle, the known HST/STIS spatial pixel size, the slit orientation relative to the North pole position angle and the target latitude and longitude of the center of the HST/STIS slit. Based on the intersection of that slit projection with the observable regions of Venus' Earth facing disk, the exact latitude, longitude, and time of day intercepted at each pixel along the length of the slit is defined. The accuracy of the latitude, longitude, and time of day assignments are thus dependent on accurate intersection of the center of the HST/STIS slit with the target latitude and longitude. To accommodate for any offsets between the targeted latitude and longitude and the observed latitude and longitude, we first identified the initial on-disk pixel value evidenced by the spatial profile produced by the observed flux and then adjusted the pixel assigned to the target longitude to the correspond to the value expected from the known 
geometry of the slit and the known radial extent of Venus' disk per date of observation. Because neither HST nor VEX obtained full disk images of Venus, the spatial profiles recorded in the HST/STIS slit could not be directly compared with a model profile derived from the disk image. Fortunately, the expected uncertainty in the HST/STIS targeting is $\sim 0.01$ arcsec (0.2 spatial pixel or $\sim 5 \mathrm{~km})$.

3.4 Spatial Binning: Once the latitude and longitude values had been assigned per pixel, we co-added the long-slit spectra along the length of the slit in order to improve the $\mathrm{S} / \mathrm{N}$ in each spatial bin. Initially, we co-added the data into spatial bins that were 2 spatial pixels long at each wavelength in order to retain a 0.1 " spatial resolution per spectral pixel, which is equivalent to $\sim 40-60 \mathrm{~km} /$ pixel depending on the date of observation. However, we found that variation in the reflectance levels recorded along the length of the slit from the terminator to the sunlit limb is relatively slow, such that the change in the observed reflectance levels over a 6 spatial pixel interval is negligible. Although binning the data every 6 spatial pixels reduces our spatial resolution to 0.3" /pixel (or $120-180 \mathrm{~km} /$ pixel), it also improves the $\mathrm{S} / \mathrm{N}$ of the data by 2 fold over that obtained when only 2 pixels are co-added, thus the 6 pixel spatial binning was adopted. Given that the slit encountered 14-18" of daylight per observation and that the pixels are 0.051" long, the 6 pixel binning segregates the spectral signature of Venus' cloud top reflectivity recorded by HST as a function of latitude and time of day into $53 \pm 5$ separate spatial bins per observation, depending on the date of observation.

Definition of the latitude, longitude, and time of day associated with the spatially re-binned spectra was straightforwardly determined by the boxcar average of the terms included in each of the co-added spatial bins.

3.5 Limb to Terminator Reflectance Spectra and Observational Trends: The reflectance $(R F)$ for each of the spatial bins is derived using the standard reflectivity relationship (c.f. Molvaerdikhani et al. 2012); i.e., it is defined as the ratio of the observed intensity $\left(F_{V}\right)$ multiplied by $\pi$ and divided by the solar flux at the earth $\left(F_{s}\right)$ that is scaled to Venus' heliocentric distance, thus:

$$
\mathrm{RF}=\left[\pi \times \mathrm{F}_{\mathrm{V}} \times R_{S}^{2}\right] \div\left[\mathrm{F}_{\mathrm{s}}\right]
$$


where $F_{V}$ is the fully calibrated and corrected flux recorded by HST/STIS, and $R_{S}$ is the heliocentric distance to Venus.

The highest $\mathrm{S} / \mathrm{N}$ levels are achieved at wavelengths longward of $210 \mathrm{~nm}$, therefore we show the reflectance spectra in the 210-310 nm region. As Figure 3 indicates, absorption from both the strong (180-240 nm, B-X transitions) and weak (280$310 \mathrm{~nm}, \mathrm{~A}-\mathrm{X}$ transitions) $\mathrm{SO}_{2}$ gas absorption band systems are evident in the data. However, we only see reliable evidence of the strong SO (190-240 nm, B-X transitions) gas absorption band; there is no clear evidence of absorption between 250-260 nm where absorptions due to the weak SO band transitions would be evidenced.

As discussed above the pixel-to-pixel spatial variance of the observed reflectance levels is slow, even when co-added at 6 spatial pixel intervals. Consequently, we find that we can adequately trace the variability of the atmosphere as a function of latitude and time of day by inverting the reflectance spectra derived at $\phi$ values (i.e., the angular distance from the subsolar longitude) ranging from $-15^{\circ}$ to $-70^{\circ}$ (i.e. for local times of 11 a.m to 7:15 a.m), at increments of $5^{\circ}$ longitude (or equivalently, on time intervals of 20 min on a 24 hour clock). For OBS0 \& OBS1 the subsolar longitude (local noon) is on the backside of the Earth facing disk; for OBS2 \& OBS3 the subsolar longitude is at the limb and is not truly discernable due to foreshortening, and although OBS4 \& OBS5 record the dayside reflectivity on the disk from noon to the terminator, for consistent comparison among observations (and to avoid foreshortened regions) we focus on the time period extending from 11 a.m. to 7:15 a.m.

Additionally, although in the all HST observations the slit was centered on the terminator in the southern hemisphere, so that data was recorded continuously from the terminator all the way to the sunlit limb (see Figs. 4 \& 5), in every case the signal recorded by HST at the terminator $\left(\phi=-90^{\circ}\right)$ is insufficient to obtain gas density measurements right at terminator. Notably, at the terminator the low-latitude solar zenith angle (SZA) is equal to $90^{\circ}$. Thus, we segregate our results into dayside (SZA $<60^{\circ}$ ) and near-terminator (SZA $\geq 60^{\circ}$ ) observations, and utilize the results derived from the high $\phi$ (high SZA) regions to understand trends near the terminator. Since HST reliably detects the cloud top flux for $\phi$ values $\sim-75 \pm 5^{\circ}$, due to the $45^{\circ}$ angle of the HST/STIS slit, on 
each date the latitude that intersects the STIS slit at $\phi=-75 \mathrm{dg}$ is always northward of the latitude of intersection at the terminator, i.e. at $\phi=-90$ (see Fig. 5, far-right panel). Consequently, all of the HST near-terminator observations were made within 40S latitude of Venus' equator, with the majority of the observations made within 30S latitude. While on the sunlit limb, the latitudes intersected closest to the edge of the disk (which is also closest to local noon, where $\phi=0^{\circ}$ ) extend from $10 \mathrm{~N}$ to $20 \mathrm{~N}$ latitude (see Fig. 5, far-left panel). Thus, nearly all the observations were made within $\pm 30^{\circ}$ latitude of Venus' equator.

\section{Gas Density Retrieval Methods}

The HST observations are analyzed utilizing the radiative transfer code developed by Marcq et al. (2011) for the analysis of the VEx/SPICAV nadir observations of Venus' atmosphere in the 200-300 nm range. A detailed description of the original code can be found in Marcq et al. (2011). The model uses the pseudo-spherical SPS-DISORT solver and assumes aerosol particles are spherical so extinction coefficients are calculated following Mie Theory. The ( $75 \mathrm{wt} \% \mathrm{H}_{2} \mathrm{SO}_{4}$ ) aerosol population is assumed to be bimodal. The radius of the smaller mode 1 , found in the upper haze layer, is $0.24 \mu \mathrm{m}$ and the radius of the larger mode 2, found in the upper cloud layer, is $1.1 \mu \mathrm{m}$.

We use the RT code to simulate Venus' reflectivity by defining 6 key elements: i) the atmospheric conditions defined in terms of pressure and temperature profiles; ii) the Rayleigh cross-sections of the dominant gas species, $\mathrm{N}_{2}$ and $\mathrm{CO}_{2}$; iii) the gas absorption cross-section (and temperature dependence) of the constituents that have strong absorptions within the $170-320 \mathrm{~nm}$ range, namely $\mathrm{CO}_{2}$, $\mathrm{SO}$, and $\mathrm{SO}_{2}$; iv) the vertical profile of the bimodal aerosols; v) the bimodal aerosol size distribution, which is defined as a log-normal distribution; and vi) the aerosol scattering functions.

We complete the analysis of the HST/STIS observations based on the RT code originally developed by Marcq et al. (2011) for continuity in comparing the HST/STIS and SPICAV observations; however, the original model is modified in two key ways, as outlined below:

- Improvements in the absorption cross-section data: We utilize the high-

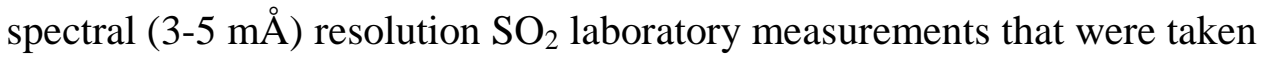


at multiple temperatures $(160 \mathrm{~K}, 198 \mathrm{~K}$, and $295 \mathrm{~K})$, by the same instrument and with near identical spectral sampling and resolution (Rufus et al. 2009, Blackie et al. 2011, Stark et al. 1999, Rufus et al. 2003) to derive the $\mathrm{SO}_{2}$ gas cross-section temperature dependence. Use of these high resolution lab measurements that fully describe the Doppler broadening of the bands minimizes the error introduced in deriving the absorption properties of the gases at the lower resolution of the observations. For the SO absorption signature, we utilize the cross-section data from Nishitani et al., (1985) and Philips et al. (1981), which are the best available.

- Improvements in the aerosol opacity model: The mode 2 extinction coefficient altitude variation is no longer represented as a step function; the new vertical profile is assumed to be a constant between 50 and $56 \mathrm{~km}$ and to decrease exponentially in the altitude range extending from $56 \mathrm{~km}$ to the user-specified upper cloud boundary (UCB) altitude (see Fig. 6). This new profile effectively thickens the upper cloud region more realistically as a function of altitude, especially at depths below $60 \mathrm{~km}$. The improved model can decrease $\mathrm{SO}_{2}$ column densities retrieved from SPICAV nadir observations by up to $50 \%$ when compared to the results published in Marcq et al. (2011).

The basic model parameters are summarized in Table III. The $\mathrm{SO}_{2}$ gas density, mode 1 aerosol opacity above the upper cloud boundary (UCB) and the $\mathrm{SO} / \mathrm{SO}_{2}$ gas density ratio are free parameters in our model that can be adjusted to match the overall continuum shape of the HST/STIS spectra and the observed gas absorption signatures due to $\mathrm{SO}_{2}$ and $\mathrm{SO}$, which are the dominant absorbers at $200-300 \mathrm{~nm}$. It is important to note that the current RT model assumes that the UCB should be equivalent to the cloud height inferred from 1.4-1.6 $\mu \mathrm{m} \mathrm{CO} \mathrm{CO}_{2}$ absorption signature (Ignatiev et al. 2009); it also assumes that mode 2 particles are only present at altitudes below the UCB. Thus, the UCB defines the upper boundary of the mode 2 particles. Additionally, the current version of the model assumes the scale heights of the mode 1 and mode 2 particles are the same. Notably, this may not be the best representation of the mode 2 particle behavior, as 
analysis of the SPICAV-IR occultation observations taken throughout the 8 year period of the Venus Express mission (Wilquet et al. 2009, Wilquet et al. 2012) indicates the larger mode 2 particles may in fact be found at altitudes greater than the previously inferred $\sim 70 \mathrm{~km}$ cloud top altitude (Esposito et al. 1983) Nevertheless, our current RT model provides the most basic representation of mode 2 particle behavior that is roughly consistent with previous observations and the current analyses of the available SPICAV and VIRTIS nadir observations and is suitable for a first order analysis of the impact of the particles on simulated spectral signatures. In particular, previous observations have shown that in the $\pm 40^{\circ}$ latitude region the cloud height relative to $\mathrm{CO}_{2}$ vertical distribution is fairly stable (Ignatiev et al. 2009). Therefore, since all the HST observations are obtained within $\pm 40^{\circ}$ latitude, we adopt a single value, $72 \mathrm{~km}$, for the UCB height when fitting all of the HST/STIS spectra. This value is consistent with the average cloud height inferred from SPICAV-IR $(1.4-1.6 \mu \mathrm{m})$ observations obtained between December 2010, and January 2011 (i.e, VEx orbits 1700 and 1750), see Table II.

Lastly, in order to accommodate any residual broadband scattering/absorption in the observations that is not straightforwardly replicated by our base model, we include a brightness scaling factor in our model. This term is equivalent to the factor by which the modeled radiance must be multiplied to match the observed albedo level at $245 \mathrm{~nm}$ (where all gas absorption, including that by $\mathrm{SO}, \mathrm{SO}_{2}$ and even $\mathrm{O}_{3}$, should be negligible). Notably, because it is the scattering behavior that determines the spectral shape at 245 $\mathrm{nm}$, the inclusion of the brightness factor term by definition implies that our fit of the mode 1 particle opacity is only an estimation and should not be considered to be absolute.

Preliminary analysis of the entire 200-300 nm spectra confirms that the longer wavelength data senses a lower altitude region than that sensed at wavelengths shortward of $260 \mathrm{~nm}$ (see Fig. 1). Thus, this paper focuses on replicating the spectral signature observed between $210-260 \mathrm{~nm}$ because i) this captures the region where the SO absorption cross-section has been measured in the laboratory, thus is the key region for determining the $\mathrm{SO} / \mathrm{SO}_{2}$ gas ratios; and $\mathrm{ii}$ ) at these short wavelengths the observed albedo is not expected to be significantly impacted by the spectral shape of the unknown UV absorber. I.e., its absorption may impact the observation, but its absorption shape is not expected to change slope rapidly with wavelength in the $210-260 \mathrm{~nm}$ region. 
Consequently, absorption due to the unknown UV absorber is not included in our simulations.

Based on the parameters described above and the other model characteristics summarized in Table III we complete a least squares fitting of the data in a two part process, first determining the likely mode 1 opacity $(\tau)$ above the cloud tops, in conjunction with the likely $\mathrm{SO}_{2}$ gas column density (based on the integration of the $\mathrm{SO}_{2}$ gas density from the top of the atmosphere (TOA) to the altitude level where optical depth unity is achieved in the total aerosol opacity); then refining our constraint of the $\mathrm{SO} / \mathrm{SO}_{2}$ ratio and corresponding $\mathrm{SO}$ gas density per latitude and time of day, by completing a second least-squares fit of the data allowing the $\mathrm{SO} / \mathrm{SO}_{2}$ gas density ratio to be a free parameter in the atmospheric model, ranging from 0-35\%. The least-squares fit is estimated relative to the expected error in each of the observed spectra, where the data error is defined based on Poisson statistics and includes the errors introduced by the sky background and the grating scattered light, as discussed in Section 3.

\begin{tabular}{|c|c|}
\hline \multicolumn{2}{|c|}{ TABLE III: Basic Model } \\
\hline free parameters & model constants \& other constraints \\
\hline & upper cloud boundary altitude (UCB): 72 km \\
\hline$\tau_{\text {mode1 }}: 0.5-3.0$ & $\mathrm{SO}_{2}$ scale height $\left(\mathrm{H}_{\mathrm{SO} 2}\right)=3.0 \mathrm{~km}$ \\
\hline $\begin{array}{l}\mathrm{SO}_{2} \text { gas density }\left(\mathrm{NSO}_{2}\right) \text { above } \\
\text { the } \tau_{\text {aerosol }}=1 \text { altitude: } 0.01-160 \mu \mathrm{m} \text {-atm }\end{array}$ & $\begin{array}{c}\tau_{\text {mode2 }} \mathrm{z}>56 \mathrm{~km} \text { exponentially decreases up to } \\
\text { UCB } \\
\tau_{\text {mode2 } 2}=\text { constant }=3.24 \text { at } 50 \mathrm{~km}<\mathrm{z}<56 \mathrm{~km}\end{array}$ \\
\hline $\mathrm{SO} / \mathrm{SO}_{2}$ gas ratio: $0-35 \%$ & $\begin{array}{l}\text { mode 1, mode } 2 \text { scale height } \\
\left(\mathrm{H}_{1}, \mathrm{H}_{2}\right)=4.28 \mathrm{~km}, 4.28 \mathrm{~km}\end{array}$ \\
\hline
\end{tabular}

We present in detail the $\mathrm{SO}_{2}$ and $\mathrm{SO}$ gas column densities and volume mixing ratios (i.e., relative to the total volume of gas, assuming a $\mathrm{CO}_{2}$ gas abundance of 0.965 ) inferred from each spatial bin as a function of latitude and time of day in Section 5.

\section{Gas Density Results: Inferred Spatial and Temporal Variability of $\mathbf{S O}$ and $\mathbf{S O}_{2}$}

The $\mathrm{SO}_{2}$ and $\mathrm{SO}$ gas column densities and mode 1 aerosol optical depths above the UCB inferred from the least-squares fitting of the data assuming $\mathrm{UCB}=72 \mathrm{~km}$ are summarized in Table IV. For all of the observations the observed spectral signatures are best fit by the model spectra for which the mode 1 optical depth encountered above the $\mathrm{UCB}$ at $245 \mathrm{~nm}$ is in the range of $\sim 1.0-2.8$. Of course, the total optical depth value at 245 
$\mathrm{nm}$ varies with altitude and is a function of all the aerosol components. Since the entire dataset is best fit when the mode 1 optical depth encountered above the UCB at $245 \mathrm{~nm}$ is $\geq 1$, the implication is that the total $245 \mathrm{~nm}$ optical depth unity altitude always occurs at an altitude greater than the UCB. In particular, with the UCB height set at $72 \mathrm{~km}$, for the fit range of mode 1 aerosol opacities, optical depth unity is always obtained within the $74.8 \pm 2.5 \mathrm{~km}$ altitude range depending on the latitude and local time of the observation. For all observations at high solar zenith angle (i.e. $\mathrm{SZA} \geq 60^{\circ}$ ) aerosol optical depth unity occurs at an altitude of $75 \pm 2 \mathrm{~km}$. Additionally, based on the aerosol profiles assumed in our model (see Section 4), above the UCB the total aerosol opacity is dependent solely on the opacity of the mode 1 particles and $\mathrm{CO}_{2}$ Rayleigh scattering.

Based on the results presented in Table IV we describe trends in the observed $\mathrm{SO}_{2}$ and SO gas distributions as well as the brightness factor as function of latitude and time of day. We discuss these trends based on both the best-fit column densities and the inferred $\mathrm{SO}_{2}$ and $\mathrm{SO}$ volume mixing ratios (VMR) where the VMRs are derived using the VIRA (International Venus Reference Atmosphere) $\mathrm{CO}_{2}$ column density (Seiff et al 1985, von Zahn and Moroz 1985) above the inferred total aerosol $\tau=1$ altitude, and assuming $0.965 \mathrm{CO}_{2}$ abundance. We also compare the HST observations to SPICAV-UV nadir observations obtained throughout the lifetime of the Venus Express mission.

In general, for the 2010 observations the dayside $\left(\mathrm{SZA}<60^{\circ}\right) \mathrm{SO}_{2}$ column densities were in the range of 2-10 $\mu \mathrm{m}$-atm, while for the 2011 observations the dayside $\mathrm{SO}_{2}$ column densities were in the range of 0.4-1.8 $\mu \mathrm{m}$-atm. The corresponding average $\mathrm{SO}_{2}$ dayside VMR on December 28, 2010, is $\sim 5$ and 10 times larger than that inferred from the January 22, 2011, and January 27, 2011 observations, respectively. The dayside SO column densities on December 28, 2010, were in the range of 0.1-0.11 $\mu \mathrm{m}$-atm thus 3.5-6x larger than the 0.08-0.20 $\mu \mathrm{m}$-atm and 0.07-0.09 $\mu \mathrm{m}$-atm ranges observed in 2011 on January 22 and January 27, respectively. More detailed analyses are provided below.

\subsection{Spatial trends in the inferred $\mathrm{SO}_{2}$ gas column density on each observation date:}

On December 28, 2010, $\mathrm{SO}_{2}$ gas column densities in the range of 2-17 $\mu \mathrm{m}$-atm were inferred from observations obtained at SZA of $25-70^{\circ}$. The inferred $\mathrm{SO}_{2}$ gas column densities also suggest a strong latitudinal variation, with the largest $\mathrm{SO}_{2}$ gas column 
densities found at the equator, at a SZA $\sim 70^{\circ}$ (Fig. 7). Notably, the gas densities detected at the equator near the terminator (i.e., at SZA $\sim 70^{\circ}$ ) are $\sim 2 \mathrm{x}$ higher than detected at the equator at a smaller SZA, implying that the $\mathrm{SO}_{2}$ gas density is also enhanced on the morning terminator. At the same time, the $\mathrm{SO}_{2}$ gas column density detected at the equator at SZA $\sim 0^{\circ}$ is almost 3 times larger than that observed near $16 \mathrm{~S}$ latitude at the same SZA, suggesting the level of enhancement near the terminator may also be latitudinally dependent.

Observations obtained on January 22, 2011 show trends similar to that observed on December 28, 2010 though the $\mathrm{SO}_{2}$ gas density range is an order of magnitude lower extending from 0.6-1.8 $\mu \mathrm{m}$-atm (Fig. 7). As seen on December 28, 2010, the inferred $\mathrm{SO}_{2}$ gas densities show strong latitudinal variation, with the peak $\mathrm{SO}_{2}$ gas density observed near equator. Likewise, the $\mathrm{SO}_{2}$ gas density observed at 27S near the terminator (SZA $70^{\circ}$ ) is $\sim 2 \mathrm{x}$ higher than observed at similar latitudes at SZA $<60^{\circ}$, suggesting that the $\mathrm{SO}_{2}$ gas density at the morning terminator is enhanced relative to the average dayside $\mathrm{SO}_{2}$ gas density. Additionally, on this date detections of the $\mathrm{SO}_{2}$ gas density near the equator just one hour prior to local noon (i.e., at $\phi \sim-15^{\circ}$ ) were made. The retrieved $\mathrm{SO}_{2}$ gas density was a $\sim$ factor of 2 lower than the values obtained $\pm 10^{\circ}$ from the equator at SZAs ranging from $20^{\circ}$ to $50^{\circ}$, i.e. between a local time of 8:40-10:40 a.m.

As discussed above, the observations obtained on January 27, 2011 were obtained with the same observing geometry as utilized on January 22, 2011. The range of $\mathrm{SO}_{2}$ gas column densities detected on January 27, 2011, is similar to that detected 5 days prior, 0.4-1.4 $\mu \mathrm{m}$-atm; however, on this date the maximum gas density is observed near $40 \mathrm{~S}$ latitude rather than at the equator [Fig. 7]. In spite of the change in the direction of the latitudinal variance between January 22 and January 27, the largest $\mathrm{SO}_{2}$ gas column densities again were observed near the terminator. In fact, for OBS4 there appears to be no other sensitivity to SZA above SZA $=40^{\circ}$ (which corresponds to detections made between $5 \mathrm{~N}$ to $25 \mathrm{~S}$ ) except for the possibility of an enhancement near the morning terminator at $\mathrm{SZA}=70^{\circ}$ between $25 \mathrm{~S}$ and 36S latitude. However, comparison of the gas densities detected during OBS4 and OBS5 at comparable latitudes at both high and low SZAs does not show strong evidence of enhancement in the observed gas density detected at high SZA relative to that the gas density detected at SZA $<60^{\circ}$. This suggests 
that the increased gas density evident near the terminator in both OBS4 and OBS5 is driven entirely by the mechanism supporting the observed latitudinal gas density gradient.

\subsection{Trends in the inferred SO gas column density per observation date:}

The observed SO gas column densities display behavior similar to the observed $\mathrm{SO}_{2}$ gas column densities: the largest values are observed at the terminator on all three days [Fig. 9], near the equator on December 28 and January 22 [Fig. 8], and at southerly mid-latitudes on January 27. Additionally, the sample correlation coefficient between the $\mathrm{SO}_{2}$ and $\mathrm{SO}$ gas column densities (noted as $\mathrm{NsO}_{2}$ and $\mathrm{Nso}$, respectively) across all three observing dates is 0.97, ignoring uncertainties. Based on a linear regression weighted by the uncertainties on the $\mathrm{SO}$ gas column densities but ignoring the uncertainties on the $\mathrm{SO}_{2}$ gas column densities, $\mathrm{Nso}=(0.08 \pm 0.02) * \mathrm{NsO}_{2}+(0.04 \pm 0.02)$. Within the uncertainties on the retrievals, the inferred SO gas column densities are basically invariant between $\pm 20^{\circ}$. On December 28, 2010 the inferred SO gas density range is slightly lower in the $25 \mathrm{~N}-40 \mathrm{~N}$ latitude range than in the $\pm 20^{\circ}$ equatorial band; while on Jan 27 , the inferred SO gas density range is slightly higher in the 20S-40S latitude range than in the $\pm 20^{\circ}$ equatorial band. This would seem to imply that variability in the SO gas density is primarily latitudinally driven, and that the gradient in the latitude dependence shows opposing trends in the northern and southern hemispheres. However, it is more likely that the difference in the northern and southern latitude gradients is a function of temporal variation (as a reversal in the $\mathrm{SO}_{2}$ latitude gradient between these dates is also evident), rather than a standing opposing trend in the latitude gradient of the SO gas density between hemispheres (see discussion below). Repeated acquisition of spatially resolved observations that can uniquely measure both the $\mathrm{SO}_{2}$ and $\mathrm{SO}$ gas densities would help to clarify this point.

\subsection{Comparisons among observation dates:}

On all three dates the $\mathrm{SO}_{2}$ gas density detected in the equatorial region between \pm $8^{\circ}$ latitude was strongly overlapping, thus implying limited sensitivity in the equatorial 
$\mathrm{SO}_{2}$ gas density to SZA, for SZA $<60^{\circ}$; and on January 27 , the latitudinal extent over which the observed equatorial gas density was strongly overlapping was broader corresponding to $\pm 15^{\circ}$ latitude [Fig. 7]. The only exception to the inferred equatorial insensitivity to $\mathrm{SZA}$, for $\mathrm{SZA}<60^{\circ}$ is the factor of 2 difference between the $\mathrm{SO}_{2}$ gas density observed at $\sim 2 \mathrm{~N}$, at $\mathrm{SZA}=17^{\circ}$ vs. that observed between $\pm 10^{\circ}$ at SZAs of $20-50^{\circ}$ on January 22, 2011. Comparison of the data acquired at a single latitude at both SZA $\geq$ $65^{\circ}$ and SZA $<60^{\circ}$ indicates that on both December 28, 2010 and January 22, 2011 the $\mathrm{SO}_{2}$ gas column density near the terminator is a factor of 1.8 to 2.4 larger than that observed at a smaller SZA. On January 27, 2011 comparison of the gas density retrievals made at near equivalent latitude but varying SZA, indicates that the observed gas densities are equivalent within the uncertainty of the fits (see Table IV). This behavior is different from that observed on the other two dates. Thus, as pointed out above, though the gas density observed at $36 \mathrm{~S}$ at $\mathrm{SZA}=66^{\circ}$ is $1.4 \mathrm{x}$ greater than that observed at $31 \mathrm{~S}$ at $\mathrm{SZA}=60^{\circ}$, this increase is likely to be either purely latitudinally driven or just a localized enhancement, but it is not consistent with changes driven by the increased SZA [Fig. 7].

As discussed above, the $\mathrm{SO}_{2}$ gas column density inferred from each observation is strongly latitudinally dependent; however, the latitudinal gradient changes sign. Because the January 22, 2011, and January 27, 2011, observations have the same observing geometry and are separated by one cloud top rotation period, the reversal of the latitudinal gradient from that observed on the other two dates is especially significant, suggesting that something other than (or in competition with) a stable pattern in time of day and latitude impacts the observed $\mathrm{SO}_{2}$ gas densities. Looking more closely at the inferred atmospheric properties, the $\mathrm{SO}_{2}$ gas column densities observed between $\pm 10^{\circ}$ latitude on January 27, 2011, are 2-3 times smaller than were observed in that region on January 22, 2011, and the brightness factors fit to the spectra obtained at these latitudes on January 27 are in the range of 0.7-0.73, also smaller than the 0.74-0.83 values inferred from the January 22 spectra obtained within this same latitudinal range. On the other hand, while the near-terminator $\left(\mathrm{SZA} \geq 60^{\circ}\right) \mathrm{SO}_{2}$ gas densities observed in January 2011 were 10-20x lower than the near-terminator values detected on December 28, 2010, the values detected on January 22 and January 27, 2011 were similar corresponding to 0.81.4 and $0.4-1.4 \mu \mathrm{m}$-atm, respectively. 
The decrease in the equatorial $\mathrm{SO}_{2}$, $\mathrm{SO}$ gas density and dayside albedo brightness inferred from the comparison of the December 2010 and January 2011 HST observations may be indication of a number of physical scenarios. E.g., the darkening observed in the 2011 observations may be an indication of the transit of the well-known Y-feature in and out of the slit between late December 2010 and late January 2011, which in itself is an indicator of variance in the density of the unknown UV absorber at these latitudes, due to zonal winds(=advection). However, this is not likely. For one, if the decreased albedo were due to increased presence of the unknown UV absorber, the implication would be that the $\mathrm{SO}_{2}$ gas was decreasing as the unknown UV absorber was increasing which is opposite to previously observed trends (Esposito, 1980; Esposito and Travis, 1982). Additionally, although the VMC imaging is limited to the southern low latitude regions, and our ability to readily identify the contrast in the images is significantly compromised at the latitudes observed closest to the edge of the Venus' observable disk (see Fig. 5), based on the available imaging there is no evidence of the presence of the Y-feature in the regions coincidently observed by HST and VMC. And lastly, while it is possible the Y-feature was present at low northern latitudes outside of the field of view of VMC, this would require that the narrowest region of the feature intersected the HST slit, since the feature is known to be symmetrical and is expected to extend $\pm 20^{\circ}$ of the equator in most cases (Rossow et al. 1980)

A far more plausible interpretation of the coincident decrease in the $\mathrm{SO}_{2}$ gas density and the albedo brightness in January 2011 is that density of the (UV bright) submicron haze particles in January 2011 was lower than that present at the cloud tops in December 2010 due to a decrease in upwelling of fresh aerosols or some other dynamically driven change in the chemical processing/formation of the aerosols. A decrease in the mass density of the sub-micron haze particles within the observed time period is consistent with the expected correlation (and co-location) of sulfur-bearing aerosols and the density of the $\mathrm{SO}_{2}$ gas. Additionally, a decrease in the key absorbers (such as micron sized aerosols and other gases) responsible for the infamous dark markings in Venus' cloud tops should result in cloud top brightness at UV wavelengths that is fairly uniform. Interestingly, the cloud region coincidently observed by HST and VMC shows limited contrast, the general the lack of strong contrast features may be 
partly due to the viewing angle. However, inspection of the afternoon quadrant of the projected December 27, 2010 VMC images shows distinct patterns in the cloud contrast at latitudes closest to the edge of the VMC field of view — so that we can confidently say that if there had been significant contrast in the low-latitude morning quadrant cloud top region due to the presence of dark absorption patches it would have been observable [Fig. 10]. The observation of uniformly bright clouds, free of dark absorption patches is consistent with an atmosphere in which vertical mixing and a cut-off of the supply of UV absorbers (including but not limited to $\mathrm{SO}_{2}$ gas) from below the cloud deck has been depressed due to the cooling of the atmosphere (see Titov et al. 2008).

Additionally, while the overall contrast of the $0.36 \mu \mathrm{m}$ January 22 and January 27, 2011 VMC images was uniform, comparison of the calibrated flux intensity of the cloud tops directly beneath the HST/STIS slit field of view indicates that the $0.36 \mu \mathrm{m}$ brightness of the clouds northward of 35S was 15\% darker on January 27 than on January 22 [Fig. 11]. The gradient of the cloud brightness was also observed to change from increasing towards the equator at all latitudes, to becoming near constant northward of $20 \mathrm{~S}$ latitude between the two dates. At the same time, the $\mathrm{SO}_{2}$ gas density decrease observed between those two dates was most prominently evident northward of 15Sleading to a change in the gradient of the $\mathrm{SO}_{2}$ latitudinal variation. Therefore, the latitude where the most significant change in the cloud brightness was observed coincides with the latitudes where the most significant change in the $\mathrm{SO}_{2}$ gas density was observed.

\subsection{Comparisons to SPICAV-UV observations:}

Due to thermal constraints, SPICAV-UV nadir observations could not be included in the original VEx-VCO coordination plan; therefore, no SPICAV-UV nadir observations were taken on the same date as the HST observations. However, SPICAVUV nadir observations were obtained within 1-2 days of the December 2010, HST observations, and again in March 2011, 1 month subsequent to late January 2011 HST observations. Comparison of the $\mathrm{SO}_{2}$ column densities and overall cloud top brightness levels inferred from the HST and SPICAV data obtained during these time periods indicates that the overall atmospheric variability inferred from the two observation platforms is the same. For example, the average December $2010 \mathrm{SO}_{2}$ column density 
retrieved by SPICAV was $\sim 17 \pm 4 \mu \mathrm{m}$-atm; for March 2011 the value was $\sim 0.7 \pm-0.4 \mu \mathrm{m}$ atm. In both cases the order of magnitude of the SPICAV-nadir $\mathrm{SO}_{2}$ gas density retrievals was comparable to 2-12 $\mu \mathrm{m}$ and 0.4-1.9 $\mu \mathrm{m}$ values observed by HST in late December 2010 and late January 2011, respectively. Notably the December 2010 SPICAV values were slightly greater than the December 2010 HST retrievals, but this is likely because those observations were obtained at low-latitudes on the afternoon rather than on the morning quadrant. Additionally, both the HST and the nadir SPICAV-UV datasets indicate a decrease in the $\sim 0.24 \mu \mathrm{m}$ brightness of Venus' low-latitude dayside (i.e. for SZA $<60^{\circ}$ ) cloud tops between Dec 2010 and March 2011 [Fig. 12]. Additionally, the acquired low-latitude ( $<40^{\circ}$ latitude) dayside HST observations capture the high and low range of the low-latitude $\mathrm{SO}_{2}$ gas column densities observed by SPICAV-UV at lowlatitudes throughout the entirety of 2011. In fact, the average dayside VMR values derived from the HST observations was in the range of $43 \pm 37 \mathrm{ppb}$ between December 2010 and February 2011, and is identical to the average VMR derived from all the SPICAV observations obtained during 2011 [Fig. 2].

Because of the lower spectral resolution of the SPICAV-UV nadir observations the SO gas column density was not directly retrieved from the available SPICAV-UV observations. Therefore, we do not make a one to one comparison of the HST and SPICAV SO gas density retrievals. Instead, we point out that while Marcq et al (2011) simply assumed the SO gas column density was consistently equivalent to $10 \%$ of the retrieved $\mathrm{SO}_{2}$ gas column density, the HST retrieved $\mathrm{SO} / \mathrm{SO}_{2}$ ratio ranged $\sim 7-18 \%$. These results imply that the order of magnitude of the SPICAV derived SO column densities are reasonable. However, the results also emphasize that in the absence of adequate spectral resolution the latitudinal and local time variation of the $\mathrm{SO} / \mathrm{SO}_{2}$ ratio, which may be indicative of changes in the vertical mixing of the atmosphere with time and latitude, cannot be tracked or measured.

Both the larger SPICAV-UV dataset and our small sampling of HST observations indicate that instabilities in the $\mathrm{SO}_{2}$ latitude gradient can be observed [Fig. 13]. The longterm average behavior recorded in the SPICAV-UV nadir observations indicates a decrease in the $\mathrm{SO}_{2}$ gas column density with increasing latitude is the "normal state" of the latitudinal variation, but the reversed $\mathrm{SO}_{2}$ latitude gradient trend is evident when the 
lowest equatorial $\mathrm{SO}_{2}$ gas column density is detected (Marcq et al. 2013). Surprisingly, similar to the trends seen in the long-term SPICAV-UV data, our analysis indicates that on January 27, 2011 when the lowest equatorial $\mathrm{SO}_{2}$ gas column density was detected the latitudinal gradient is reversed from the "normal" gradient that increases with decreasing latitude to a gradient that increases as the latitude increases. I.e., on January 27, 2011 the $\mathrm{SO}_{2}$ gas column densities detected by HST within $10^{\circ}$ of the equator were equal to $0.4 \pm 0.2 \mu \mathrm{m}$-atm, which is $\sim 2.5$ - $25 \mathrm{x}$ lower than the $1.0 \pm 0.2$ and $10.0 \pm 2.0 \mu \mathrm{m}$-atm values observed by HST at equivalent latitudes on January 22, 2011 and December 28, 2010, respectively, at SZAs $<60^{\circ}$.

Interestingly, Marcq et al. (2013) suggests that the gradient of the $\mathrm{SO}_{2}$ latitudinal variation at low latitudes is dependent on the supply of $\mathrm{SO}_{2}$ from below the cloud deck at low latitude. In particular, the Marcq et al. (2013) model specifically proposes that when the vertical mixing rates in the atmosphere are suppressed and remain suppressed for a 45 day time period the latitudinal gradient will reverse so that $\mathrm{SO}_{2}$ increases away from the equator; however, the gas density at latitudes outside of the ascending node of the Hadley cell will remain fairly stable over the 4-5 day period, since the photolysis rates at these higher latitudes are also lower. Thus, the observance of low gas densities at the cloud tops in January 2011 by HST, and the corresponding change in sign of the latitudinal gradient in the HST observations from January 22, 2011, to January 27, 2011 at low latitudes, are consistent with the predictions of the Marcq et al. (2013) model.

Additionally, although there was a $2.5 \mathrm{x}$ decrease in the $\mathrm{SO}_{2}$ gas density retrieved from the equatorial January 22 and January 27, 2011 observations, the $\mathrm{SO}_{2}$ gas density retrievals obtained between 25-36S latitude in the near-terminator $\left(\mathrm{SZA} \geq 60^{\circ}\right.$ ) region are equivalent. At a minimum, this implies that whatever the process is that led to the change in the sign of the latitudinal $\mathrm{SO}_{2}$ gradient between the two dates, its impact is most strongly evidenced at low SZA where the impact of photochemical processing is also greatest. Notably, the basic tenet of the Marcq et al. model is that the gas density at high latitude remains stable because of limited photochemical processing in the higher latitude regions. Since this argument also holds for high SZA observations, the observed stability in the near-terminator $\mathrm{SO}_{2}$ gas density retrievals obtained on January 22 and January 27, 2011 is also consistent with the basic tenets of the Marcq et al. model. 
If we assume that the observed $0.36 \mu \mathrm{m}$ cloud top brightness maps to changes in the $\mathrm{SO}_{2}$ and $\mathrm{H}_{2} \mathrm{SO}_{4}$ aerosol abundance then the fact that the observed VMC cloud brightness is invariant poleward of 35S latitude on January, 22 and January 27, 2011 while clear differences in the brightness gradient are evident north of $20 \mathrm{~S}$ latitude is also an indication that the latitudinal variability in the cloud top brightness is directly linked to changes in the upward pumping of both the $\mathrm{SO}_{2}$ gas and other collocated aerosols near the equator, with limited change in the downward flux and photochemical processing of the gas and aerosols at higher latitudes; as also predicted by the Marcq et al. (2013) model.

As discussed above, both the HST and SPICAV-UV nadir observations show that the spectral brightness near $0.24 \mu \mathrm{m}$ became darker in the time period from late December 2010 to late January 2011; additionally, the $0.24 \mu \mathrm{m}$ planet brightness was observed by SPICAV to darken over the lifetime of the VEx mission between 2007 and 2011 [Fig. 12]. The darkening observed by HST at $0.245 \pm 0.004 \mu \mathrm{m}$ in spite of the lack of observable dark patches at $0.36 \mu \mathrm{m}$ highlights the fact that the $0.245 \pm 0.004 \mu \mathrm{m}$ albedo levels track the haze brightness, and is indicative of the haze properties. In particular, the higher the density of the sub-micron particles the brighter the haze, and conversely the lower the density of the sub-micron particles the more transparent the haze, leading to overall darkening at the cloud tops. Thus, the darkening of the $0.245 \pm 0.004 \mu \mathrm{m}$ abledo levels observed by HST suggests that the sub-micron particle density decreased (either due to a lower influx or due to the coalescence of the particles into larger particles) in the time period from late December 2010 to late January 2011. The fact that SPICAV also observed an overall decline in the $0.24 \mu \mathrm{m}$ brightness of the planet may also suggest that there was an overall decrease in the sub-micron density over the lifetime of the VEx mission between 2007 and 2011.

\section{Gas Density Behavior Inferred from Contemporaneous Gas Density Measurements}

\subsection{Insights from the coordinated VEx/SOIR +HST/STIS UV observations:}

As outlined in Section 2, the HST/STIS observations were coordinated with planned VEx/SOIR observations obtained on December 28, 2010, on the morning 
terminator at $31 \mathrm{~S}$ latitude. The goal of the coordinated HST/STIS+VEx/SOIR observations was to obtain data on $\mathrm{SO}_{2}$ and $\mathrm{SO}$, near the upper cloud top where photolysis is one of the primary drivers of the sulfur chemistry cycle. SOIR's solar occultation observations can provide the $\mathrm{SO}_{2}$ abundance profile along the terminator at 65-100 km with vertical resolution ranges that vary from 0.2-0.7 km at high northern latitudes, to 2.0-7.0 km in the Southern hemisphere (Mahieux et al 2010). On December 28, 2010, the SOIR vertical resolution was $\sim 7 \mathrm{~km}$. On this date, SOIR was only able to positively detect $\mathrm{SO}_{2}$ gas (i.e. set more than an upper limit to its number density) at the terminator at altitudes of $77 \pm 3.5 \mathrm{~km} \& 78 \pm 3.5 \mathrm{~km}$ (6.64-5.39 mbar) above the surface; the retrieved $\mathrm{SO}_{2}$ number density was $\sim 5.3 \pm 0.2 \times 10^{10} \mathrm{~cm}^{-3}$ at each of these altitudes.

Notably, both the $\mathrm{SO}_{2}$ and $\mathrm{CO}_{2}$ absorption signatures are recorded in the SOIR observations, allowing for the retrieval of the vertical density profiles of both gases, as well as the precise definition of the $\mathrm{SO}_{2} / \mathrm{CO}_{2}$ mixing ratio at each altitude where the $\mathrm{SO}_{2}$ gas was positively detected, and the corresponding $\mathrm{SO}_{2} \mathrm{VMR}$ (assuming $\mathrm{CO}_{2}$ abundance of 0.965). On December 28, 2010, SOIR retrieved the $\mathrm{CO}_{2}$ vertical number density profile at high precision in the $90-113 \mathrm{~km}$ altitude range, below this altitude the $\mathrm{CO}_{2}$ number density values are extrapolated based on the behavior observed in the 90-113 km region and a climatological $\mathrm{CO}_{2}$ profile for lower altitudes (Mahieux et al. 2014a). The extrapolated $\mathrm{CO}_{2}$ number density values are equal to $2.1 \times 10^{17} \mathrm{~cm}^{-3}$ and $1.8 \times 10^{17} \mathrm{~cm}^{-3}$ at $77 \pm 3.5 \mathrm{~km}$ and $78 \pm 3.5 \mathrm{~km}$, respectively; thus, implying that the corresponding $\mathrm{SO}_{2}$ abundance (VMR) was equal to 290 and $240 \mathrm{ppb}$ in the two respective altitude bins. Notably, the average $\mathrm{CO}_{2}$ number density inferred from SOIR between 74 and $81 \mathrm{~km}$ was $\sim 1.9 \times 10^{17} \mathrm{~cm}^{-3}$, while the value derived from VIRA1 is equal to $\sim 2.6 \times 10^{17} \mathrm{~cm}^{-3}$. In fact, we find that the $\mathrm{CO}_{2}$ number densities derived from the SOIR observations on December 28,2010 , are consistently $\sim 1.37 \pm 0.03 x$ smaller than the values cataloged in VIRA at each altitude in the 74-81 km range.

In order to remove any confusion regarding the consistency of the HST and SOIR retrievals we first isolate the average $\mathrm{SO}_{2}$ number density expected in the 74-81 km range from the HST data analysis, and then utilize the same value for the average $\mathrm{CO}_{2}$ number density expected between 74-81 km as inferred from the SOIR observations to derive the $\mathrm{SO}_{2} / \mathrm{CO}_{2}$ mixing ratios. In particular, in our model, in order to define specific $\mathrm{SO}_{2}$ 
column density values at the altitude where optical depth unity is obtained in the aerosol profile the $\mathrm{SO}_{2}$ number density vertical profile is defined as a function of the $\mathrm{SO}_{2} / \mathrm{CO}_{2}$ mixing ratio, assuming that both the bulk $\mathrm{CO}_{2}$ atmosphere and $\mathrm{SO}_{2} / \mathrm{CO}_{2}$ mixing ratios are exponentially decreasing between $60-100 \mathrm{~km}$. We utilize the $\mathrm{SO}_{2}$ number density profile inferred from the coincident HST observations obtained near the terminator $\left(\mathrm{SZA}=70^{\circ}\right.$ ) at 16S latitude for the comparison to the SOIR observations, since this gas density retrieval was obtained within $\sim 15^{\circ}$ of the SOIR impact point at 31 S latitude, and given the slow variation of the spectral signatures with latitude it is likely to be representative of the gas density located at $16 \pm 3 S$. In this case we find that the average number density observed at $16 \pm 3 S$ latitude near the terminator would have been equal to $5.4 \times 10^{10}$ between 74-81 km; thus, coinciding with the values retrieved from the SOIR observations (Fig. 14) and implying that near identical $\mathrm{SO}_{2} / \mathrm{CO}_{2}$ mixing ratios will be retrieved, if identical $\mathrm{CO}_{2}$ profiles are utilized.

Although coincident HST and SOIR observations were obtained only on December 28, 2010, the $\mathrm{SO}_{2}$ number densities and $\mathrm{SO}_{2} / \mathrm{CO}_{2}$ ratios inferred from the remaining HST observations in the 74-81 km range can be compared to the coincident 2010 SOIR observations (see Fig. 14) as well as the average behavior observed throughout the lifetime of the mission (see Table V). The temporal variation in the HSTinferred near-terminator ( $\mathrm{SZA}=70^{\circ}$ ) $\mathrm{SO}_{2}$ number density maps directly to the nearterminator $\mathrm{SO}_{2}$ column density variation discussed in Section 5; thus, the average 74-81 $\mathrm{km} \mathrm{SO} 2$ number density values inferred from the HST observations are respectively a factor of 5 and 10 lower on January 22 and 27, 2011 than observed by SOIR and HST on December 28, 2010 (Fig. 14). The respective $\mathrm{SO}_{2} / \mathrm{CO}_{2}$ ratios for these dates are 280, 52 and $25 \mathrm{ppb}$ if the $\mathrm{CO}_{2}$ number density value inferred from the SOIR observations obtained at 74-81 km on December 28, 2010 is utilized, implying $\mathrm{SO}_{2}$ VMRs of 290, 54 and $26 \mathrm{ppb}$ on these dates (note: the mixing ratio values would be a factor of 1.4 smaller if the VIRA $\mathrm{CO}_{2}$ profiles are assumed). The inferred December 28, $2010 \mathrm{SO}_{2}$ mixing ratio corresponds to the highest values derived from the low-latitude (i.e. $\leq 40^{\circ}$ from the equator) 2006-2013 SOIR observations obtained between 75-80 km (Mahieux et al. 2014b), while the values inferred from the HST observations of Venus' morning 
terminator in late January 2011 are consistent with the lowest low-latitude $\mathrm{SO}_{2} \mathrm{VMR}$ values retrieved by SOIR throughout the VEx mission (see Table V).

\subsection{Insights from the coordinated HST/STIS UV + sub-mm JCMT observations:}

As an additional complement to the coordinated HST and VEx observations, ground-based 346.65217 and $346.52848 \mathrm{GHz}$ observations that are sensitive to the respective $\mathrm{SO}_{2}$ and $\mathrm{SO}$ gas abundances in the 70-100 km region of Venus' atmosphere were obtained between December 28, 2010, and January 30, 2011, using the James Clerk Maxwell Telescope (JCMT) on the Mauna Kea summit in Hawaii (see Table II). On these dates Venus was observed at phase angles of $97-78^{\circ}$ (phase $0.44-0.60$ ). The JCMT observations integrate over a 14 " diameter beam. Thus, on each date the JCMT beam was placed at 2 positions offset from the center of Venus' 28-20" such that one beam isolated and observed only the dayside morning quadrant (local Venus time 06:00-12:00), and the other beam isolated and observed only the nightside quadrant (local Venus time 00:00-06:00). We focus only on the JCMT observations obtained on the morning quadrant, since the $0.2-0.3 \mu \mathrm{m}$ HST data is only sensitive to the dayside solar reflected signature and on these dates only the dayside morning quadrant was observable from the Earth (see Fig. 4).

To properly interpret the significance of the coincident JCMT and HST retrievals it is important that the observing and analysis parameters of the JCMT data are well understood. For one, since the JCMT observations are beam-integrated they provide a measure of the average dayside morning quadrant gas absorption signature for each date of observation. Additionally, because of the orientation of the JCMT beam, the terminator is located along the edge of the arc of the observing beam; thus, the contribution that this foreshortened region makes to the observed $\mathrm{SO}_{2}$ and $\mathrm{SO}$ gas absorption signatures is negligible. Consequently, it is best to compare the JCMT results to the average dayside behavior inferred from the spatially resolved HST observations at SZA $<60^{\circ}$. We also point out that the gas absorption signatures recorded in the JCMT observations uniquely characterize the average atmospheric pressure at which the gas detections were made. JCMT observations are converted from pressure to altitude coordinates using a temperature profile specified on a $2 \mathrm{~km}$ grid from 66 to $120 \mathrm{~km}$, 
representative of the average of the spatially averaged dayside temperature measured with the JCMT in the period 1999-2002 (see Clancy et al. 2003). Like $\mathrm{SO}_{2}$ abundance, those temperatures are fundamentally measured as a function of pressure, not altitude. The methods used to assign altitude to the pressure and temperature profiles are described in detail in Clancy et al. (2012), but in brief rely on the assumption of hydrostatic equilibrium and the self-calibration of the derived temperature and gas abundance profiles based upon RT modeling of lower atmospheric continuum emission to define a radiative boundary at $65 \mathrm{~km}$.

We also emphasize that the spectral shape observed in the sub-mm is sensitive to both the magnitude and the shape of the assumed vertical VMR profile of $\mathrm{SO}$ and $\mathrm{SO}_{2}$ throughout the 70-100 $\mathrm{km}$ region. Thus, the sub-mm observations can be used to distinguish a low-order power-law (or slower) increase with increasing altitude in the $\mathrm{SO}_{2}$ and SO mixing ratios from an exponential (or faster) increase within that altitude range (figures 6-8 of Sandor et al. 2010). In general, all previously obtained sub-mm observations of Venus' atmosphere are well fit by a simple 2 layer model, where the abundance (VMR) above a defined cut-off altitude is significantly higher than below that altitude (Sandor et al. 2010, Encrenaz et al. 2014). If the VMR profile below the cut-off altitude decreases exponentially or faster with increasing altitude then the magnitude of the inferred VMR below the cut-off altitude cannot be distinguished from zero. Thus far, all JCMT obtained high S/N sub-mm observations of Venus' atmosphere indicate that, taking into consideration the quoted altitude uncertainty per observation, the cut-off altitude between the two layers is located somewhere between $85 \pm 3 \mathrm{~km}$. Analysis of these previously obtained high $\mathrm{S} / \mathrm{N}$ observations of Venus' atmosphere also indicates that the observed $\mathrm{SO}_{2}$ and $\mathrm{SO}$ absorption band shape observations are compatible with vertical profiles for the $\mathrm{SO}_{2}$ and $\mathrm{SO}$ mixing ratios that are constant or weakly linear above $85 \pm 3 \mathrm{~km}$, but are inconsistent with an exponential increase in the abundances at those altitudes. Additionally, below $85 \pm 3 \mathrm{~km}$ the $\mathrm{SO}_{2}$ and $\mathrm{SO}$ VMR profiles must decrease exponentially (or faster) with increasing altitude. Although the S/N of the JCMT observations coincidentally obtained with HST was poor, the shape of the beamintegrated sub-mm gas absorption band detections obtained by JCMT coincidently with the HST/STIS observations is similar to previous JCMT observations. Consequently, the 
relative $\mathrm{SO}_{2}$ and $\mathrm{SO}$ gas abundance values and vertical distributions retrieved from these observations follow the same two-layer atmospheric model description given above (Sandor, pers. communication), where for this dataset a constant VMR is defined between 86 and $100 \mathrm{~km}$, and the cut-off altitude of the 2-layer model is $84 \pm 2 \mathrm{~km}$.

As discussed above, analysis of the HST observations retrieves the $\mathrm{SO}_{2}$ and $\mathrm{SO}$ gas column densities expected at the altitude where optical depth unity is reached in the model aerosol profile (which on average is between $75 \pm 2 \mathrm{~km}$ ). The column density of each species at a given altitude is defined as a function of the respective, $\mathrm{SO}_{2} / \mathrm{CO}_{2}$ and $\mathrm{SO} / \mathrm{CO}_{2}$ mixing ratios, which are assumed to be exponentially decreasing with altitude. As a result, the $\mathrm{SO}_{2} / \mathrm{CO}_{2}$ and $\mathrm{SO} / \mathrm{CO}_{2}$ mixing ratio values obtained by simply averaging the $\mathrm{SO}, \mathrm{SO}_{2}$ and $\mathrm{CO}_{2}$ number density profiles between 75 and $85 \mathrm{~km}$ is equivalent to the value derived by integrating the $\mathrm{SO}, \mathrm{SO}_{2}$ and $\mathrm{CO}_{2}$ column densities between 110-75. Given the comparability of the SOIR and HST results between 74 and $81 \mathrm{~km}$, we are confident that the assumption of an exponentially decreasing $\mathrm{SO}_{\mathrm{x}} / \mathrm{CO}_{2}$ mixing ratio at 74$81 \mathrm{~km}$ is reasonable, and we are confident that $\mathrm{SO}_{\mathrm{x}} / \mathrm{CO}_{2}$ mixing ratios derived from the HST analysis uniquely define the average $\mathrm{SO}_{\mathrm{x}}$ abundances in that altitude range.

Although the $\mathrm{SO}_{2}$ and SO VMR values are retrieved from sub-mm gas absorption signatures that are averaged between latitudes extending from $\pm 90^{\circ}$ from the equator, the retrieved values are biased by whichever latitudinal regions have the highest $\mathrm{SO}_{2}$ and $\mathrm{SO}$ gas densities. As discussed above, the analysis of the JCMT sub-mm observations provides a unique retrieval of the magnitude of the average dayside $\mathrm{SO}_{2}$ and $\mathrm{SO} \mathrm{VMR}$ values and their vertical distributions in the $86-100 \mathrm{~km}$ altitude range. Contrastingly the HST observations uniquely identify the $\mathrm{SO}_{\mathrm{x}}$ column density above specific latitude and longitude coordinates. The latitudinal $\mathrm{SO}_{2}$ gradients evident in the HST observations suggest that on December 28, 2010 and January 22, 2011 the gas signatures were most prominent at low-latitude. If we assume that the latitudinal gas distributions observed in the lower 74-81 $\mathrm{km}$ region are propagated to the higher mesospheric and lower thermospheric regions sampled by the JCMT data (see Fig. 1) then it is implied that the disk-averaged sub-mm JCMT observations obtained contemporaneous to those dates were also dominated by the low-latitude gas density behavior. 
Thus, the combined HST and JCMT results provide information on the average dayside behavior at low-latitudes through most of Venus' upper mesosphere and within a $10 \mathrm{~km}$ portion of Venus' thermosphere for each date of observation. While the profiles obtained between January 28 and 30, 2011 may in fact characterize the upper mesospheric behavior prominent when the $\mathrm{SO}_{2}$ gas density is dominantly located in Venus' mid-latitude regions. This information is highly desirable for better understanding of photochemical processing within Venus' low-latitude mesosphere. For example, the combined HST and JCMT average dayside vertical profile data confirms the altitude dependence inferred from recent photochemical models (Krasnopolsky 2012, Mills 1998, Mills and Allen 2007, Petrass 2013, Yung et al. 2009) that predict that the $\mathrm{SO}_{2} \mathrm{VMR}$ profile is near constant above $\sim 75 \pm 10 \mathrm{~km}$, and exponentially decreasing below $75 \pm 10$ $\mathrm{km}$, assuming that the sulfur chemical processing rates are consistent with that observed on the Earth. At the same time, Zhang et al. (2012) and Petrass (2013) show that a small inversion layer (Fig. 15) may exist in the altitude range segregating the upper and lower mesosphere if the sulfur chemical processing rates are significantly altered from the rates observed on Earth. The observational results presented here do not preclude the existence of such an inversion layer in the $\sim 81$ to $86 \mathrm{~km}$ altitude range that spans the gap between the HST retrieval altitudes and the altitude range wherein non-zero $\mathrm{SO}_{2}$ and $\mathrm{SO}$ VMR values are uniquely retrieved from the 2-layer model fit of the coincident JCMT sub-mm data.

Although the uncertainties on the JCMT retrievals are unusually large due to poor observing conditions (Sandor, pers. communication), we compare the magnitude of the mesospheric $\mathrm{SO}_{\mathrm{x}}$ abundances inferred from the JCMT (Sandor, pers. communication) and HST data retrievals in Figure 16. These comparisons indicate that at altitudes $\geq 86 \mathrm{~km}$ the magnitude of the dayside average upper mesospheric SO VMRs are larger than the average values inferred for the 74-81 km region from the HST observations, although the mutual uncertainties nearly overlap [Fig. 16]. JCMT's higher SO VMR retrieval values are expected based on the 2 layer model behavior retrieved from the analysis of the JCMT observations - which indicates that the $\mathrm{SO}_{\mathrm{x}}$ species vertical distribution between 86 and $100 \mathrm{~km}$ is constant and that the absolute magnitude of the abundance is >> than that observed at altitudes $\leq 84 \mathrm{~km}$. 
On the other hand, the dayside average $\mathrm{SO}_{2}$ VMR values inferred from the two datasets [Fig. 16] are similar and show similar temporal variability. The similarity in the absolute abundance values inferred from the two upper mesospheric altitude regions is surprising, but still does not contradict the JCMT 2-layer model. This is because the HST values are derived from an exponentially decreasing $\mathrm{SO}_{2} / \mathrm{CO}_{2}$ vertical profile, thus the retrieved values meet the requirement of the JCMT fits that the profile below $84 \pm 2 \mathrm{~km}$ decreases exponentially with altitude. Additionally, given that the upper mesosphere vertical profile inferred from the JCMT data is constant with altitude, the integration of the JCMT derived $\mathrm{SO}_{2}$ VMR abundance values at altitudes $\geq 86 \mathrm{~km}$ results in a total integrated $\mathrm{SO}_{2} \mathrm{VMR}$ that is $>>$ than the value derived from integrating between 74 and $81 \mathrm{~km}$ the exponentially decreasing $\mathrm{SO}_{2} / \mathrm{CO}_{2}$ vertical profiles used in the fitting of the HST data. This fact also confirms the basic properties of the 2-layer atmosphere model used to fit the JCMT observations.

For completeness we point out that the $\mathrm{CO}_{2}$ densities inferred from the SOIR observations imply that the HST retrieved $\mathrm{SO}_{\mathrm{x}}$ abundances are likely $1.4 \mathrm{x}$ higher than listed in Table IV (plotted in Fig. 16). However, application of the 1.4x factor does not change significantly the relative differences/similarities between the magnitude of the inferred JCMT and HST $\mathrm{SO}_{2}$ and SO VMR values.

In terms of the $\mathrm{SO}_{\mathrm{x}}$ ratios, the $74-81 \mathrm{~km}$ disk-average dayside $\mathrm{SO}_{2} / \mathrm{SO}$ ratio derived from the HST observations is in the range of $4-25$, which is $1.3-8 \mathrm{x}$ greater than the 1-3 disk-averaged $\mathrm{SO}_{2} / \mathrm{SO}$ ratio inferred from the dayside JCMT observations obtained in the same time period for altitudes $\geq 86 \mathrm{~km}$. The decrease in the dayside $\mathrm{SO}_{2} / \mathrm{SO}$ gas abundance ratio with altitude is expected, and is consistent with photochemical models presented in Sandor et al. 2010, Zhang et al. 2012, Krasnopolsky 2012 that assume that $\mathrm{SO}_{2}$ and $\mathrm{SO}$ are in photochemical equilibrium with each other [Fig. 17]. Thus, according to the premise of these models the observation of a daysideSO $\mathrm{S}_{2} / \mathrm{SO}$ ratio that is smaller above $86 \mathrm{~km}$ than that inferred for the $74-81 \mathrm{~km}$ region reflects an increase in the photolysis rate of $\mathrm{SO}_{2}$ and a decrease in the rate of oxidation of $\mathrm{SO}$ to $\mathrm{SO}_{2}$ at the higher altitudes.

\subsection{Implied low-latitude dayside vs. terminator behaviors:}


The average of all the $\mathrm{SO}_{2} \mathrm{VMR}$ values inferred from the HST detections obtained on the dayside and in the near-terminator region (i.e. at SZA $<60$ and $\sim 60-75^{\circ}$, respectively) per date of observation is provided in Table IV. On December 28, 2010 the near-terminator $\mathrm{SO}_{2}$ values were $\sim 2 \mathrm{x}$ greater than the average dayside values, replicating the results evident from the comparison of the $\mathrm{SO}_{2}$ column density retrieved at 2-3N latitude at SZAs of $69^{\circ}$ and $40^{\circ}$, respectively. Intriguingly, on January 22, 2011 the average of dayside observations is nearly equivalent to the average of the values obtained at SZAs $\sim 60-75^{\circ}$, in spite of the fact that the $\mathrm{SO}_{2}$ column density retrieved at 27-28S latitude at SZA of $72^{\circ}$ is enhanced by a factor of 1.8 above that observed at SZA=59 . The equivalence of the averaged VMR values emphasizes that the existence and magnitude of the terminator to dayside enhancements may vary strongly with latitude, such that averaging the data at all latitudes washes out enhancements observed in localized regions. Similarly, though there is not strong evidence of an enhancement in the $\mathrm{SO}_{2}$ gas column density on the terminator relative to the dayside detected gas density within a single localized latitude region on January 27, 2011 the average terminator vs. dayside $\mathrm{SO}_{2} \mathrm{VMR}$ results suggests a factor of 2 enhancement in the morning terminator $\mathrm{SO}_{2}$ gas abundance. As discussed above, this behavior most likely reflects the fact that on that date the highest $\mathrm{SO}_{2}$ gas column densities were detected at the highest observed latitudes, and the "high latitude" observations were only made at high SZA. Thus, altogether the three HST observations suggest that if enhancements on Venus' morning terminator occur relative to the dayside $\mathrm{SO}_{2}$ gas abundances, these enhancements are likely to be latitudinally variant, and even may be related to a variance in the overall cloud top $\mathrm{SO}_{2}$ gas abundance.

If the high SZA/"high-latitude” enhancement behavior inferred from the cloud top HST observations continues into the upper regions of the mesosphere, then it would be expected that on some dates the average terminator/near-terminator VMR values at $\mathrm{z} \geq$ $86 \mathrm{~km}$ should be $\sim 2 \mathrm{x}$ greater than the disk-average dayside values inferred from the JCMT dayside observations. For example, on December 28, 2010 the expected magnitude of the average upper mesospheric low-latitude terminator $\mathrm{SO}_{2}$ gas abundances at $\mathrm{z} \geq 86 \mathrm{~km}$ would be $\sim 58 \pm 68 \mathrm{ppb}$; while on (January 28+30, 2011) the expected average upper mesospheric (and dominantly mid-latitude) terminator values would be 
$24 \pm 14 \mathrm{ppb}$. Notably, these hypothetical upper mesospheric terminator values overlap the near-terminator cloud top $\mathrm{SO}_{2} \mathrm{VMR}$ values inferred from the contemporaneously obtained HST observations at the one standard deviation level (Table IV), suggesting that the magnitude of the average $\mathrm{SO}_{2} \mathrm{VMR}$ values at $\mathrm{z} \geq 86 \mathrm{~km}$ may be comparable to the 74-81 km values at all local times. If this is the case, we again emphasize that these similarities do not contradict the basic properties of the 2-layer atmosphere model used to fit the JCMT observations, since the $\mathrm{SO}_{2}$ VMR profile utilized in the fit of the HST data at all local times is assumed to be exponentially decreasing.

Analysis of spatially resolved sub-mm observations of Venus' atmosphere obtained recently with ALMA indicates that an enhancement in the $\mathrm{SO}_{2}$ abundance $\sim 2 \mathrm{x}$ greater than the disk-average values has been observed within the $88-100 \mathrm{~km}$ altitude range at the morning terminator at low-latitudes (Encrenaz et al. 2014). The recent ALMA observations also indicate that the relative balance between the dayside and nearterminator $\mathrm{SO}_{2}$ abundance at low latitudes is temporally varying, to the degree that the actual abundance observed at high and low SZAs can become equivalent or even show an enhancement at low SZA when the overall $\mathrm{SO}_{2}$ gas abundance is low. Thus, the ALMA observations do not contradict the plausibility of our hypothesized low-latitude upper mesosphere dayside vs. terminator gas abundance relationships, while also showing evidence that the observed local time trends may be sensitive to the overall gas abundance in the upper mesosphere, which is undoubtedly dependent on the rate/efficiency of vertical transport (via convection or other mixing processes) of the sulfur-oxide gases from lower altitudes.

New spatially resolved observations of Venus' mesosphere in the 70-100 km region would be the best way to further investigate differences in the average dayside vs. terminator behavior. This would require a combined and coordinated effort of groundbased observing, at multiple wavelengths, so that the full 70-100 km altitude region could be observed without any temporal disparity between observations within any of the observable altitude regimes.

\subsection{Comparison of the implied low-latitude, dayside vs. terminator behaviors to photochemical predictions:}


The Caltech/JPL photochemical model (Allen et al 1981) is used to assess for the first time the expected photochemical steady-state behavior of $\mathrm{SO}_{2}$ in Venus' dayside mesosphere as a function of altitude and solar zenith angle (SZA), in the 58-112 km altitude range, in order to make direct comparison to the dayside vs. terminator $\mathrm{SO}_{2}$ gas abundance trends derived from the December 28, 2010 HST/STIS observations. The 1-d version solves the 1-d continuity equation for all specified species. For these calculations, we assume the same gas-phase constituents and reactions as were utilized in Zhang et al. (2010), but omit the chemical and radiative effects of condensed $\mathrm{H}_{2} \mathrm{SO}_{4}$ and other aerosols; thus, the reactions introduced by Zhang et al. 2010 to induce an $\mathrm{SO}_{2}$ inversion layer in the upper mesosphere are not included in our calculations. The model also does not include the $\mathrm{ClSO}_{2}$ chemistry explored in the Mills et al. (2007) or Mills and Allen (2007) model. The day side temperature, pressure, eddy diffusion, and initial gas number density altitude profiles used in our model are all identical to those utilized in Zhang et al (2010); and, in accordance with the Zhang et al. 2010 model, the mixing ratios for $\mathrm{HCl}$, $\mathrm{CO}, \mathrm{SO}_{2}$, and OCS at $58 \mathrm{~km}$ are specified as $0.4,30,100$, and $0.13 \mathrm{ppm}$, respectively. Unlike Zhang et al. (2010) who diurnally averaged the solar flux at $45^{\circ}$ latitude, our calculations are run to steady-state using the solar flux expected for a specified local time (solar zenith angle) on Venus' equator for December 28, 2010, based on solar flux measurements obtained by SORCE SOLSTICE and SORCE SIM ${ }^{1}$ on 26 December 2010 (Harder et al, 2010; Snow et al, 2005) and assuming a plane parallel atmosphere. This is reasonably accurate for $\mathrm{SZA} \leq 70^{\circ}$, which is also the range of SZA at which the atmosphere was observed by HST at high S/N on December 28, 2010. Production of the gas phase of $\mathrm{H}_{2} \mathrm{SO}_{4}$, which is modeled as occurring via the reaction $\mathrm{SO}_{3}+\mathrm{H}_{2} \mathrm{O} \rightarrow \mathrm{H}_{2} \mathrm{SO}_{4}$ with rate coefficient of $10^{-16} \mathrm{~cm}^{3} \mathrm{~s}^{-1}$, is considered a permanent loss from the simulated system. However, like Zhang et al. (2010) our calculations suggest that the cloud top (74-81 km) $\mathrm{SO}_{2}$ scale height is $\sim 5 \mathrm{~km}$, which is comparable but larger than the $3 \pm 1 \mathrm{~km}$ value derived by $\mathrm{Na}$ et al. (1994) in the analysis of $\mathrm{SO}_{2}$ cloud top (40 mbar, $66 \pm 3 \mathrm{~km}$ ) measurements made in 1988 and 1991.

\footnotetext{
${ }^{1}$ The SORCE SOLSTICE and SORCE SIM data were downloaded from http://lasp.colorado.edu/lisird/ on September 22, 2014. Version 13 SOLSTICE data were used at 115-310 $\mathrm{nm}$ and version 20 SIM data at 310-800 $\mathrm{nm}$
} 
On December 28, 2010, an average $\mathrm{SO}_{2}$ gas number density of $5.3 \times 10^{10} \mathrm{~cm}^{-3}$ was detected at $74-81 \mathrm{~km}$ in Venus' $16-30 \mathrm{~S}$ latitude region at SZAs between $70-90^{\circ}$ by HST/STIS and SOIR. Additionally, on this date, a 2.1 \pm 0.3 terminator enhancement factor is inferred from the $\mathrm{SO}_{2}$ gas column densities detected by HST at 2-3N latitude at SZA $70^{\circ}$ and $40^{\circ}$ on Venus' morning quadrant; and from the average near-terminator (averaging over SZAs $\sim 60-70^{\circ}$ ) vs. average dayside (averaging over SZAs $\sim 25-59^{\circ}$ ) $\mathrm{SO}_{2}$ gas column densities detected by HST (see Table IV). Presuming that this same enhancement factor was included in the gas density detected at $\sim 16 \mathrm{~S}$, SZA $70^{\circ}$, then the anticipated average dayside value (i.e. averaging over SZAs $0-60^{\circ}$ ) value at $16 \mathrm{~S}$ should be $\sim 2.6 \times 10^{10} \mathrm{~cm}^{-3}$.

In Figure 18, we show the results of the SZA-dependent calculations for $45^{\circ}$ and $70^{\circ}$, where the former is representative of the average morning quadrant value and the latter the value anticipated for the near-terminator region. The average $74-81 \mathrm{~km}$ model $\mathrm{SO}_{2}$ number density at SZA $45^{\circ}$ is $1.8 \times 10^{10} \mathrm{~cm}^{-3}$, which is slightly smaller but comparable to the expected dayside value. At SZA $70^{\circ}$, the average model $\mathrm{SO}_{2}$ number density is $5.5 \times 10^{12} \mathrm{~cm}^{-3}$, much larger than the $5.3 \times 10^{10} \mathrm{~cm}^{-3}$ inferred from the HST observations. Thus, the model implies an enhancement in $\mathrm{SO}_{2}$ should be expected at the terminator; although, the calculated degree of enhancement in the 74-81 km range is two orders of magnitude larger than the value inferred from the HST observations.

Figure 18 additionally shows that an enhancement between the dayside and nearterminator equatorial $\mathrm{SO}_{2}$ number density is expected at all altitudes; however, the predicted high SZA near-terminator enhancement is extremely exaggerated in the 70-83 $\mathrm{km}$ altitude region, ranging from a factor of 5 to 600 times greater than the values predicted from simulations run at a SZA of $45^{\circ}$. This behavior highlights that the assumption of photochemical steady state between $\mathrm{SO}$ and $\mathrm{SO}_{2}$ breaks down at high SZA within the lower end of the70-83 km altitude range (see Fig. 19, left panels). Although the primary model losses for $\mathrm{SO}_{2}$ in the mesosphere and lower thermosphere are photodissociation to $\mathrm{SO}$ and oxidation to $\mathrm{SO}_{3}$ [Fig. 19], the former is much faster than the latter. As a result, photo-dissociation of $\mathrm{SO}_{2}$ is the dominant $\mathrm{SO}_{2}$ loss mechanism at altitudes $>73 \pm$ a few $\mathrm{km}$; while at lower altitudes, where the UV photon flux has been diminished, the loss of $\mathrm{SO}_{2}$ via $\mathrm{SO}_{3}$ oxidation is dominant. Thus, in the $70-83 \mathrm{~km}$ altitude 
region it is the transition from a photochemically steady to a non-steady state between SO and $\mathrm{SO}_{2}$ that most critically impacts the predicted $\mathrm{SO}_{2}$ gas density as a function of SZA.

Notably, our calculations assume that nucleation, evaporation, and condensation can largely be ignored. In this case, photochemical equilibrium should hold whenever the photochemical timescale is shorter than the horizontal and vertical transport timescales. Thus, to fully understand the balance of dynamical and chemical processes that impact the $\mathrm{SO}_{2}$ vertical profiles presented in Figure 18, we show in Figure 19 the ratio of the timescales of the key $\mathrm{SO}_{2}$ photochemical loss processes to the timescales of the dynamical transport processes. Although the $\mathrm{SO}_{2}$ photo-dissociation rate is faster than the $\mathrm{SO}_{2}$ oxidation rate, even at those altitudes where photochemical loss of $\mathrm{SO}_{2}$ is dominated by $\mathrm{SO}_{2}$ photo-dissociation, the respective timescales for $\mathrm{SO}$ and $\mathrm{SO}_{3}$ formation must both be considered relative to the transport timescales. Additionally, the chemical cycling of SO is quite complex, below $88 \mathrm{~km}$ at 0 to $70^{\circ} \mathrm{SZA}$, the primary loss for SO is oxidation to $\mathrm{SO}_{2}$, so $\mathrm{SO}_{2}$ that is photo-dissociated to form $\mathrm{SO}$ rapidly recycles back to become $\mathrm{SO}_{2}$. This close linkage is why $\mathrm{SO}+\mathrm{SO}_{2}$ is often referred to as $\mathrm{SO}_{\mathrm{x}}$. Above $88 \mathrm{~km}$ at 0 to $70^{\circ}$ $\mathrm{SZA}, \mathrm{SO}_{\mathrm{x}}$ must to be defined as $\mathrm{S}+\mathrm{SO}+\mathrm{SO}_{2}$ because the primary loss for $\mathrm{SO}$ is photodissociation to $\mathrm{S}$ while oxidation of $\mathrm{SO}$ to $\mathrm{SO}_{2}$ is the second most important loss. This extended definition of $\mathrm{SO}_{\mathrm{x}}$ is appropriate because at and above $64 \mathrm{~km}$ at 0 to $70^{\circ} \mathrm{SZA}, \mathrm{S}$ recycles rapidly back to SO due to the primary loss for $\mathrm{S}$ being oxidation to SO. Additionally, at those altitudes wherein the dominant loss for $\mathrm{SO}_{2}$ is oxidation to $\mathrm{SO}_{3}$, the dominant loss mechanism for $\mathrm{SO}_{3}$ is production of $\mathrm{H}_{2} \mathrm{SO}_{4}$; notably, once the sulfuric acid is formed we assume in our model that it condenses and is lost from the local gasphase chemistry.

Taking all of these things into consideration, comparison of the left and right panels of Figure 19 shows that the specific altitude above which $\mathrm{SO}_{2}$ photo-dissociation to $\mathrm{SO}$ is the dominant $\mathrm{SO}_{2}$ loss mechanism is variable and increases with SZA. This is because optical depth unity is reached at a higher altitude due to the longer path length for solar photons at larger SZA. Our calculations indicate this transition altitude increases from near $70 \mathrm{~km}$ for $45^{\circ} \mathrm{SZA}$ to near $76 \mathrm{~km}$ for $70^{\circ} \mathrm{SZA}$; thus, at $45^{\circ} \mathrm{SZA}$ above $70 \mathrm{~km}$ loss of $\mathrm{SO}_{2}$ via photo-dissociation dominates while below $70 \mathrm{~km}$ loss of $\mathrm{SO}_{2}$ via 
oxidation to $\mathrm{SO}_{3}$ dominates. Similarly, at $70^{\circ} \mathrm{SZA}$ above $76 \mathrm{~km}$ loss of $\mathrm{SO}_{2}$ via photodissociation dominates while below $76 \mathrm{~km}$ loss of $\mathrm{SO}_{2}$ via oxidation to $\mathrm{SO}_{3}$ dominates. Consistent with this, the calculated photochemical loss timescales for $\mathrm{SO}_{2}$ in the lower mesosphere increase from 45 to $70^{\circ} \mathrm{SZA}$. For example, at $45^{\circ} \mathrm{SZA}$ at $74 \mathrm{~km}$ the loss of $\mathrm{SO}_{2}$ is dominated by photo-dissociation to $\mathrm{SO}$, and the calculated $\mathrm{SO}_{2}$ chemical loss timescale is $3.4 \times 10^{3} \mathrm{~s}$; at $70^{\circ} \mathrm{SZA}$ at $74 \mathrm{~km}$, the loss $\mathrm{SO}_{2}$ is now dominated by oxidation to $\mathrm{SO}_{3}$ and the chemical loss time scale increases to $1.4 \times 10^{7} \mathrm{~s}$.

The bottom-right panel of Figure 19 shows that the timescale for $\mathrm{SO}_{2}$ oxidation to $\mathrm{SO}_{3}$ is shorter than the vertical eddy mixing timescale (i.e., ratios are less than 0 in Figure 19) in a narrow range of altitudes between 68 and $74 \mathrm{~km}$ at 0 to $45^{\circ}$ SZA. This is the same region wherein the $\mathrm{SO}_{2}$ density rapidly decreases with altitude (see Figure 18), and is the same altitude region in which sulfuric acid cloud formation is believed to occur. However, the modeled timescale for $\mathrm{SO}_{2}$ oxidation to $\mathrm{SO}_{3}$ is always at least an order of magnitude longer than the timescale for zonal transport.

Notably, the current calculations assume the zonal wind speed is a constant at all altitudes and all SZA and that the vertical eddy mixing profile is the same at all SZA. For completeness we point out that low-latitude zonal wind speeds in the range 70-140 $\mathrm{m} \mathrm{s}^{-1}$ range have been detected between 60-90 km (Piccialli et al 2012, Khatuntsev et al 2013, Kouyama et al 2013); thus, the horizontal transport timescales should vary according to the magnitude of the assumed wind speed. ; Nevertheless, within the range of observed wind speeds the ratio of the photochemical loss timescales to the horizontal timescale is always dominated by the magnitude and sensitivity of the photochemical loss timescale as a function of the SZA; this is the case independent of whether the photochemical loss timescale is defined relative to the $\mathrm{SO}_{2}$ photo-dissociation rate or the $\mathrm{SO}_{3}$ oxidization rate. Thus, within the range of plausible horizontal wind speeds the relative ratio of the $\mathrm{SO}_{2}$ photo-dissociation time scale to the horizontal transport timescale as a function of altitude and SZA follows the exact behavior presented in Figure 19 (top-left panel). Likewise, within the range of plausible horizontal wind speeds, the oxidization timescale is always 1 to a few orders of magnitude longer than the timescale for zonal transport, as is depicted in Figure 19 (top-right panel). 
In summary, Figure 19 shows that the loss of $\mathrm{SO}_{2}$ via $\mathrm{SO}_{3}$ oxidation is always greater than the horizontal transport rates, it is also greater than the vertical transport at all altitudes for $\mathrm{SZA} \geq 50^{\circ}$ and, it is greater than the vertical transport at $\mathrm{z}>72 \mathrm{~km}$ for all $\mathrm{SZA}<50^{\circ}$. Thus, it follows that at those altitudes where the timescale for the loss of $\mathrm{SO}_{2}$ via photo-dissociation is smaller than the horizontal and vertical transport rate, the $\mathrm{SO}_{2}$ photo-dissociation timescale is also smaller than the $\mathrm{SO}_{3}$ oxidation rate. And whenever this is the case, the loss of $\mathrm{SO}_{2}$ via photo-dissociation is the dominant loss mechanism.

Therefore, we continue to emphasize that the interpretation of the results presented in Figure 18 at altitudes $\geq 73 \pm$ a few $\mathrm{km}$ are most directly dependent on the relative timescales for photo-dissociation of $\mathrm{SO}_{2}$ and transport at $\mathrm{SZAs}$ ranging from 0 to $70^{\circ}$, and the resulting transition of the $\mathrm{SO}_{\mathrm{x}}$ species from a steady to a non-steady state. The $\mathrm{SO}_{2}$ photo-dissociation timescale at $78-100 \mathrm{~km}$ is in the range of $2.2-7.7 \times 10^{3} \mathrm{~s}$ for SZAs in the range of $45-70^{\circ}$; the vertical eddy diffusion transport timescale is $1.5-10 \times 10^{5}$ $\mathrm{s}$; and the zonal transport timescale remains $\sim 1 \times 10^{4} \mathrm{~s}$. Thus, above $78 \mathrm{~km}$ altitude, the $\mathrm{SO}_{2}$ photo-dissociation timescale is shorter than both the vertical eddy mixing and zonal transport timescales at all $\mathrm{SZA}$, so $\mathrm{SO}_{2}$ and $\mathrm{SO}$ should be in photochemical equilibrium with each other. Below $68 \mathrm{~km}$ altitude, the relative balance between the photochemical and transport timescales at all SZAs is reversed thus, $\mathrm{SO}_{2}$ and $\mathrm{SO}$ should not be in photochemical equilibrium with each other. At the intervening altitudes, $68-78 \mathrm{~km}, \mathrm{SO}_{2}$ and SO are expected to be in photochemical equilibrium with each other at smaller SZA but not at larger SZA. Most of the transition occurs between 45 and $70^{\circ}$ SZA. In particular, at $45^{\circ} \mathrm{SZA}$ the entire $74-81 \mathrm{~km}$ region observed by HST is expected to have $\mathrm{SO}_{2}$ and $\mathrm{SO}$ in photochemical equilibrium with each other, while at $70^{\circ} \mathrm{SZA}$ only the upper part of this region, which has smaller $\mathrm{SO}_{2}$ abundances, is expected to have $\mathrm{SO}_{2}$ and SO in photochemical equilibrium with each other. Consequently, the enhancement observed by HST in the 74-81 km region at $70^{\circ}$ SZA should be, and is, smaller than that predicted by our steady-state calculations.

On the other hand, at 88-100 km, the predicted terminator enhancement factor is $2.7 \pm 0.2$, which is comparable to the factor of 2 enhancement in the near-terminator upper mesospheric /lower thermospheric $\mathrm{SO}_{2}$ gas mixing ratio that was inferred for 88-100 km 
altitude region from spatially resolved ALMA observations obtained in 2012 subsequent to the December 28, 2010 HST and SOIR observations (Encrenaz et al. 2014). As indicated above, at these altitudes $\mathrm{SO}_{2}$ and $\mathrm{SO}$ should be in photochemical equilibrium with each other. Thus, the new exploration of the photochemical schemes relative to a specified local time emphasizes that $\mathrm{SO}_{2}$ and $\mathrm{SO}$ are not likely to be in photochemical steady state with each other at SZA $>\sim 60^{\circ}$ in the lower mesosphere (below $\sim 75 \pm 2 \mathrm{~km}$ ), but that in the upper mesosphere (above $78 \mathrm{~km}$ ) $\mathrm{SO}_{2}$ and $\mathrm{SO}$ should be in photochemical steady state with each other even at high SZAs. These calculations are the first to define the conditions where photochemical steady state for $\mathrm{SO}_{2}$ and $\mathrm{SO}$ can be assumed in Venus' mesosphere.

The steady-state vertical $\mathrm{SO}_{2}$ mixing ratio profiles presented in Figure 18 indicate that $\mathrm{SO}_{2}$ is transported upward from the 58-km lower boundary of the model until photodissociation induces a rapid loss with increasing altitude. Additionally, the current calculations highlight that the $\mathrm{SO}_{2}$ concentration (and mixing ratio) increase with SZA. The inter-dependency of these properties on the SZA is logical but has not and could not be uniquely inferred from previous simulations that have not considered the variation of the solar flux with SZA. Thus, the work presented here represents the first steps in developing a photochemical model that accurately predicts the diurnal variation of Venus' mesospheric gas chemistry, which is inherently a function of the diurnal variation of the photon flux as well as other factors, such as zonal transport. Future modeling work will consider the combined effects of vertical transport, zonal transport, and the diurnallyvarying photon flux to examine the diurnal behavior of $\mathrm{SO}_{2}$ and $\mathrm{SO}$ in a more realistic manner, rather than assuming photochemical steady state at all altitudes.

\section{Summary and Future Projections}

The HST data provide the first direct and simultaneous measure of Venus' mesospheric cloud top ( $\mathrm{z} \sim 75 \pm 2 \mathrm{~km}$ ) $\mathrm{SO}$ and $\mathrm{SO}_{2}$ gas column density variability as a function of latitude and time of day. Analysis of the HST observations confirms the longstanding trend that the $\mathrm{SO}_{2}$ gas column density at the cloud tops is strongly latitudinally dependent (c.f. Na et al. 1994, Encrenaz et al. 2012, Marcq et al. 2013), and for the first time definitively shows that the cloud top SO gas column density distribution is directly 
correlated with the cloud top $\mathrm{SO}_{2}$ gas density-rather than anti-correlated as would be expected if the $\mathrm{SO}_{\mathrm{x}}$ balance was purely photochemically driven and the $\mathrm{SO}_{2}$ and $\mathrm{SO}$ were the primary/solitary sources for the sulfur reservoir at these altitudes. The $\mathrm{SO}_{2}$ and $\mathrm{SO}$ VMR values recently retrieved for the altitude range $\geq 86 \mathrm{~km}$ from sub-mm observations also show that an additional sulfur reservoir should be present in the upper mesosphere (Sandor et al., 2010). Thus, the HST results extend the altitude range through which it should not be assumed that the $\mathrm{SO}_{2}$ and $\mathrm{SO}$ gases are the solitary sources for Venus' atmospheric sulfur reservoir.

Our analysis (like that of the nadir VEx/SPICAV-UV observations) further indicates that the direction of relative increase in the $\mathrm{SO}_{2}$ and $\mathrm{SO}$ gas densities with latitude is variant. On two of the three days the $\mathrm{SO}_{2}$ and $\mathrm{SO}$ gas column density is observed to peak at the equator, however, on one of the three days the gas densities are observed to increase with increasing latitude. For the former two dates, a factor of $2.1 \pm 0.3$ enhancement in the $\mathrm{SO}_{2}$ gas density near the terminator (i.e., at $\mathrm{SZA} \sim 70^{\circ}$ ) is observed relative to the gas density detected at equivalent latitude but smaller SZA. However, no evidence of any enhancement at high SZA independent of the increase in gas density with increasing latitude is evident for the third date at which time the reversed latitudinal gradient was also observed.

Independent of the observed reversal in the latitudinal variation gradient, both the equatorial $\mathrm{SO}_{2}$ and $\mathrm{SO}$ gas column densities observed by HST between late December 2010 and February 2011 became progressively lower on the dayside (i.e. at SZA $<60^{\circ}$ ) at low latitudes. In particular, the $\mathrm{SO}_{2}$ gas density inferred from the January 27, 2011 HST observation at $\pm 15^{\circ}$ latitude was a factor of $\sim 2$ 2-3 and $\sim 20$ lower that that inferred from the January 22, 2011 and December 28, 2010 observations, respectively. The $0.245 \pm 0.004 \mu \mathrm{m}$ cloud top brightness at those latitudes was also observed to decline coincidently with the gas densities; and comparison of the VMC observations taken contemporaneously with the January 2011 observations on the southern hemisphere (from $\sim 7 \mathrm{~S}$ to $90 \mathrm{~S}$ ) indicates that the $0.36 \mu \mathrm{m}$ cloud top brightness was darker on January 27 northward of 35S than that observed on January 22, 2011, and that the latitudinal gradient of the cloud top brightness also changed slope northward of $\sim 20 \mathrm{~S}$ on that date. 
Though the HST observing sample is small, the observed $\mathrm{SO}_{2}$ variation behavior replicates the dayside behavior inferred from SPICAV-UV nadir observations taken throughout the lifetime of the VEx mission. In particular, both HST and SPICAV-nadir observed a general decline in the low-latitude dayside $\mathrm{SO}_{2}$ gas density retrievals and $\sim$ $0.24 \mu \mathrm{m}$ cloud top brightness between late December 2010 and April 2011. The $\sim 0.24 \mu \mathrm{m}$ darkening observed by HST and SPICAV suggests that the sub-micron particle density decreased (either due to a lower influx or due to the coalescence of the particles into larger particles) over the observation time period. Additionally, the average dayside $\mathrm{SO}_{2}$ VMR value inferred from the HST observations in the time period between December 2010 and February 2011 is in the range of $42 \pm 36$ ppb, which is identical to the average dayside VMR value derived from the SPICAV-UV data for the entirety of 2011, and 10x lower than the average value observed in 2007 (see Fig. 2). And lastly, the long-term average behavior recorded in the 2006-2013 SPICAV-UV nadir observations indicates a decrease in the $\mathrm{SO}_{2}$ gas column density with increasing latitude is the "normal state" of the latitudinal variation, but the reversed $\mathrm{SO}_{2}$ latitude gradient trend is evident when the lowest equatorial $\mathrm{SO}_{2}$ gas column density is detected (Marcq et al. 2013). This same trend is replicated by the HST observations.

Comparison of the $\mathrm{SO}_{2}$ VMR values derived from HST observations and SOIR observations obtained contemporaneously on December 28, 2010 near/at the terminator at $\sim 20 / 31 \mathrm{~S}$ indicates that both observations were sensitive to the $\mathrm{SO}_{2}$ gas density present between 74-81 km, that the retrieved $\mathrm{SO}_{2}$ VMRs were $\sim 250-290 \mathrm{ppb}$ (Mahieux et al. 2014), and that these relatively high mixing ratios were obtained because the $\mathrm{CO}_{2}$ densities inferred from the 2010 SOIR observations were $~ 1.4 \mathrm{x}$ smaller than those in the VIRA catalog. The $\mathrm{SO}_{2}$ VMR morning terminator values inferred from the HST and SOIR observations obtained on 12/28/2010 correspond to the highest values observed by SOIR in the 2006-2013 time period at latitudes $\leq 40^{\circ}$ from the equator. Conversely, the 6-20 ppb (assuming $\mathrm{CO}_{2}$ profile provided by VIRA) near-terminator $\mathrm{SO}_{2}$ VMRs inferred from the two January 2011 observations intersect the lowest range of the low-latitude 75$78 \mathrm{~km}$ terminator $\mathrm{SO}_{2} \mathrm{VMR}$ values inferred from the SOIR observations over the lifetime of the VEx mission. Intriguingly, there was limited difference in the near-terminator $\mathrm{SO}_{2}$ VMR values inferred from the two January 2011 observations. The sensitivity of the low- 
latitude $\mathrm{HST} \mathrm{SO}_{2}$ gas density detections to the $75-78 \mathrm{~km}$ altitude range reflects the relationship of the $\mathrm{SO}_{2}$ vertical profile to the aerosol profile of the large ( $1 \mathrm{um}$ ) particles. Although the exact mechanism responsible for the observed variability is unconstrained, both the $\mathrm{SO}_{2}$ and aerosol profiles are strongly linked to vertical mixing in the atmosphere (c.f. Esposito and Travis, 1982); thus, the near-terminator $\mathrm{SO}_{2}$ abundance retrievals seem to suggest that vertical mixing was stronger in late 2010 than in late 2011. In reality, drivers for a change in the upward vertical transport may well be dynamically or chemically driven. For example, a change in the vertical temperature profile could lead to a change in the vertical eddy diffusion rates; or alternatively, the observed variability may indicate that the $\mathrm{H}_{2} \mathrm{SO}_{4}$ production/loss rate was perturbed.

The HST observed decline in the equatorial $\mathrm{SO}_{2}$ gas abundance, followed by a reversal in the latitudinal variation of the dayside $\mathrm{SO}_{2}$ gas distribution within Venus' 5 day rotation cycle, and the corresponding stability of the low-latitude $\mathrm{SO}_{2}$ gas density at high solar zenith angle on the same time scale matches the behaviors predicted by Marcq et al. (2013) for a mesospheric $\mathrm{SO}_{2}$ gas distribution that is maintained by Hadley-cell like gas circulation in balance with photochemical processing, where the ascending node of the Hadley-cell is initiated by vertical mixing at the equator. Similarly, if we presume that the cloud top brightness is directly correlated with the $\mathrm{SO}_{2}$ abundance, then the observed variance of the cloud brightness between 5-20 S latitude, and invariance in the cloud brightness poleward of 35S latitude is also in agreement with the Marcq et al. model. These agreements suggest that the reversal in the dayside low-latitude $\mathrm{SO}_{2}$ latitudinal gradient evident between the two January 2011 observations may have been driven by a decrease in the upward vertical transport (perhaps due to a change in the vertical extent of the convective cells that define the Hadley circulation or due to a change in the eddy diffusion (see Marcq et al. 2013, Krasnopolsky et al. 2012)) at the equator on January 22, 2011, and that the rate of upward vertical transport operating in the atmosphere between January 22 and January 27, 2011 was insufficient to maintain the typical $\mathrm{SO}_{2}$ latitudinal gradient inferred from the SPICAV observations - this in turn implies that the vertical transport rate operating during the January 2011 HST observations was significantly less than the average rate. Thus, both the dayside and near-terminator January 2011 retrievals suggest that vertical transport in late January 2011 was significantly less than the highest 
or even the average rates operating during the lifetime of the VEx mission. At the same time, the progressive decline in the $0.24 \mu \mathrm{m}$ brightness of the cloud tops inferred from the HST and SPICAV-nadir observations implies that the density of the sub-micron (UV bright) haze decreased within the time period of the two datasets. Together, these two results imply a correlation between vertical transport rate and Venus' mesospheric submicron haze density.

The combination of the 2006-2013 VEx/SPICAV-UV observations with previous measurements of the cloud top $\mathrm{SO}_{2}$ mixing ratios indicates that Venus' dayside $\mathrm{SO}_{2}$ gas abundance tends to vary cyclically, declining over multiple years before increasing again (Fig. 2). Therefore, continued monitoring of Venus' cloud top $\mathrm{SO}_{2}$ gas abundance over multiple years is needed to keep an accurate record of the cyclical pattern in the average dayside $\mathrm{SO}_{2}$ gas density present at low-latitudes above Venus' cloud tops. In fact, both the SPICAV and HST observations indicate that the low-latitude dayside $\mathrm{SO}_{2}$ cloud top gas density late in the Venus Express mission was on average $<5 \mu \mathrm{m}$-atm (e.g. the average dayside value derived from the 2011 HST data taken directly at the equator is $0.9 \mu \mathrm{m}$-atm, see Table IV, Fig. 12); additionally, the long-term SPICAV observations indicate that the cloud-top $\mathrm{SO}_{2}$ abundance decreases by only $30 \%$ within of year of a detected $\mathrm{SO}_{2}$ VMR spike (Fig. 2). Thus, continued observation of Venus' cloud-top $\mathrm{SO}_{2}$ gas density beginning within a year of the end of the Venus Express mission is especially critical for accurately identifying when/if a new spike in the $\mathrm{SO}_{2}$ cloud top behavior density occurs. The results presented here indicate that HST observations can be used to infer details about the physical processes that drive the short term variability in Venus' mesospheric $\mathrm{SO}_{2}$ gas density, and may be useful for documenting the average lowlatitude dayside $\mathrm{SO}_{2}$ and $\mathrm{SO}$ gas densities within a single year (Fig. 2). And, as is true for the VEx imaging observations, the detailed spectral measurements provided by HST would be an added benefit to any pictorial imaging of the cloud top UV contrast achievable from any future mission to Venus including the Akatsuki mission, which will re-attempt insertion into Venus orbit in December 2015, but has no spectroscopic instruments included in its payload. In fact, since the end of VEx mission in late 2014, HST provides the only means to obtain spectroscopic observations of Venus' cloud tops at UV wavelengths on an on-going basis. Likewise, since the loss of the VEx mission, 
ground/Earth based observations are the only platform through which spectroscopic observation of Venus in the UV-visible-IR range may be obtained in the foreseeable future.

7.1 Advances in Photochemical Modeling: The coordinated observing effort presented here provides measurements that are useful for developing and assessing photochemical modeling schemes that explore the expected average dayside and nearterminator vertical profile behavior within Venus’ mesosphere, and distinguish the key physics supporting the observed behaviors. In particular, the available contemporaneous observations using HST and JCMT have provide dayside average vertical profiles spanning most of Venus' upper mesosphere, while the combined HST and SOIR observations provide insights on the terminator/near-terminator behavior in the $74-81 \mathrm{~km}$ range, which corresponds to the first few kilometers of Venus' upper mesosphere. Thus, the $\mathrm{SO}_{2} / \mathrm{SO}$ ratio is defined over two specific altitude ranges wherein the response to variations in Venus' photochemical timescale with SZA are distinctive; as a result, the altitude regions where the relative import of photo-dissociation and oxidation are distinctly different can be fully defined and characterized. For example, comparison of the contemporaneously obtained average dayside detections with standard photochemical models for the partitioning of $\mathrm{SO}_{2}$ and SO (Sandor et al 2010, Zhang et al 2012, Belyaev et al 2012), suggests that the smaller dayside $\mathrm{SO}_{2} / \mathrm{SO}$ ratio observed in the upper mesosphere at $\mathrm{z} \geq 86 \mathrm{~km}$ reflects an increase in the photolysis rate of $\mathrm{SO}_{2}$ and a decrease in the rate of oxidation of $\mathrm{SO}$ to $\mathrm{SO}_{2}$ at these higher altitudes, independent of the source of the upper mesospheric sulfur oxides.

Additionally, comparison of the expected steady state photochemical behavior at $\mathrm{SZA}=45^{\circ}$ (representative of the average dayside on the morning quadrant) vs. $\mathrm{SZA}=70^{\circ}$, (representative of the near-terminator) indicates that the morning terminator should be enhanced relative to the dayside, and that this behavior is evident both in the $74-81 \mathrm{~km}$ region, and at higher altitudes. The new exploration of the photochemical behaviors relative to the solar flux available at a specified local time emphasizes that photochemical equilibrium between $\mathrm{SO}_{2}$ and $\mathrm{SO}$ is not likely above $\mathrm{SZA} \sim 60^{\circ}$ in the lower mesosphere; that above $78 \mathrm{~km}$ the $\mathrm{SO}_{2}$ gas abundance is basically steady; and that the near-terminator $\left(\mathrm{SZA} \sim 70^{\circ}\right) \mathrm{SO}_{2}$ abundance is predicted to be enhanced by a factor of $\sim 2.7 \pm 0.2$ at $\mathrm{z} \geq 88$ 
$\mathrm{km}$ over the abundance levels expected at smaller SZAs, which is comparable to the factor of 2 enhancement inferred from spatially resolved sub-mm ALMA observations obtained at those altitudes subsequent to the presented coordinated observing effort (Encrenaz et al. 2014).

The contemporaneous HST UV-nadir and SOIR near-IR limb terminator observations may also provide an unique opportunity to investigate the photochemistry of $\mathrm{SO}_{2}$ across the terminator. I.e., in general, high SZA 200-260 nm HST observations constrain the near-terminator, sunlit chemistry at $\sim 73 \pm 5 \mathrm{~km}$; and, the analysis of the current data set indicates strong similarities between SZAs of $70-90^{\circ}$. Photochemical models that attempt to combine the HST data with SOIR's 70-85 $\mathrm{km} \mathrm{SO}_{2}$ vertical profiles, which typically integrate over roughly 30 minutes of local time on each side of the terminator, may provide new insights into Venus' cloud-top cross-terminator behavior. Because simultaneous limb and nadir soundings are required to gather this type of information, now that the Venus Express mission has ended, unless a limb-sounding instrument becomes available in the near future the existing contemporaneous HST and SOIR dataset may provide the only opportunity for a cross-terminator analysis of $\mathrm{SO}_{2}$ in the 70-80 km altitude region uniquely sampled by the UV observations, since HST's remaining operational lifetime is limited.

The current coordinated observing effort provides the first availability of $\mathrm{SO}_{\mathrm{x}}$ gas distributions with high latitudinal and local time resolution within specific altitude regimes. While the opportunity to simulate this type of data represents a step forward in photochemical modeling, the availability of spatially-resolved contemporaneously obtained data providing vertical profiles spanning most of the mesosphere would be quite useful for developing and assessing photochemical modeling schemes that can consider more specifically the impact of the available photon flux on the diurnal gas chemistry behavior, and secondary reactions such as the condensation and evaporation of $\mathrm{H}_{2} \mathrm{SO}_{4}$. In fact, 3-D maps of the $\mathrm{SO}$ and $\mathrm{SO}_{2}$ behavior, extending from $60-100 \mathrm{~km}$ on a daily basis within Venus' 4-5 day rotation period would provide an unprecedented window into the balance among the photolytic, kinetic and dynamic drivers that control sulfur oxide chemistry in Venus' mesosphere on the dayside and near the dawn and dusk terminator regions. Such observations can be obtained, but would require a coordinated effort 
between instruments that can obtain spatial resolution horizontally across the disk with adequate altitude resolution. E.g., a coordinated effort to obtain HST spectral and imaging observations that could be used to map the $\mathrm{SO}$ and $\mathrm{SO}_{2}$ gas density behavior as a function of latitude, and time of day in the 70-80 km region, in conjunction with mid-IR TEXES observations and sub-mm ALMA observations that sense the 60-70 km and 88$100 \mathrm{~km}$ altitude regions, respectively, would provide the necessary altitude coverage at high spatial resolution from which the key processes regulating Venus' mesospheric sulfur-oxide chemistry can be discerned on the dayside and near the dawn and dusk terminator regions. Additionally, because of the sensitivity of the sub-mm and IR observations to the nightside behavior, once the $60-100 \mathrm{~km}$ dawn to dusk dayside behavior is well understood, predictions regarding the 60-100 km nightside behavior can also be developed.

7.2 Cloud modeling efforts: The aerosol distribution utilized in our model directly impacts our inference of the altitude of the detected $\mathrm{SO}_{2}$ gas column density, which in turn impacts our interpretation of the relative significance of the roles of $\mathrm{SO}_{2}$ photolysis, oxidation and recombination as a function of altitude as well as our understanding of the abundance of $\mathrm{SO}_{2}$ available to contribute to the formation/loss of the sulfur bearing aerosol species within Venus' atmosphere. Analysis of SPICAV-IR occultation observations (Wilquet et al. 2009, Wilquet et al. 2012) indicates that the upper boundary of the mode $2(\mathrm{r}=1.01 \mu \mathrm{m})$ particles may be higher than the typical low-latitude cloud height inferred from nadir $\mathrm{CO}_{2}$ absorption observations obtained at $1.6 \mu \mathrm{m}$ or from 0.36 $\mu \mathrm{m}$ cloud top brightness imaging (Ignatiev et al. 2009, Titov et al. 2012). This implies that the Mie scattering extinction at altitudes greater than the $\mathrm{CO}_{2}$ optical depth unity altitude may be more significant than previously assumed, and that the total aerosol optical depth unity altitude is higher than that inferred solely from the $\mathrm{CO}_{2}$ absorption behavior.

Currently, in our simple model the density of the mode 1 particles is assumed to be constant below the $\mathrm{CO}_{2}$ optical depth unity altitude, and because this altitude is presumed to be the altitude of the upper cloud boundary, the mode 2 particles are assumed to only be present below $\mathrm{CO}_{2}$ optical depth unity altitude. Consequently, in the model, the magnitude of the Mie scattering extinction does not change as rapidly with 
altitude as the $\mathrm{CO}_{2}$ Rayleigh scattering, and is dependent only on the mode 1 particles above the UCB. We believe that modifying the current cloud aerosol distribution in the model to allow for a more realistic distribution of the mode 1 and mode 2 particles, including utilizing separate definitions for the mode 1 and mode 2 aerosol scale heights will lead to an improved understanding of the relationship between the aerosol scattering signatures and the gas absorption signatures. However, any changes to the cloud model must be based on improved understanding of the cloud properties derived from the Venus Express mission. Development of a new cloud model based on the Venus Express mission is on-going, and is being completed in conjunction with the SPICAV-IR and VMC teams.

\section{Acknowledgements:}

The authors are grateful to Marty Snow of the Laboratory for Atmospheric and Space Physics at University of Colorado, Boulder for providing SOLSTICE data at high spectral $(0.33 \AA$ ) $)$ sampling. The authors are grateful to Brad Sandor for coordinating and acquiring the JCMT data, and hours of useful conversation and discourse. Investigator Sandor was supported by the U.S. National Science Foundation under Grant no. AST1312985, and by NASA under Grant nos. NNX10AB33G, NNX12AI32G, and NNX14AK05G toward completion of this research.

The authors also acknowledge the James Clerk Maxwell Telescope which has historically been operated by the Joint Astronomy Centre on behalf of the Science and Technology Facilities Council of the United Kingdom, the National Research Council of Canada and the Netherlands Organization for Scientific Research.

The authors also thank the International Space Science Institute (ISSI) for their fruitful support of the documentation of these observations. Most of the authors of this manuscript were members of the ISSI International Team "Sulfur Dioxide variability in the Venus atmosphere" who met during the 2013-2015 in the facilities of ISSI in Bern, Switzerland.

Finally the authors are most grateful to Adriana Ocampo, NASA Headquarters, John Grunsfield, NASA Headquarters, Alan Stern, SwRI, Claus Leither, Space Telescope Science Institute, Hvakam Svedhem, Venus Express Project Scientist, and the VEx Science Working Team, for their support in the acquisition and analysis of the HST observations obtained through NASA/HST program 12433. This research was funded through the NASA Early Careers Program, NASA Grant NNX11AN81G; the NASA Planetary Atmospheres Program, Grant NNX12AG55G and through a grant from Space Science Telescope Institute, which is operated by the Association of Universities for Research in Astronomy, Inc., under NAS5-26555.

\section{References}


Allen, M, Y.L. Young and J. W. Waters, 1981. Vertical transport and photochemistry in the terrestrial mesosphere and lower thermosphere. J Geophys Res 86 p. 3617-3627.

Belyaev, D., Montmessin, F., Bertaux J-L., Mahieux A., Fedorova A., Korablev O. I., Marcq E., Yung, Y. L., Zhang X.,2012. Vertical profiling of $\mathrm{SO}_{2}$ and SO above Venus’ clouds by SPICAV/SOIR solar occultations. Icarus, 217, p. 740-751.

Blackie, D., Blackwell-Whitehead, R., Stark, G., Pickering, J.C., Smith, P.L., Rufus, J., Thorne, A.P., 2011. Correction to "High-resolution photoabsorption crosssection measurements of $\mathrm{SO}_{2}$ at $198 \mathrm{~K}$ from 213 to $325 \mathrm{~nm}$ '. J. Geophys. Res. 116, E12099, http://dx.doi.org/10.1029/2011JE003977.

Clancy, R.T., Sandor, B.J., Moriarty-Schieven, G.H., 2003. Observational definition of the Venus mesopause: Vertical structure, diurnal variation, and temporal instability. Icarus 161, p. 1-16.

Clancy, R.T., Sandor, B.J., Moriarty-Schieven, G.H., 2008. Venus upper atmospheric $\mathrm{CO}$, temperature, and winds across the afternoon/evening terminator from June 2007 JCMT sub-millimeter line observations. Planet. Space Sci. 56, p. 1344-1354.

Clancy R.T., Sandor, B.J., Moriarty-Schieven, G.H., 2012. Thermal structure and CO distribution for the Venus mesosphere/lower thermosphere: 2001-2009 inferior conjunction sub-millimeter CO absorption line observations, Icarus, 217, pp. 779-793.

Egorova, T., Rozanov, E., Hochedez, J.-F. , and W. Schmutz, 2008. Reconstruction of the solar spectral UV irradiance for nowcasting of the middle atmosphere state on the basis of LYRA measurements, Atmos. Chem. Phys., 8, p. 29652973

Encrenaz, T., T. K. Greathouse, H. Roe, M. Richter, J. Lacy, B. Bézard, T. Fouchet, and T. Widemann, 2012. HDO and $\mathrm{SO}_{2}$ thermal mapping on Venus: Evidence for strong $\mathrm{SO}_{2}$ variability. Astronomy \& Astrophysics, 543, p.1-7 . http://dx.doi.org/ 10.1051/004-6361/201219419

Encrenaz, T., R. Moreno, A. Moullet, E. Lellouch, T. Fouchet, 2014. Submillimeter mapping of mesospheric minor species on Venus with ALMA, Planetary and Space Science., Submitted

Esposito, L.W., 1980. Ultraviolet contrasts and the absorbers near the Venus cloud tops. J. Geophys. Res. 85, pp. 8151-8157. 
Esposito, L.W. and L. D. Travis, 1982. Polarization studies of the Venus UV constrasts: Cloud height and haze variability. Icarus, 51, pp. 374-390, doi:10.1016/001910355(82)90090-2.

Esposito, L.W, Knollengerg, R. G., Marov, M.,Y., Toon, O. B., Turco, R., P.,1983. "The Clouds and Hazes on Venus”, in Venus, Eds., Hansen, Colin, Donahue, Moroz, University of Arizona press, pp. 484-564.

Esposito, L.W., Copley, M., Eckert, R., Gates, L., Stewart, A.I.F., Worden, H.1988. Sulfur Dioxide at the Venus Cloud Tops, 1978-1986. J. Geophys. Res., 93(D5): p. $5267-5276$.

Harder, JW, Thuillier, G., Richard, E. C. , Brown, S. W. , Lykke, K. R., Snow, M., McClintock, W. E., Fontenla, J. M., Woods, T. N., Pilewskie, P., 2010. The SORCE SIM solar spectrum: Comparison with recent observations. Sol Phys 263, p.3-24.

Ignatiev, N.I., Titov, D.V., Piccioni, G., Drossart, P., Markiewicz, W.J., Cottini, V., et al., 2009. Altimetry of the Venus cloud tops from the Venus Express observations. J. Geophys. Res. 114, p. 405-414.

Johnson, N. M. and B. Fegley, 2002. Experimental Studies of AtmosphereSurface Interactions On Venus. Adv. Space Res. 29, pp. 233-241.

Khatuntsev Khatuntsev, I.V.,. Patsaeva , M.V., Titov, D.V., Ignatiev, N.I., Turin, A.V., Limaye, S.S., Markiewicz, W.J., Almeida, M.,. Roatsch, Th., Moissl R, 2013. Cloud level winds from the Venus Express Monitoring Camera imaging. Icarus 226, p. 140-158

Kouyama T.,Imamura, T.,,Nakamura, M., , Satoh, T.,and Futaana, Y., 2013. Long-term variation in the cloud-tracked zonal velocities at the cloud top of Venus deduced from Venus Express VMC images J. Geophys. Res. 118, p. 37-46, http://dx.doi.org/10.1029/2011JE004013

Krasnopolsky, V.A. and Pollack 1994, $\mathrm{H}_{2} \mathrm{O}-\mathrm{H}_{2} \mathrm{SO}_{4}$ system in Venus' clouds and OCS, $\mathrm{CO}$ and $\mathrm{H}_{2} \mathrm{SO}_{4}$ profiles in Venus' troposophere. Icarus, 109, p.58-78.

Krasnopolsky, V.A., 2006. Chemical composition of Venus atmosphere and clouds: Some unsolved problems. Planet. Space Sci., 54, p. 1352-1359.

Krasnopolsky, V.A., 2012. A photochemical Model for the Venus Atmosphere at 47-112 km. Icarus, 218: p. 230-246. 
Mahieux, A., Vandaele, A.C., Drummond, R., Robert, S., Wilquet, V., Fedorova, A., Bertaux, J.L., 2010. Densities and temperatures in the Venus mesosphere and lower thermosphere retrieved from SOIR onboard Venus Express: Retrieval technique. $J$. Geophys. Res. 115, 10.1029/2010JE003589.

Mahieux, A., Vandaele, A.C., Bougher, S.W., Drummond, R., Robert, S., Chamberlain, S., Wilquet, V., Piccialli, A., Montmessin, F., Tellmann, S., Patzold, M., Hausler, B., Bertaux, J.L., 2014a. Update of the Venus density and temperature profiles at high altitude measured by SOIR on board Venus Express. Planet. Space Sci. (submitted)

Mahieux, A., Vandaele, A.C., Robert, S., Wilquet, V., Drummond, R., Belyaev, D., Bertaux, J.L., 2014b. Venus mesospheric sulfur dioxide measurement retrieved from SOIR on board Venus Express. Planet. Space Sci. (submitted)

Marcq, E., Belyaev, B., Montemssin, F., Fedoroav, A., Bertuax, J-L., Vandaele, A.C. and, E. Neefs, 2011. An investigation of the $\mathrm{SO}_{2}$ content of the venusian mesosphere using SPICAV-UV in nadir mode. Icarus, 211: p. 58-69.

Marcq, E., Bertaux, J-L., Montmessin, F. and D. Belyaev., 2013. Variations of sulphur dioxide at the cloud top of Venus's dynamic atmosphere. Nature Geoscience, 6: p. 25-28.

McClintock, W. E., Rottman, G. J., and T. N. Woods, 2005. Solar-Stellar Irradiance Comparison Experiment II (SOLSTICE II): Instrument concept and design, Sol. Phys., 230, p. 225-258, http://dx.doi.org/10.1007/s11207-005-7432-x.

Mills, FP, 1998. I. Observations and photochemical modeling of the Venus middle atmosphere, II. Thermal infrared spectroscopy of Europa and Callisto, PhD dissertation, California Institute of Technology, Pasadena, CA, 366 pp.

Mills, F.P., LW Esposito, and YL Yung, 2007. Atmospheric composition, chemistry, and clouds, in Exploring Venus as a Terrestrial Planet, eds. LW Esposito, ER Stofan, and TE Cravens, 73-100, American Geophysical Union, Washington, DC.

Mills, FP, and M Allen, 2007. A review of selected issues concerning the chemistry in Venus' middle atmosphere, Planetary and Space Science, 55 p. 1729-1740

Molaverdikhani, K. ,McGouldrick, K., Esposito. L. W., 2012. The abundance and vertical distribution of the unknown ultraviolet absorber in the venusian atmosphere from analysis of Venus Monitoring Camera images, Icarus 217 p 648-660.

Na, C.Y., Esposito, L.W., McClintock, W.E., Barth, C.A., 1994. Sulfur dioxide in the atmosphere of Venus. Icarus 112 p. 389-395. 
Nishitani, E., K. Fukuda, and I. Tanaka, 1985. "Photoionization Study of the Sulfur Monoxide Radical Produced by Pyrolysis of Ethylene Sulfoxide," Bulletin of the Chemical Society of Japan 58, 3475-3479.

Petrass, J, 2013. Modelling of sulfur oxide and cloud chemistry in Venus' mesophere, Honours thesis, Research School of Physics and Engineering, Australian National University, Canberra, ACT, Australia, 76 pp.

Phillips, L., 1981. Absolute absorption cross sections for SO between 190 and 235 nm. J. Phys. Chem. 85, 3994-4000.

Piccialli , A., Tellmann, S, Titov, D.V, Limaye, S.S., Khatuntsev , I.V., Pätzold, M.. Häusler, B., 2012. Dynamical properties of the Venus mesosphere from the radiooccultation experiment VeRa onboard Venus ExpressIcarus 217, p. 669-681.

Rossow, W. B., A. D. Del Genio, S. S. Limaye, L. D. Travis, and P. H. Stone, 1980. Cloud morphology and motions from Pioneer Venus images. J. Geophys. Res., 85, pp. 8107-8128, doi: 10.1029/JA085iA13p08107.

Rufus, J., Stark, G., Smith, P.L., Pickering, J.C., Thorne, A.P., 2003. Highresolution photoabsorption cross section measurements of SO2, 2: 220 to $325 \mathrm{~nm}$ at 295 K. J. Geophys. Res. 108 (E2), 5-1. http://dx.doi.org/10.1029/2002JE001931 (CiteID 5011).

Rufus, J. , Stark, G., Thorne, A. P., Pickering, J. C., Blackwell-Whitehead' R. J., Blackie, D. and P. L. Smith,, 2009. High-resolution photoabsorption cross-section measurements of $\mathrm{SO}_{2}$ at $160 \mathrm{~K}$ between 199 and 220 nm. J. Geophys. Res. 114, E06003. http://dx.doi.org/10.1029/2008JE003319.

Sandor, B.J., Clancy, R., T. , Moriarty-Schieven, G,. and F. P. Mills., 2010. Sulfur Chemistry in the Venus Mesosphere from $\mathrm{SO}_{2}$ and $\mathrm{SO}$ Microwave Spectra. Icarus, 208, p. 49-60.

Seiff, A., J. T. Shcofield, A. J. Kliore, F. W. Taylor and S. S. Limaye, 1985. Models of the structure of the atmosphere of Venus from the surface to $100 \mathrm{~km}$ altitude, Adv. Space Res., 5, pp. 3-58.

Snow, M., Mcclintock, W.E., Rottman, G., and T. N. Woods,2005. Solar-Stellar Irradiancce Comparison Experiment II (SOLSTICE II): Examination of the solar-stellar comparison technique. Sol Phys 230, p. 295-324, http://dx.doi.org./10.1007/s11207-0058763-3. 
Stark, G., Smith, P.L., Rufus, J., Thorne, A.P., Pickering, J.C., Cox, G., 1999. High resolution photoabsorption cross-section measurements of SO2 at $295 \mathrm{~K}$ between 198 and 220 nm. J. Geophys. Res. 104 (E7), 16585-16590.

Titov, D.V., Taylor, F.W., Svedhem, H., Ignatiev, N.I., Markiewicz, W.J., Piccioni, G., et al., 2008. Atmospheric structure and dynamics as the cause of ultraviolet markings in the clouds of Venus. Nature 456, p. 620-623.

Titov, D.V., Markiewicz, W.J., Ignatiev, N.I., Song, Li., Limaye, S.S., SanchezLavega, A., Hesemann, J., Almeida, M., Roatsch, Th., Matz, K.-D., Scholten, F., Crisp, D., Esposito, L.W., Hviid, S.F., Jaumann, R., Keller, H.U., Moissl, R2012. Morphology of the cloud tops as observed by the Venus Express Monitoring Camera Icarus 217, p. $682-701$.

von Zahn, U. and V. I. Moroz, 1985. Composition of the Venus Atmosphere below $100 \mathrm{~km}$ altitude, Adv. Space Res. 5, pp. 173-195.

Wilquet, V., Fedorova, A., Montmessin, F., Drummond, R., Mahieux, A., Vandaele, A.C., Villard, E., Korablev, O., Bertaux, J.L., 2009. Preliminary characterization of the upper haze by SPICAV/SOIR solar occultation in UV to mid-IR onboard Venus Express. J. Geophys. Res. 114, http://dx.doi.org/10.1029/2008JE003186.

Wilquet, V., Drummond, R., Mahieux, A., Robert, S., Vandaele, A.C., Bertaux, J.L., 2012. Optical extinction due to aerosols in the upper haze of Venus: Four years of SOIR/VEX observations from 2006 to 2010. Icarus 217, 875-881.

Yuk L. Yung,1 M. C. Liang, X. Jiang, R. L. Shia, C. Lee, B. Bézard, and E. Marcq, 2009. Evidence for carbonyl sulfide (OCS) conversion to CO in the lower atmosphere of Venus. J. Geophys. Res., 114, E00B34doi:10.1029/2008JE003094.

Zhang, X Liang, M.C., Montmessin, F., Bertaux, J.L., Parkinson, C., and Y. L. Yung,,2010. Photolysis of sulphuric acid as the source of sulphur oxides in the mesosphere of Venus, Nat Geosci 3:834-837.

Zhang, X., Liang, M. C.. Mills, F. P, Belyaev, Denis A., and Y. L. Yung., 2012. Sulfur chemistry in the middle atmosphere of Venus. Icarus, 217, p. 714-739.

\section{Figure Captions}

Figure 1. Adaption of a schematic presented in Mills et al. (2009) showing Venus' atmospheric structure between 44 and $120 \mathrm{~km}$. On the right axis, we highlight the altitude extent of Venus' lower troposphere, mesosphere and lower thermosphere; these 
demarcations reflect recent observations which have defined Venus' mesopause altitude to be $\sim 90 \mathrm{~km}$ (see Bertaux et al. 2007, Clancy et al. 2003). On the left axis we highlight the altitude range probed by observations made from nadir viewing at sub-mm (red), UV, and mid-IR (brown) wavelengths. While the sub-mm observations are known to be sensitive to the 70-100 km region (see Clancy et al. 2012), all known sub-mm observations of Venus are best fit by a 2-layer model where-in the exact magnitude of the abundance of the $\mathrm{SO}_{\mathrm{x}}$ species is only defined above $\sim 85 \pm 3 \mathrm{~km}$; thus the region between $70-85 \mathrm{~km}$ is represented as a dashed line (see Section 6.2 for details). UV observations made between 200-260 nm (indigo) and 260-390 nm (blue) are sensitive to the 68-78 km and 63-68 $\mathrm{km}$ altitude regions, respectively. In the mid-IR $(7-19 \mu \mathrm{m})$ the altitude sensitivity extends from 60 to $70 \mathrm{~km}$.

Figure 2. Adaptation of the plot presented in Fig. 3 of Marcq et al. (2013) showing the variability in Venus' average dayside Venus cloud top $(\mathrm{z} \sim 70-72 \mathrm{~km}) \mathrm{SO}_{2}$ volume mixing ratio (VMR) based on observations obtained between 1978 and 2011. The blue cross represents the average value derived from HST observations obtained between late December 2010 and late January 2011. The 1- $\sigma$ standard-deviation in the year-average values derived from SPICAV-UV nadir observations are plotted as solid red lines, the deviation in the observed $\mathrm{SO}_{2}$ VMR values is indicated by the dash red lines. Dayside average values plotted in black correspond to individual Pioneer Venus and Venera era observations made between 1978 and 1993, as well as the individual HST observation obtained in 1995 (Mills et al. 2007).

Figure 3. On 3 different dates we used the 0.1 " slit in conjunction with the CCD detector (0.051" pixels) and G230LB grating (170-306 nm) to obtain 40-60 km spatial resolution, $0.27 \mathrm{~nm}$ spectral resolution spectra that are sensitive to both the $\mathrm{SO}_{2}$ and $\mathrm{SO}$ gas absorption signatures. On the left we show an example of the long (52" x 0.1") HST/STIS slit (green line) intersecting the disk of Venus observable from the Earth. On the right we show an example of the spectra observed in each CCD pixel in the 2000-3040 $\AA$ (200$304 \mathrm{~nm}$ ) wavelength range; the band heads for the $\mathrm{SO}_{2} \mathrm{~B}-\mathrm{X}$ transitions $(180-240 \mathrm{~nm})$, $\mathrm{SO}_{2} \mathrm{~A}-\mathrm{X}$ transitions $(280-310 \mathrm{~nm})$, and SO B-X transitions $(190-240 \mathrm{~nm}$ ) are as indicated by the red, black and blue lines, respectively. The error in the data is as indicated by the orange error bars.

Figure 4. HST observations of Venus were obtained on 3 days, using the HST/STIS 0.1" long slit is oriented at a $45^{\circ}$ angle relative to Venus' equator. We show in the left, middle and right panels the illuminated region of Venus' disk observable from Earth on December 28, 2010; January 22, 2011; and January 27, 2011, respectively. On each schematic the orange line highlights the terminator longitude. On each date and in each exposure the slit is centered at the terminator longitude. Consequently, on each date data is obtained on Venus' dayside in the region extending from the morning terminator towards noon-however, during each of these observations the sub-solar longitude is either on the backside of Venus or in the forshortened region directly along the sunlit limb. Below each schematic we list the exact latitude and longitude at which the slit is centered for the two exposures taken on each date. 
Figure 5. In the top row we show details of the coordinated December 28, 2010 HST/STIS and VEx/SOIR observations, and in each panel on this row the SOIR occultation target latitude is highlighted. In the top-left panel we show the schematic of the HST/STIS slit paths (green lines) during OBS0 and OBS1; additionally the white line highlights the equator latitude and the orange line highlights the terminator longitude. In the top-middle panel we show the $365 \mathrm{~nm}$ VMC image taken at 0:26 UT December 28, 2010 coincident with OBS0, and just prior to the 2 UT SOIR occultation observations. The HST slit paths are overlayed in this image, and are observed to run diagonal to the latitude grid, as would be expected given the $45^{\circ}$ angle of the HST slit. In the top-right panel we show the projection of the $365 \mathrm{~nm}$ VMC image. In the bottom row we show details of the coordinated January 27, 2011 HST/STIS and VEx/VMC observations. In the bottom-left panel we show the schematic of the HST/STIS slit paths (green lines) during OBS4 and OBS5, as above the white line highlights the equator latitude and the orange line highlights the terminator longitude. In this row the $365 \mathrm{~nm}$ VMC image shown was obtained at 15:25 UT coincident with OBS4. In both the bottom-middle and bottom-right panels the HST slit paths for OBS4 and OBS5 are overlayed. In the middle panel we highlight the location of the morning terminator by a thick purple line, and indicate the latitude at which the HST/STIS intersects the terminator by a white asterisk for both OBS4 (orange diagonal path) and OBS5 (red diagonal path). In both the topright and bottom-right panels the absolute E. longitude value is provided on the projection of $365 \mathrm{~nm}$ VMC images in green; while the angular distance from the subsolar longitude $(\phi)$ is given in white; the maximum angular distance at which HST reliably detects the cloud top flux is for $\phi=\sim-75 \pm 5^{\circ}$. Although the absolute value of the E. longitude is different in the January 22, 2011 observations, the geometry is identical to the January 27, 2011 observations; thus, the same value of $\phi$ is encountered at each latitude observed on the two dates.

Figure 6. Comparison of the original (left panel) and updated (right panel) aerosol opacity model utilized in the radiative transfer code developed by Marcq et al. 2011.

Figure 7. Comparison of the fit $\mathrm{SO}_{2}$ column density as a function of the Solar Zenith Angle (SZA) in degrees (left) and latitude also referenced in degrees (right) on each of the HST dates of observation in 2010 and 2011. All the observations show a strong latitudinal dependence, though the gradient of the latitudinal variation observed on January 27, 2011 (bottom-right) is opposite to that observed on the previous dates. On December 28, 2010 (top panels) the $\mathrm{SO}_{2}$ gas density detections made near the equator (red-circle) were obtained at several SZAs, the $\mathrm{SO}_{2}$ gas density observed at $3 \mathrm{~N}$ at an SZA of $\sim 70^{\circ}$ ( $\mathrm{T}$, signifying near-terminator) is $\sim 2 \mathrm{x}$ greater than the gas density observed at $2 \mathrm{~N}$ at an SZA of $\sim 40^{\circ}$ (D, signifying dayside). Similarly, on January 22, 2011 (middle panels) the $\mathrm{SO}_{2}$ gas density detections made near $\sim 27 \mathrm{~S}$ (red-circle) were obtained at two different SZAs, in this case the gas density observed near the terminator at SZA $\sim 70^{\circ}(\mathrm{T})$ is $\sim 2 \mathrm{x}$ greater than that observed at $\sim 28 \mathrm{~S}$ at SZA $\sim 59^{\circ}$ (D). On January 27, (bottom panels) observations made in overlapping latitude regions, but at variable SZAs were all obtained at SZA $<60^{\circ}$, and in each case the detected gas densities were equivalent within the uncertainty of the fits (see Table IV). Although the $\mathrm{SO}_{2}$ gas density detected near the terminator (grey-circles) is higher than that observed at small SZA, in 
general the near-terminator gas density variation seems to be most strongly correlated with a steady increase in gas density with increasing latitude. On each date of observation there is strong overlap in the $\mathrm{SO}_{2}$ gas density detected closest to the equator at an SZA $<60^{\circ}$, implying that within the $\mathrm{SZA}<60^{\circ}$ region there is limited sensitivity in the equatorial gas density to the observed SZA. The only exception to this is the $\mathrm{SO}_{2}$ gas density observed at the equator on January 22, 2011 at SZA $\sim 17^{\circ}$ during OBS3 $(\mathrm{N}$, signifying near-noon) which is $\sim 2 \mathrm{x}$ smaller the other retrievals made between $\pm 15^{\circ}$ at SZAs of $20-50^{\circ}$ (see Table IV).

Figure 8. Variation in the fit $\mathrm{SO}_{2}$ (left) and $\mathrm{SO}$ (right) column density as a function of latitude on December 28, 2010 (top panels), January 22, 2011 (middle panels), and January 27, 2011 (bottom panels); where the color scheme and latitude units are the same as used in Figure 7.

Figure 9. Variation in the fit $\mathrm{SO}_{2}$ (left) and $\mathrm{SO}$ (right) column density as a function of Solar Zenith Angle (SZA) on December 28, 2010 (top panels), January 22, 2011 (middle panels), and January 27, 2011 (bottom panels); where the color scheme and SZA units are the same as used in Figure 7.

Figure 10. Projection of $365 \mathrm{~nm}(0.365 \mu \mathrm{m})$ VMC image obtained at 21:26 UT December 27, 2010, 2.5 hours before the 0-2 UT coincident HST and SOIR observing window, the longitude designations are relative to the sub-solar longitude, thus the morning terminator is on the far right edge of the right panel. (As in Fig. 4 the footprint of the HST slit during OBS 0 and OBS1 is overlayed onto the projection of the VMC image.) While there is clear evidence of contrast in the cloud brightness near the edge of the disk observed by VMC on the afternoon quadrant, in the same image the cloud brightness on the morning quadrant near the terminator is void of any significant cloud contrast features. Notably, the cloud brightness observed on the morning quadrant on January 22 and January 27, 2011 (see Fig. 4), was also fairly uniform.

Figure 11. Left panel: The calibrated intensity $\left(10^{5} \mathrm{~W} / \mathrm{m}^{3} / \mathrm{sr}\right)$ of the $0.365 \mu \mathrm{m} \mathrm{VMC}$ images directly below the footprint of the HST slits during the January 22, 2011 (OBS2 and OBS3) and January 27, 2011 (OBS4 and OBS5) observations is plotted as a function of latitude. Red markers highlight the latitude at which the HST slit intersected Venus' terminator longitude. Right panel: The footprint of the OBS4 and OBS5 HST slit paths is overlayed onto the projection of $365 \mathrm{~nm}$ VMC image obtained at 15:25 UT January 27, 2011, coincident with the 15:20-16:56 UT observing window of the January 27, 2011 HST observations. The exact same HST slit observing geometry was utilized on January 22 and January 27, 2011, thus OBS3 covers the same latitudes as OBS5 and OBS2 covers the same latitudes as OBS4. Although on both dates the cloud brightness on the morning quadrant was fairly uniform, the absolute brightness (intensity) of the cloud tops directly below the HST slit was lower north of 35S latitude on January 27 than on January 22; additionally, the gradient of the cloud top brightness changed between the two dates north of 20S latitude (left panel). In order to avoid the regions where there are data losses at the edge of the VMC image in the left panel we only plot the brightness data observed south of 11S latitude on January 27, 2011 and south of 16S latitude on January 22, 2011. 
Figure 12. Left: Ratios of the $0.245 \pm 0.004 \mu \mathrm{m}(2450-2650 \AA)$ cloud top reflectivity observed by HST at the equator on January 22, 2011 (black) and January 27, 2011(grey) relative to the reflectivity observed at the equator on December 28 , 2010, clearly show that the $0.245 \mu \mathrm{m}$ reflectivity had declined on each of the January 2011 observation dates. (note, the dashed line simply highlights the location of 1.0 on the vertical axis). Right: The relative $0.24 \mu \mathrm{m}$ brightness inferred from SPICAV-UV nadir observations obtained between 2006 and 2012 is plotted simultaneously while the low-latitude $\left( \pm 25^{\circ}\right)$ dayside $\mathrm{SO}_{2}$ gas column density retrieval ranges derived from these observations is indicated by the color code noted at the bottom of the panel. The SPICAV retrievals show an overall decline in the $\mathrm{SO}_{2}$ gas density from 2006 to late 2011. We outline the SPICAV retrievals obtained in late December 2010 and early January 2011 (orange box) and those obtained in March 2011 (grey box), for comparison to the 2010 and 2011 HST observations. On average the late December 2010/early January 2011 SPICAV retrievals were the 10-50 $\mu \mathrm{m}$-atm range, while the March 2011 SPICAV retrievals were 10x lower corresponding to $<5 \mu \mathrm{m}$-atm. These ranges are similar to the 2-12 $\mu \mathrm{m}$-atm and 0.4-1.9 $\mu \mathrm{m}$-atm values obtained by HST in 2010 and 2011, respectively. Likewise, the December 2010/early January 2011 the SPICAV retrieved $0.24 \mu \mathrm{m}$ Venus cloud top brightness levels were higher than the March 2011 values, duplicating the relative brightness levels observed by HST in similar time periods.

Figure 13. Both the HST and SPICAV-UV observations show instabilities in the $\mathrm{SO}_{2}$ latitudinal distribution. In the left panel we show the dayside $\mathrm{SO}_{2}$ gas column density retrieved by HST between late December 2010 and late January 2011 (see Table I), on each date the observed $\mathrm{SO}_{2}$ density is strongly overlapping close to the equator, and on each date the $\mathrm{SO}_{2}$ density has a strong latitudinal variance, though the latitude of maximum $\mathrm{SO}_{2}$ density is variable. In the right panel we show the $\mathrm{SO}_{2}$ latitude pattern observed by VEx/SPICAV between 2006-2008, the dominant pattern (highlighted in brown) is that the retrieved $\mathrm{SO}_{2}$ gas column density increases from high latitude towards low latitude, and are strongly overlapping between $30 \mathrm{~N}$ and $20 \mathrm{~S}$ latitude. However, the VEx retrieved densities were also observed to decrease northward of 30S latitude (outlined in grey), while at other times the complete reverse trend was observed between $20 \mathrm{~S}$ and $20 \mathrm{~N}$ latitude (pink bars). Note in the right panel, color represents the Venus express orbit number, see Marcq et al. 2011.

Figure 14. The $\mathrm{SO}_{2}$ number density profiles best fit to the HST observations obtained on December 28, 2010 (far left), Jan 22, 2011 (middle) and Jan 27, 2011 (far right), and compared to the $5.3 \times 10^{10} \mathrm{~cm}^{-3} \mathrm{SO}_{2}$ number density (orange $\mathrm{x}$ ) inferred from SOIR profiles obtained coincidentally with HST on December 28, 2010 on the morning terminator $\left(\mathrm{SZA}=90^{\circ}\right)$ at a target latitude of $31 \mathrm{~S}$, with a vertical resolution extending from $74-81 \mathrm{~km}$ as indicated by the orange line. In the far left panel the green line is the average value profile fit at $\mathrm{SZA}=70^{\circ}$, at a latitude (LAT) of $\sim 16 \pm 3 \mathrm{~S}$. In each panel the plotted profiles replicate the $\mathrm{SO}_{2}$ column density fit at the $\tau=1$ altitude for the aerosols; in fitting the observations the aerosol opacity is a free parameter, and the exact altitude at which $\tau=1$ is obtained depends on the assumed aerosol opacity. In each panel we show the profile associated with the column densities fit at the min and max $\tau=1$ (grey dashed) 
altitude encountered within the uncertainty of the minimum chi-square fit of the data, and the average of these profiles (green solid). In each panel the average number density derived from the HST profiles at $\mathrm{SZA}=70^{\circ}$ is presented as a green asterisk, and corresponds to values of $5.36 \times 10^{10}, 1.0 \times 10^{10}$, and $0.49 \times 10^{10} \mathrm{~cm}^{-3}$ on December 28, 2010, Jan 22, 2011 and Jan 27, 2011 respectively. Note that as the fit column densities decrease distinction between the min and max profiles becomes less visible on the log scale; on December 28, 2010 the $\tau=1$ altitude at $\mathrm{SZA}=70^{\circ}$ was $=75.1 \pm 2.3 \mathrm{~km}$, on the other two dates the altitude uncertainty at $\mathrm{SZA}=70^{\circ}$ was $\sim 0.5 \mathrm{~km}$.

Figure 15. Schematics from Petrass 2013, which represent the basic components leading to the $\mathrm{SO}_{2} \mathrm{VMR}$ vertical profiles predicted from several recent photochemical models (e.g., Kransopolsky 2012, Mills 1998, Mills et al. 2007, Petrass 2013, Yung et al. 2009). The schematic on the left highlights that the availability of sulfur bearing molecules that react with $\mathrm{SO}_{2}$ producing species (such as $\mathrm{O}$ and $\mathrm{S}$ ) increases the rate at which $\mathrm{SO}_{2}$ decreases as a function of altitude-leading to exponential decrease of $\mathrm{SO}_{2}$ above $\sim 60$ $\mathrm{km}$, before becoming near constant at high altitude. The schematic on the right highlights that if there is a set of reactions that can lead to a quick destruction and production of $\mathrm{SO}_{2}$, producing a net increase of $\mathrm{SO}_{2}$ in the 80-90 km region, then an inversion layer will be formed near $80 \mathrm{~km}$. The average dayside low-latitude vertical $\mathrm{SO}_{2}$ mixing ratio profile inferred from the combined HST and JCMT data maps well to the prediction that the $\mathrm{SO}_{2}$ VMR profile is near constant above $86 \mathrm{~km}$, and exponentially decreasing below $\sim 80 \mathrm{~km}$ (left panel). However there is a gap between the $74-81 \mathrm{~km}$ altitude sensitivity of the HST retrievals and the $85 \pm 1 \mathrm{~km}$ cut-off altitude inferred from the 2-layer model used to fit the sub-mm data. Thus, the small inversion layer depicted in the right panel may yet exist between $81-86 \mathrm{~km}$.

Figure 16. Comparison of the $\mathrm{SO}_{2}$ (left) and $\mathrm{SO}$ (right) volume mixing ratios derived from the HST UV and JCMT sub-mm observations in the $74-81 \mathrm{~km}$ and $86-100 \mathrm{~km}$ altitude ranges, respectively.

Figure 17. The $\mathrm{SO}_{2} / \mathrm{SO}$ abundance ratios derived from the coordinated HST UV (red) and JCMT sub-mm (orange) dayside observations in the 74-81 km and 85-100 km altitude ranges, respectively, are compared to the ratio values predicted by several photochemical models; as well as the 2004-2008 JCMT (black) day and nightside observations. The 74-100 km SO$/ 2$ profile inferred from the combination of these observations is reasonably consistent with the Mills et al. predictions for the dayside $\mathrm{SO}_{2} / \mathrm{SO}$ gas abundance ratio. The "Mills et al." model referenced in this figure was first presented in Sandor et al. 2010, and is a simplification of the Mills and Allen (2007) and Mills et al. (2007) model to include only the 20 reactions responsible for $95 \%$ of the modeled production and loss of $\mathrm{SO}_{2}, \mathrm{SO}$, and $\mathrm{SOx}\left(=\mathrm{SO}_{2}+\mathrm{SO}\right)$ in Venus' atmosphere. In these models the day and nightside behavior is derived based on the global average solar flux, which is estimated as being equivalent to the solar flux at $\mathrm{LAT}=45^{\circ}$ divided by 2 ; the nightside behavior is specifically determined by excluding all photo-dissociation reactions. 
Figure 18. Predictions of the vertical $\mathrm{SO}_{2}$ number density (left panel) and $\mathrm{SO}_{2}$ volume mixing ratio (VMR) profiles derived from 1-d photochemical modeling assuming photochemical steady-state and using the solar flux expected at SZA $=45^{\circ}$ and $70^{\circ}$ on the equator on December 28, 2010. In the left panel we also overlay the average of the modeled $\mathrm{SO}_{2}$ number density at $\mathrm{SZA}=45^{\circ}$ (plus symbol) and $70^{\circ}$ (square symbol) in the 74-81 km region consistent with the vertical resolution of Venus Express occultation observations obtained on December 28, 2010, as well as the average dayside (red triangle) and near-terminator (orange asterisk) values expected at $16 \pm 3 \mathrm{~S}$ latitude based on trends inferred from the HST observations obtained on that date. In the right panel, in addition to the vertical profiles derived from the diurnally variant photochemical scheme, we also overlay the Model A (grey-solid) and Model C (grey-dashed) $\mathrm{SO}_{2} \mathrm{VMR}$ profiles derived by Zhang et al. (2010), where the latter includes the chemistry needed to induce an $\mathrm{SO}_{2}$ inversion layer above $75 \mathrm{~km}$. Note that the overall magnitude of the $\mathrm{SO}_{2} \mathrm{VMR}$ derived from the Zhang et al. 2010 model is lower than that produced from the diurnally variant photochemical; this seems to result from the fact that the assumed $\mathrm{H}_{2} \mathrm{SO}_{4}$ production rate (resulting in a net loss of $\mathrm{SO}_{2}$ gas) in the two models is different.

Figure 19. The relative timescales for photochemical loss of $\mathrm{SO}_{2}$ and transport in the mesosphere and lower thermosphere at 0 to $70^{\circ} \mathrm{SZA}$ are calculated and plotted in contours of the base-10 logarithm of the ratio of the photochemical loss timescale to either the horizontal (top-panels) or vertical (bottom-panels) transport timescale. In the top bottom panels, the timescale for vertical eddy mixing is calculated from $\tau_{\text {vert }}=$ (density scale height) ${ }^{2}$ / (eddy diffusion coefficient), assuming the vertical profiles for density scale height and eddy diffusion coefficient are the same at all SZA; and in the bottom top panels the timescale for zonal transport is calculated from $\tau_{\text {zonal }}=(1000 \mathrm{~km}) /$ (wind speed), assuming a zonal wind speed of $90 \mathrm{~ms}^{-1}$ (Khatuntsev et al. 2013) at all altitudes and SZA; thus the $1000 \mathrm{~km}$ value corresponds to a $\sim 3 \mathrm{hr}$ zonal transport lengthscale. The calculations are completed relative to the timescale for both the photochemical oxidation of $\mathrm{SO}_{2}$ to $\mathrm{SO}_{3}$ (right panels) and the timescale for photodissociation of $\mathrm{SO}_{2}$ to $\mathrm{SO}$ (left panels). Regions where the base-10 logarithm of the ratio is $\leq 0$ represent where the photochemistry timescale is shorter than/equivalent to the transport timescale, and are highlighted in color. In the right panels the colored regions highlight where $\mathrm{SO}_{2}$ and $\mathrm{SO}$ are expected to be in photochemical equilibrium with each other. In the bottom-right panel, the colored region highlights where $\mathrm{SO}_{3}$ formation is highest, these regions also correspond to where the formation of the $\mathrm{H}_{2} \mathrm{SO}_{4}$ aerosol/gas species is most likely. Conversely in the un-colored regions, where the ratio is positive, the transport timescale is shorter than the photochemical time scale. It is the relative timescales for photo-dissociation of $\mathrm{SO}_{2}$ and transport (right panels) that drive the relationships presented in Figure 18. 
TABLE I

\begin{tabular}{|c|c|c|c|c|c|c|c|c|c|c|c|}
\hline \\
\hline $\begin{array}{l}\text { OBSERVATION } \\
\text { NUMBER }\end{array}$ & $\begin{array}{c}\text { HST } \\
\text { filename }\end{array}$ & $\begin{array}{c}\text { date } \\
\text { (yyyy-mm-dd) }\end{array}$ & $\begin{array}{c}\text { target } \\
\text { latitude }\end{array}$ & $\begin{array}{c}\text { terminator } \\
\text { longitude }\end{array}$ & $\begin{array}{c}\begin{array}{c}\text { sub- } \\
\text { earth } \\
\text { longitude }\end{array} \\
\end{array}$ & $\begin{array}{c}\text { Planet } \\
\text { radius } \\
\text { (arcsec) }\end{array}$ & $\begin{array}{c}\% \\
\text { illuminated }\end{array}$ & $\begin{array}{c}\text { mid-exposure } \\
\text { time } \\
\text { (UT) } \\
\end{array}$ & $\begin{array}{c}\text { exposure } \\
\text { length } \\
\text { (s) }\end{array}$ & grating & $\begin{array}{c}\text { Visit } \\
\text { number }\end{array}$ \\
\hline 0 & obo0a1010_x2d.fits & $2010-12-28$ & $15 \mathrm{~S}$ & $65 \mathrm{E}$ & $72.4 \mathrm{E}$ & 14.3 & $44 \%$ & $00: 21: 54$ & 153 & G230LB & V01 \\
\hline 1 & obo0c1010_x2d.fits & $2010-12-28$ & $32 \mathrm{~s}$ & $65 \mathrm{E}$ & $72.5 \mathrm{E}$ & 14.3 & $44 \%$ & 01:58:21 & 153 & G230LB & V01 \\
\hline 2 & obo0a2010_x2d.fits & 2011-01-22 & $45 \mathrm{~S}$ & $145 \mathrm{E}$ & $136.6 \mathrm{E}$ & 10.7 & $57 \%$ & $17: 03: 55$ & 58 & G230LB & V02 \\
\hline 3 & obo0c2010_x2d.fits & 2011-01-22 & $65 \mathrm{~S}$ & $145 \mathrm{E}$ & $136.8 \mathrm{E}$ & 10.7 & $57 \%$ & 18:39:38 & 58 & G230LB & V02 \\
\hline 4 & obo0a3010 sx2.fits & 2011-01-27 & $45 \mathrm{~S}$ & $160 \mathrm{E}$ & $149.4 \mathrm{E}$ & 10.2 & $59 \%$ & $15: 20: 20$ & 116 & G230LB & V03 \\
\hline 5 & obo0c3010 sx2.fits & $2011-01-27$ & $65 \mathrm{~S}$ & $160 \mathrm{E}$ & $149.5 \mathrm{E}$ & 10.2 & $59 \%$ & $16: 56: 13$ & 116 & G230LB & V03 \\
\hline
\end{tabular}


TABLE II

\begin{tabular}{|c|c|c|c|c|c|c|c|c|c|}
\hline & $\begin{array}{c}\text { HST } \\
\text { observation } \\
\text { Time } \\
\text { (UT) }\end{array}$ & $\begin{array}{c}\text { HST } \\
\text { Dayside } \\
\text { latitude+ } \\
\text { longitudes }\end{array}$ & $\begin{array}{l}\text { VEx } \\
\text { Orbit }\end{array}$ & $\begin{array}{c}\text { SPICAV-IR } \\
\text { obs. \# |time (UT) }\end{array}$ & $\begin{array}{l}\text { SPICAV-IR } \\
\text { cloud height } \\
\text { |assumed } \lambda\end{array}$ & $\begin{array}{c}\text { VMC }^{\dagger \dagger} \\
\text { obs. \# | time (UT) }\end{array}$ & $\begin{array}{c}\text { SOIR } \\
\text { obs. \# |time } \\
\text { (UT) }\end{array}$ & $\begin{array}{c}\text { SOIR altitude } \\
\text { sensitivity }\end{array}$ & $\begin{array}{c}\text { Ground based } \\
\text { altitude } \\
\text { sensitivity } \\
\text { sub-mm } \lambda\end{array}$ \\
\hline Dec 28, 2010 & $00: 21: 54$ & $\begin{array}{l}\text { Lat: } 15 S 30 N \\
\text { Lon: } 64-326 E\end{array}$ & 1712 & No observations & & $\begin{array}{l}\text { Pre-Occultation } \\
\text { observation/only } \\
\text { crescent observed }\end{array}$ & & N/A & \multirow{2}{*}{$\begin{array}{c}\text { Dayside }^{\S} \\
\text { z>84 km } \\
\text { (observation time } \\
\text { 15:20-18:10 UT) }\end{array}$} \\
\hline Dec 28, 2010 & 01:58:21 & $\begin{array}{l}\text { Lat: } 32 \mathrm{~S} 20 \mathrm{~N} \\
\text { Lon: } 64-326 \mathrm{E}\end{array}$ & 1712 & $\begin{array}{c}\text { \#04| 2:42-3:01 } \\
\text { Lat:30N } \\
\text { Lon:310E }\end{array}$ & $\begin{array}{c}\dagger 70.5-71 \mathrm{~km} \mid \\
\lambda \sim 1.45 \mu \mathrm{m}\end{array}$ & 40|02:31:46 & $\begin{array}{c}\# 01 \mid \\
01: 48: 54 \\
\text { Lat:31S } \\
\text { Lon:63E } \\
\end{array}$ & 73-100 km & \\
\hline Dec 29, 2010 & & & 1713 & $\begin{array}{c}\# 03 \mid 2: 43-2: 57 \\
\text { Lat:41N-10S } \\
\text { Lon:310E } \\
\end{array}$ & $\begin{array}{c}70.5-72.5 \mathrm{~km} \mid \\
\lambda \sim 1.45 \mu \mathrm{m}\end{array}$ & & & & \\
\hline Jan 22, 2011 & 17:03:55 & $\begin{array}{l}\text { Lat: } 45 S 20 \mathrm{~N} \\
\text { Lon: } 144-46 \mathrm{E}\end{array}$ & 1738 & No observations & & $\begin{array}{l}28 \mid 16: 56: 06 \\
32 \mid 17: 11: 06 \\
36 \mid 17: 26: 06 \\
\end{array}$ & $\begin{array}{c}\text { No } \\
\text { observations }\end{array}$ & N/A & \multirow{2}{*}{$\begin{array}{c}\text { Dayside } \\
\mathrm{z}>84 \mathrm{~km} \\
\text { (observation time } \\
\text { 18:10-20:00 UT) }\end{array}$} \\
\hline Jan 22, 2011 & 18:39:38 & $\begin{array}{l}\text { Lat: } 65 \mathrm{~S} 10 \mathrm{~N} \\
\text { Lon: } 144-46 \mathrm{E}\end{array}$ & 1738 & $\begin{array}{l}\# 02 \mid 18: 51-19: 10 \\
\text { No data - low Sun }\end{array}$ & & $\begin{array}{l}\text { 52|18:26:06 } \\
56 \mid 18: 41: 06 \\
60 \mid 18: 56: 06\end{array}$ & $\begin{array}{c}\text { No } \\
\text { observations }\end{array}$ & N/A & \\
\hline Jan 23, 2011 & & & & & & & & & $\begin{array}{c}\text { Dayside } \\
\mathrm{z}>84 \mathrm{~km} \\
19: 40-22: 30 \mathrm{UT}\end{array}$ \\
\hline Jan 27, 2011 & $15: 20: 20$ & $\begin{array}{l}\text { Lat: } 45 \mathrm{~S} 20 \mathrm{~N} \\
\text { Lon: } 159-59 \mathrm{E}\end{array}$ & 1743 & No observations & & $\begin{array}{l}04 \mid 15: 10: 32 \\
08 \mid 15: 25: 32 \\
12 \mid 15: 40: 32\end{array}$ & $\begin{array}{c}\text { No } \\
\text { observations }\end{array}$ & N/A & No observations \\
\hline Jan 27, 2011 & 16:56:13 & $\begin{array}{l}\text { Lat: } 65 \mathrm{~S} 10 \mathrm{~N} \\
\text { Lon: } 159-59 \mathrm{E}\end{array}$ & 1743 & No observations & & $\begin{array}{l}28 \mid 16: 40: 32 \\
32 \mid 16: 55: 32 \\
36 \mid 17: 10: 32 \\
\end{array}$ & $\begin{array}{c}\text { No } \\
\text { observations }\end{array}$ & N/A & No observations \\
\hline Jan 28, 2011 & & & & & & & & & $\begin{array}{c}\text { Dayside } \\
\mathrm{z}>84 \mathrm{~km} \\
\text { 16:30-21:30 UT }\end{array}$ \\
\hline Jan 30, 2011 & & & & & & & & & $\begin{array}{c}\text { Dayside } \\
\text { z }>84 \mathrm{~km} \\
15: 30-17: 20 \text { UT }\end{array}$ \\
\hline
\end{tabular}

\section{HST/STIS observing dates highlighted in pink}

†cloud top altitude inferred assuming aerosol scale height of 4 ; the $1.45 \mu \mathrm{m}$ cloud top values are the average of the $1.48 \mu \mathrm{m}$ and $1.43 \mu \mathrm{m}$ values; the $0.36 \mu \mathrm{m}$ cloud top is typically $2 \mathrm{~km}$ higher than $1.51 \mu \mathrm{m}$ cloud top; thus, for our UV observations we adopt the value $\sim 2 \mathrm{~km}$ higher than $1.48 \mu \mathrm{m}$ value

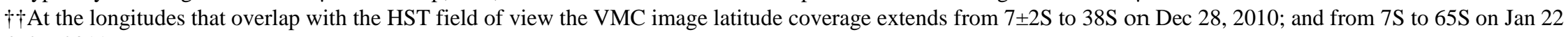
\& 27, 2011

$\S$ sub-mm observations are disk integrated, dayside observations 
TABLE IV

\begin{tabular}{|c|c|c|c|c|c|c|c|c|c|c|c|c|c|c|}
\hline$\phi$ & $\begin{array}{c}\text { OBS. } \\
\#\end{array}$ & 茟 & $\underset{\mathbb{N}}{\mathbb{N}}$ & \multicolumn{2}{|c|}{$\begin{array}{l}\text { LOCAL } \\
\text { TIME } \\
\text { (hr) }\end{array}$} & \multicolumn{2}{|c|}{$\begin{array}{c}\text { Total } \\
\text { aerosol } \\
\tau=1 \\
\text { ALTITUDE }\end{array}$} & $\begin{array}{l}\mathrm{NCO}_{2} \\
\mathrm{x} 10^{7} \\
(\mu \mathrm{m} \text {-atm) }\end{array}$ & $\begin{array}{c}\mathrm{SO}_{2} \mathrm{VMR} \\
\quad(\mathrm{ppb})\end{array}$ & $\begin{array}{c}\text { SO VMR } \\
(\mathrm{ppb})\end{array}$ & $\begin{array}{l}\mathrm{NSO}_{2} \\
(\mu \mathrm{m} \text {-atm) }\end{array}$ & $\begin{array}{c}N S O \\
(\mu \mathrm{m}-\mathrm{atm})\end{array}$ & $\begin{array}{c}\text { Mode } 1 \\
\text { aerosol } \\
\text { opacity }(\tau)\end{array}$ & $\begin{array}{l}\text { Bright. } \\
\text { factor }\end{array}$ \\
\hline-15 & 0 & \multicolumn{13}{|c|}{ no data (back side of disk)-- no data (back side of disk) --no data (back side of disk) } \\
\hline-20 & 0 & 28 & 33 & \multicolumn{2}{|c|}{10.7} & \multicolumn{2}{|c|}{$75.2 \pm 0.5$} & $7.30 \pm 0.59$ & $30.6 \pm 0.11$ & $3.38 \pm 1.23$ & $2.2 \pm 0.2$ & $0.26 \pm 0.1$ & $2.05 \pm 0.45$ & $0.80 \pm 0.04$ \\
\hline-25 & 0 & 27 & 36 & \multicolumn{2}{|c|}{10.3} & \multicolumn{2}{|c|}{$74.7 \pm 1.0$} & $8.00 \pm 1.2$ & $41.6 \pm 1.22$ & $4.49 \pm 1.66$ & $3.2 \pm 0.4$ & $0.34 \pm 0.1$ & $1.90 \pm 0.60$ & $0.81 \pm 0.06$ \\
\hline-30 & 0 & 25 & 38 & \multicolumn{2}{|c|}{10.0} & \multicolumn{2}{|c|}{$75.4 \pm 1.1$} & $6.9 \pm 0.99$ & $53.8 \pm 2.44$ & $4.87 \pm 1.27$ & $3.6 \pm 0.2$ & $0.35 \pm 0.1$ & $2.05 \pm 0.45$ & $0.85 \pm 0.04$ \\
\hline-40 & 0 & \multicolumn{13}{|c|}{ no data (instrument fiducial) } \\
\hline-45 & 0 & 19 & 48 & \multicolumn{2}{|c|}{9.0} & \multicolumn{2}{|c|}{$74.9 \pm 1.5$} & $7.61 \pm 1.6$ & $67.3 \pm 3.06$ & $6.84 \pm 2.13$ & $5.0 \pm 1.0$ & $0.50 \pm 0.2$ & $1.90 \pm 0.60$ & $0.88 \pm 0.06$ \\
\hline-50 & 0 & 18 & 51 & \multicolumn{2}{|c|}{8.7} & \multicolumn{2}{|c|}{$75.2 \pm 0.5$} & $7.30 \pm 0.59$ & $62.7 \pm 1.50$ & $5.29 \pm 2.68$ & $4.4 \pm 0.5$ & $0.36 \pm 0.2$ & $2.05 \pm 0.45$ & $0.81 \pm 0.04$ \\
\hline-55 & 0 & 15 & 55 & \multicolumn{2}{|c|}{8.4} & \multicolumn{2}{|c|}{$74.7 \pm 1.0$} & $8.00 \pm 1.2$ & $90.8 \pm 1.04$ & $9.43 \pm 3.15$ & $7.0 \pm 1.0$ & $0.72 \pm 0.2$ & 0.60 & $0.92 \pm 0.06$ \\
\hline-60 & 0 & 11 & 60 & \multicolumn{2}{|c|}{8.0} & \multicolumn{2}{|c|}{$75.4 \pm 2.0$} & $7.05 \pm 2.2$ & $114 \pm 13.8$ & $9.08 \pm 2.01$ & $8.0 \pm 2.0$ & $0.68 \pm 0.3$ & .75 & 0.7 \\
\hline-70 & 0 & 3 & 69 & \multicolumn{2}{|c|}{7.3} & \multicolumn{2}{|c|}{$74.2 \pm 2.2$} & $8.87 \pm 2.9$ & $198 \pm 5.99$ & $16.3 \pm 6.69$ & $17.2 \pm 6.2$ & $1.24 \pm 0.5$ & $1.90 \pm 0.89$ & $0.86 \pm 0.07$ \\
\hline \multicolumn{3}{|l|}{ 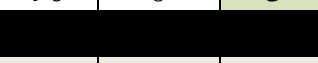 } & \multicolumn{12}{|c|}{ no data (back side of disk)-- no data (back side of disk) --no data (back side of disk) } \\
\hline-20 & $\frac{1}{1}$ & \multicolumn{13}{|c|}{ no data (forshortened sunlit limb) --no data (forshortened sunlit limb)-- no data (forshortened sunlit limb) } \\
\hline-25 & 1 & 7 & 26 & \multicolumn{2}{|c|}{10.3} & \multicolumn{2}{|c|}{$74.7 \pm 1.0$} & $8.00 \pm 1.2$ & $77.5 \pm 0.97$ & $8.90 \pm 3.41$ & $6.0 \pm 1.0$ & $0.67 \pm 0.3$ & $2.05 \pm 0.75$ & $0.72 \pm 0.05$ \\
\hline-30 & 1 & 5 & 32 & \multicolumn{2}{|c|}{9.9} & \multicolumn{2}{|c|}{$74.8 \pm 1.6$} & $7.61 \pm 1.6$ & $103 \pm 2.20$ & $7.70 \pm 1.38$ & $7.5 \pm 1.5$ & $0.59 \pm 0.2$ & $2.05 \pm 0.75$ & $0.78 \pm 0.05$ \\
\hline-40 & 1 & 2 & 40 & \multicolumn{2}{|c|}{9.3} & \multicolumn{2}{|c|}{$74.8 \pm 2.2$} & $7.26 \pm 2.0$ & $106 \pm 5.08$ & $9.23 \pm 3.08$ & $7.3 \pm 1.7$ & $0.70 \pm 0.2$ & $2.05 \pm 0.75$ & $0.75 \pm 0.05$ \\
\hline-45 & 1 & -1 & 46 & \multicolumn{2}{|c|}{8.9} & & $3 \pm 2.2$ & $7.26 \pm 2.0$ & $141 \pm 6.78$ & $9.17 \pm 3.52$ & $9.8 \pm 2.3$ & $0.70 \pm 0.3$ & $2.05 \pm 0.75$ & $0.81 \pm 0.05$ \\
\hline-50 & 1 & -3 & 49 & & & & $3 \pm 2.1$ & $7.61 \pm 1.6$ & $132 \pm 8.37$ & $7.46 \pm 4.60$ & $10 \pm 2.0$ & $0.67 \pm 0.4$ & $2.05 \pm 0.75$ & $0.78 \pm 0.05$ \\
\hline-60 & 1 & -9 & 60 & & & & \pm 1.3 & $8.00 \pm 1.2$ & $106 \pm 5.41$ & $10.5 \pm 4.77$ & $8.5 \pm 1.6$ & $0.87 \pm 0.4$ & $1.90 \pm 0.60$ & $0.82 \pm 0.06$ \\
\hline-70 & 1 & -16 & 70 & & & & \pm 2.3 & $8.32 \pm 3.5$ & $76.5 \pm 8.91$ & $8.74 \pm 3.50$ & $6.4 \pm 2.3$ & $0.70 \pm 0.4$ & $1.90 \pm 0.89$ & $0.95 \pm 0.07$ \\
\hline & $/ 28 / 20$ & & & $v_{2}$ & $\mathrm{In}^{-\mathrm{at}}$ & & NSO & $(\mu \mathrm{m}-\mathrm{atm})$ & & ${ }_{2}$ VMR (ppb) & & & SO VMR (pF & \\
\hline OBS & $\begin{array}{c}\text { RVAT } \\
0+1\end{array}$ & DNS & & & & & 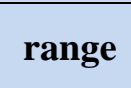 & Average & & average & & & Average & \\
\hline da & ide de & & 6.0 & & 5.7 & 1.0 & $0.5 \pm 0.2$ & $0.5 \pm 0.2$ & & $78.0 \pm 2.8$ & & & $6.6 \pm 2.5$ & \\
\hline term & nator d & isity & & & 10.0 & \pm 3.0 & $1.0 \pm 0.3$ & $0.9 \pm 0.4$ & & $124 \pm 8$ & & & $11.1 \pm 3.9$ & \\
\hline
\end{tabular}


TABLE IV (cont'd)

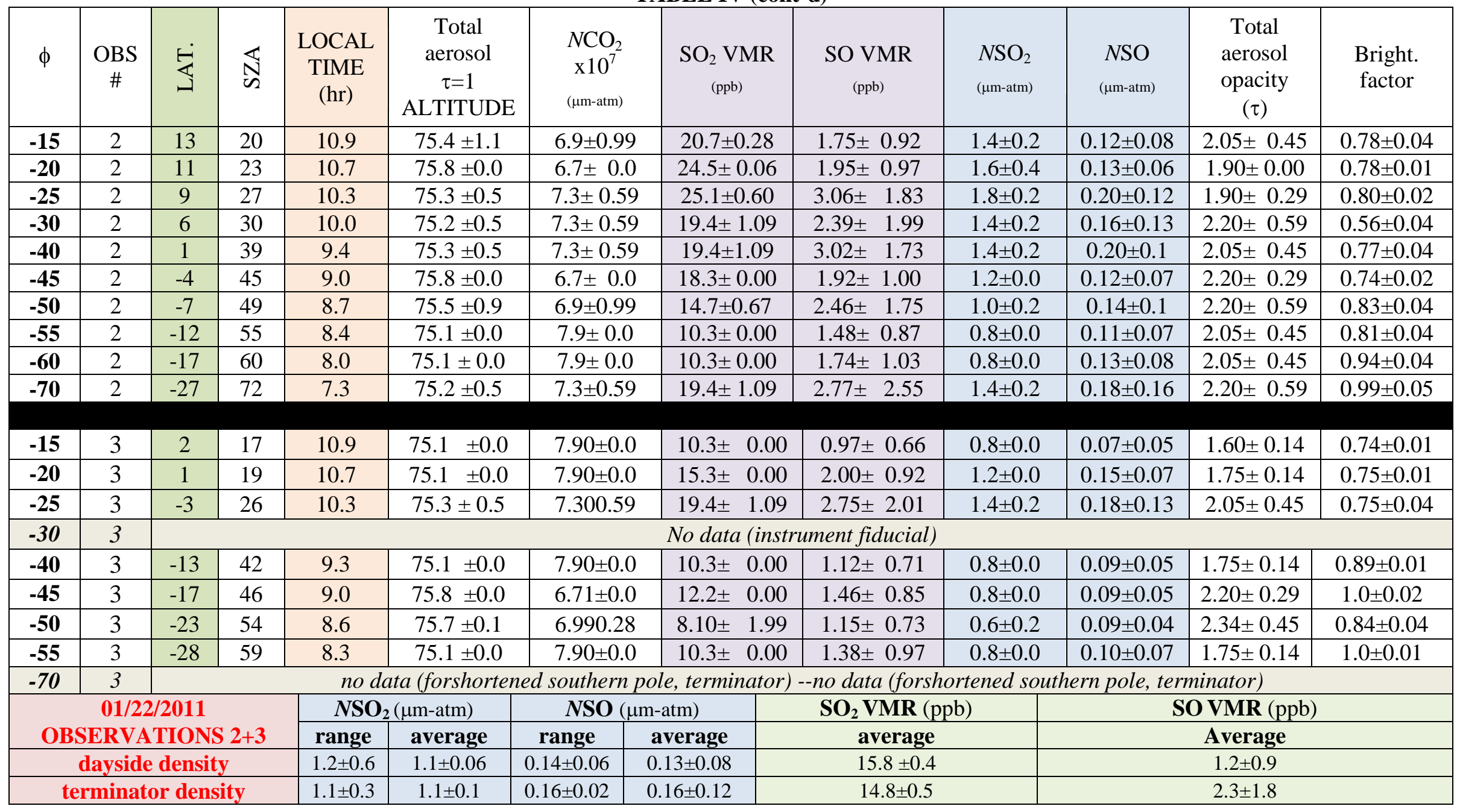


TABLE IV (cont'd)

\begin{tabular}{|c|c|c|c|c|c|c|c|c|c|c|c|c|}
\hline$\phi$ & $\begin{array}{c}\text { OBS } \\
\#\end{array}$ & 岕 & $\underset{\mathbb{N}}{\text { w }}$ & $\begin{array}{c}\text { LOCAL } \\
\text { TIME } \\
\text { (hr) }\end{array}$ & $\begin{array}{c}\text { Total } \\
\text { aerosol } \\
\tau=1 \\
\text { ALTITUDE }\end{array}$ & $\begin{array}{c}\mathrm{NCO}_{2} \\
\text { x10 } \\
\text { ( } \mu \mathrm{m} \text {-atm) }\end{array}$ & $\begin{array}{c}\mathrm{SO}_{2} \mathrm{VMR} \\
\quad(\mathrm{ppb})\end{array}$ & $\begin{array}{c}\text { SO VMR } \\
(\mathrm{ppb})\end{array}$ & $\begin{array}{l}\mathrm{NSO}_{2} \\
(\mu \mathrm{m}-\mathrm{atm})\end{array}$ & $\begin{array}{c}\text { NSO } \\
(\mu \mathrm{m}-\mathrm{atm})\end{array}$ & $\begin{array}{c}\text { Total } \\
\text { aerosol } \\
\text { opacity }(\tau)\end{array}$ & $\begin{array}{l}\text { Bright. } \\
\text { factor }\end{array}$ \\
\hline-15 & 4 & 17 & 24 & 10.9 & $75.1 \pm 0.0$ & $7.90 \pm 0.0$ & $10.3 \pm \quad 0.00$ & $1.12 \pm 0.61$ & $0.8 \pm 0.0$ & $0.09 \pm 0.05$ & $1.75 \pm 0.14$ & $0.80 \pm 0.01$ \\
\hline-20 & 4 & 16 & 25 & 10.7 & $76.8 \pm 0.6$ & $5.36 \pm 0.55$ & $7.38 \pm 0.34$ & $1.32 \pm 0.83$ & $0.4 \pm 0.02$ & $0.07 \pm 0.03$ & $2.50 \pm 0.29$ & $0.74 \pm 0.02$ \\
\hline-25 & 4 & 13 & 28 & 10.3 & $76.4 \pm 0.0$ & $5.92 \pm 0.0$ & $7.02 \pm 0.00$ & $1.32 \pm 0.84$ & $0.4 \pm 0.0$ & $0.09 \pm 0.03$ & $2.50 \pm 0.29$ & $0.73 \pm 0.02$ \\
\hline-30 & 4 & 11 & 32 & 10.0 & $75.4 \pm 1.1$ & $6.9 \pm 0.99$ & $8.65 \pm 1.62$ & $1.18 \pm 0.97$ & $0.6 \pm 0.2$ & $0.07 \pm 0.02$ & $2.50 \pm 0.59$ & $0.70 \pm 0.04$ \\
\hline-40 & 4 & 4 & 39 & 9.4 & $76.4 \pm 0.0$ & $5.92 \pm 0.0$ & $6.67 \pm 0.35$ & $1.19 \pm 0.76$ & $0.4 \pm 0.02$ & $0.07 \pm 0.04$ & $2.50 \pm 0.29$ & $0.71 \pm 0.02$ \\
\hline-50 & 4 & -4 & 49 & 8.7 & $76.7 \pm 0.7$ & $5.36 \pm 0.55$ & $7.38 \pm 0.34$ & $1.22 \pm 0.74$ & $0.4 \pm 0.02$ & $0.07 \pm 0.04$ & $2.50 \pm 0.29$ & $0.70 \pm 0.02$ \\
\hline-60 & 4 & -14 & 60 & 8.0 & $75.9 \pm 0.5$ & $6.3 \pm 0.39$ & $6.22 \pm 0.10$ & $0.94 \pm 0.76$ & $0.4 \pm 0.02$ & $0.06 \pm 0.05$ & $2.34 \pm 0.45$ & $0.73 \pm 0.04$ \\
\hline-70 & 4 & -25 & 71 & 7.4 & $74.6 \pm 0.5$ & $8.60 \pm 0.69$ & $11.8 \pm 1.58$ & $1.94 \pm 1.22$ & $1.0 \pm 0.2$ & $0.15 \pm 0.09$ & $2.05 \pm 0.75$ & $0.87 \pm 0.05$ \\
\hline-15 & 5 & 1 & 17 & 10.9 & $76.4 \pm 0.0$ & $5.92 \pm 0.0$ & $7.02 \pm 0.00$ & $1.32 \pm 0.84$ & $0.4 \pm 0.0$ & $0.09 \pm 0.03$ & $2.50 \pm 0.29$ & $0.73 \pm 0.02$ \\
\hline-20 & 5 & 0 & 19 & 10.7 & $76.4 \pm 0.0$ & $5.92 \pm 0.0$ & $7.02 \pm 0.00$ & $1.25 \pm 0.77$ & $0.4 \pm 0.0$ & $0.08 \pm 0.03$ & $2.50 \pm 0.29$ & $0.73 \pm 0.02$ \\
\hline-25 & 5 & -4 & 26 & 10.3 & $76.4 \pm 0.0$ & $5.92 \pm 0.0$ & $7.02 \pm \quad 0.00$ & $1.29 \pm 0.81$ & $0.4 \pm 0.0$ & $0.09 \pm 0.03$ & $2.50 \pm 0.29$ & $0.72 \pm 0.02$ \\
\hline-30 & 5 & \multicolumn{11}{|c|}{ No data (instrument fiducial) } \\
\hline-40 & 5 & -15 & 42 & 9.3 & $75.4 \pm 1.1$ & $6.91 \pm 0.99$ & $8.65 \pm 1.62$ & $1.12 \pm 1.0$ & $0.6 \pm 0.2$ & $0.07 \pm 0.06$ & $2.20 \pm 0.59$ & $0.69 \pm 0.04$ \\
\hline-45 & 5 & -19 & 47 & 9.0 & $75.1 \pm 0.0$ & $7.90 \pm 0.0$ & $10.3 \pm 0.00$ & $1.12 \pm 0.61$ & $0.8 \pm 0.0$ & $0.09 \pm 0.05$ & $1.75 \pm 0.14$ & $0.87 \pm 0.01$ \\
\hline-55 & 5 & -31 & 60 & 8.3 & $75.5 \pm 0.9$ & $6.91 \pm 0.99$ & $14.7 \pm 0.67$ & $2.18 \pm 1.75$ & $1.0 \pm 0.2$ & $0.12 \pm 0.1$ & $2.20 \pm 0.59$ & $0.95 \pm 0.04$ \\
\hline-60 & 5 & -36 & 66 & 8.0 & $75.3 \pm 0.5$ & $7.30 \pm 0.59$ & $19.4 \pm 1.10$ & $3.55 \pm 2.12$ & $1.4 \pm 0.2$ & $0.24 \pm 0.13$ & $2.20 \pm 0.59$ & $1.2 \pm 0.04$ \\
\hline-70 & 5 & \multicolumn{11}{|c|}{ no data (forshortened southern pole, terminator) --no data (forshortene southern pole, terminator) } \\
\hline \multirow{2}{*}{\multicolumn{4}{|c|}{$\begin{array}{l}\text { 01/27/2011 } \\
\text { ERVATION }\end{array}$}} & \multicolumn{2}{|c|}{$\mathbf{N S O}_{2}(\mu \mathrm{m}-\mathrm{atm})$} & \multicolumn{2}{|c|}{ NSO ( $\mu$ m-atm) } & \multirow{2}{*}{\multicolumn{2}{|c|}{$\mathrm{SO}_{2}$ VMR (ppb) }} & \multicolumn{3}{|c|}{ SO VMR (ppb) } \\
\hline & & & & range & average & range & Average & & & \multicolumn{3}{|c|}{ average } \\
\hline \multicolumn{4}{|c|}{ dayside density } & $0.6 \pm 0.2$ & $0.5 \pm 0.04$ & $0.08 \pm 0.01$ & $0.08 \pm 0.04$ & \multicolumn{2}{|c|}{$7.9 \pm 0.4$} & & $1.2 \pm 0.8$ & \\
\hline \multicolumn{4}{|c|}{ terminator density } & $0.9 \pm 0.5$ & $0.9 \pm 0.15$ & $0.15 \pm 0.09$ & $0.14 \pm 0.09$ & \multicolumn{2}{|c|}{$13.0 \pm 0.8$} & \multicolumn{3}{|c|}{$2.2 \pm 1.4$} \\
\hline
\end{tabular}

(Table III is embedded in the manuscript) 
TABLE V

\begin{tabular}{|c|c|c|c|}
\hline \multicolumn{4}{|c|}{ SOIR OBSERVATIONS (averaged 2006-2013) } \\
\hline \multirow[t]{2}{*}{ ALTITUDE } & \multicolumn{3}{|c|}{ 0-40 ${ }^{\circ}$ LATITUDE } \\
\hline & \multicolumn{2}{|c|}{$\mathrm{SO}_{2} \mathrm{VMR}$ range } & $\mathrm{SO}_{2}$ VMR mid-point ${ }^{\S}$ \\
\hline $81.8 \mathrm{~km}$ & \multicolumn{2}{|c|}{ 40-121 ppb } & $70 \mathrm{ppb}$ \\
\hline $79.7 \mathrm{~km}$ & \multicolumn{2}{|c|}{ 24-133 ppb } & $56 \mathrm{ppb}$ \\
\hline $77.7 \mathrm{~km}$ & \multicolumn{2}{|c|}{ 9-249 ppb } & $47 \mathrm{ppb}$ \\
\hline $75.8 \mathrm{~km}$ & \multicolumn{2}{|c|}{ 11-194 ppb } & $46 \mathrm{ppb}$ \\
\hline $74.9 \mathrm{~km}$ & \multicolumn{2}{|c|}{$13-163$ ppb } & $48 \mathrm{ppb}$ \\
\hline \multicolumn{4}{|c|}{ DECEMBER 28, 2010 COINCIDENT HST \& SOIR OBSERVATIONS } \\
\hline & \multirow{3}{*}{$\begin{array}{c}\text { HST 10-20S } \dagger \\
280(414) \\
\text { ppb }\end{array}$} & \multirow{3}{*}{$\begin{array}{c}\text { HST 20S†† } \\
197(290) \\
\text { ppb }\end{array}$} & SOIR 31S \\
\hline $78 \pm 3.5 \mathrm{~km}$ & & & $290 \pm 12 \mathrm{ppb}$ \\
\hline $77 \pm 3.5 \mathrm{~km}$ & & & $240 \pm 10$ ppb \\
\hline
\end{tabular}

$\S$ The logarithmic midpoint of all values observed at the listed altitude

† The average $\mathrm{SO}_{2}$ VMR expected in the 74-81 km range, assuming 0.965 $\mathrm{CO}_{2}$ abundance, where the average $\mathrm{SO}_{2}$ number density is derived from the number density profile needed to replicate the $\mathrm{SO}_{2}$ column densities fit to the HST observations obtained at $\mathrm{SZA}=60-70^{\circ}$ between latitudes $10-20 \mathrm{~S}$, and the average $\mathrm{CO}_{2}$ number density is derived from the vertical $\mathrm{CO}_{2}$ profile included in the 1985 VIRA catalog. The value in parentheses is the $\mathrm{HST} \mathrm{SO}_{2}$ number density divided by the SOIR retrieved total number density, assuming $0.965 \mathrm{CO}_{2}$ abundance.

$\dagger$ The average $\mathrm{SO}_{2}$ VMR expected between $74-81 \mathrm{~km}$ altitude, assuming $0.965 \mathrm{CO}_{2}$ abundance, where the average $\mathrm{SO}_{2}$ number density is derived from the number density profile needed to replicate the $\mathrm{SO}_{2}$ column densities fit to the HST observations obtained at $\mathrm{SZA}=70^{\circ}$ at $20 \mathrm{~S}$, and the average $\mathrm{CO}_{2}$ number density is derived from the vertical $\mathrm{CO}_{2}$ profile included in the 1985 VIRA catalog. The value in parentheses is the $\mathrm{HST} \mathrm{SO}_{2}$ number density divided by the SOIR retrieved total number density, assuming $0.965 \mathrm{CO}_{2}$ abundance. 


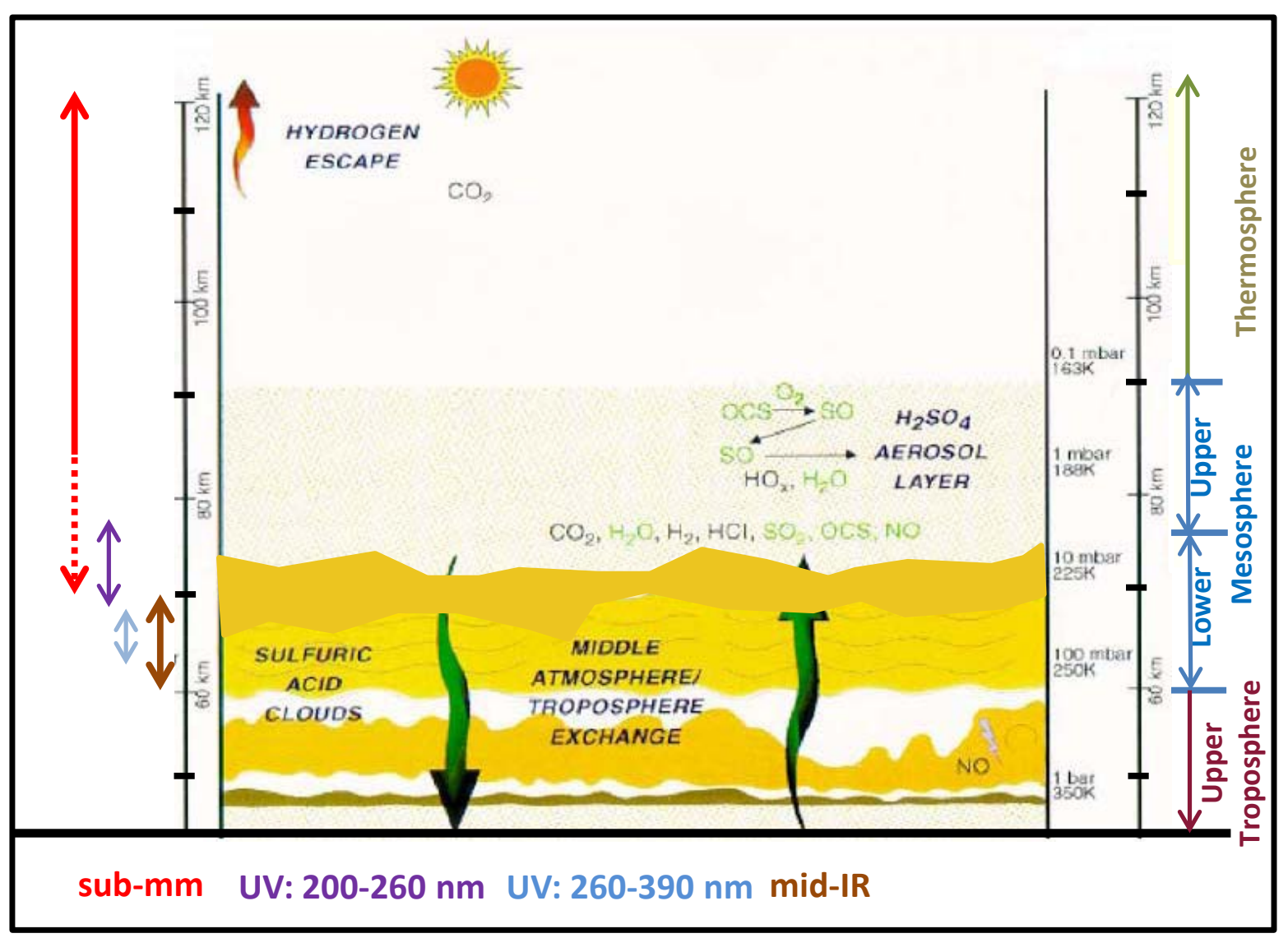

Figure 1.0 


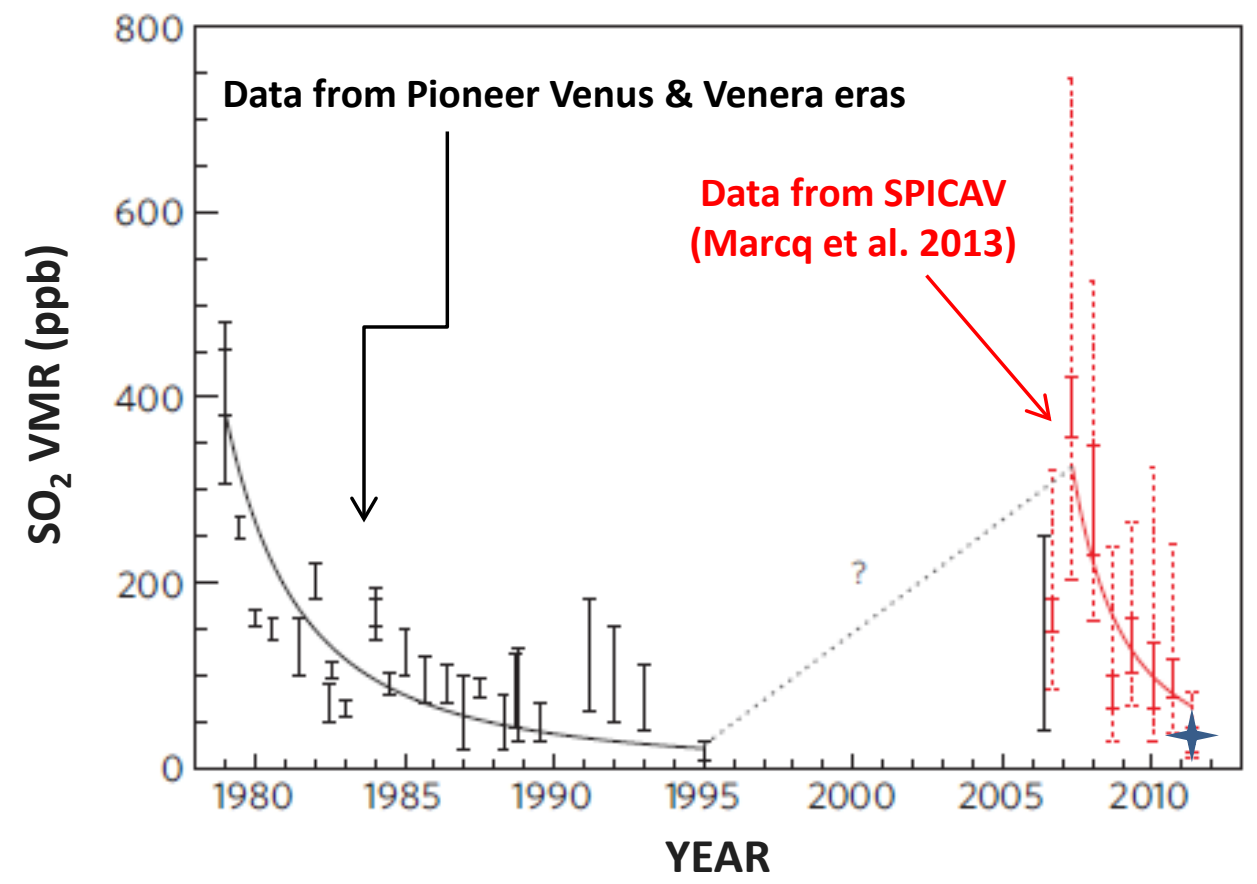

Figure $\mathbf{2 . 0}$ 


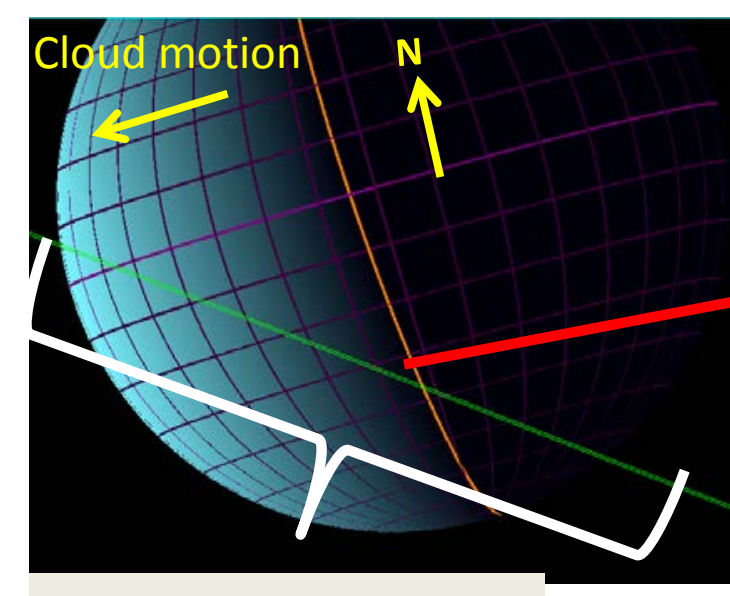

HST/STIS G230LB 0.1" long slit spectrum of Venus

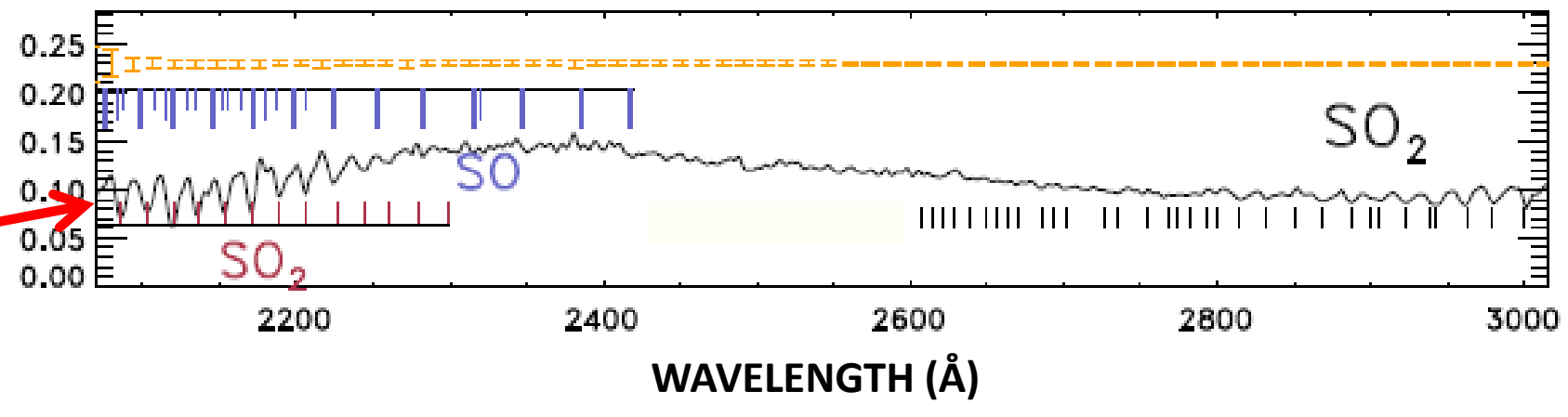

$52 " \times 0.1 "$ HST slit

Figure 3 


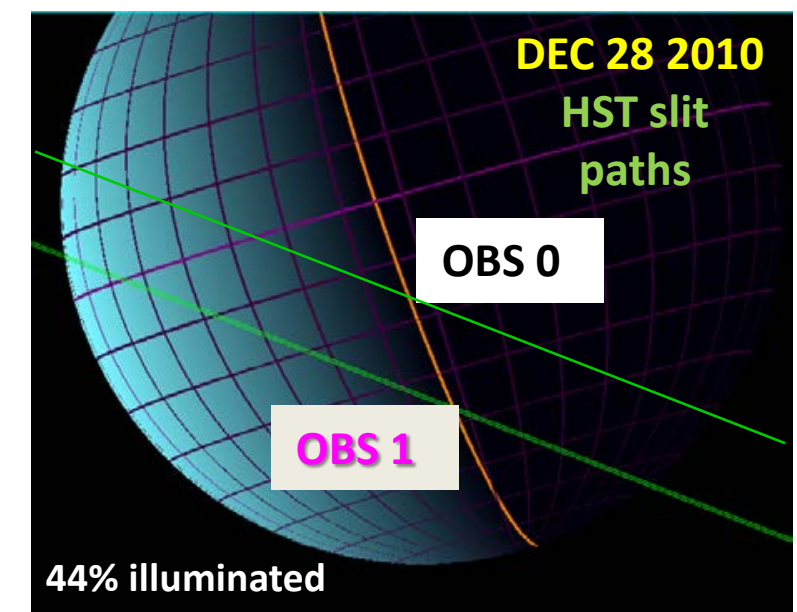

OBS 0: 15S, L65 ${ }^{\circ}$ DEC 28 OBS 1: 32S, L65 ${ }^{\circ}$ DEC 28

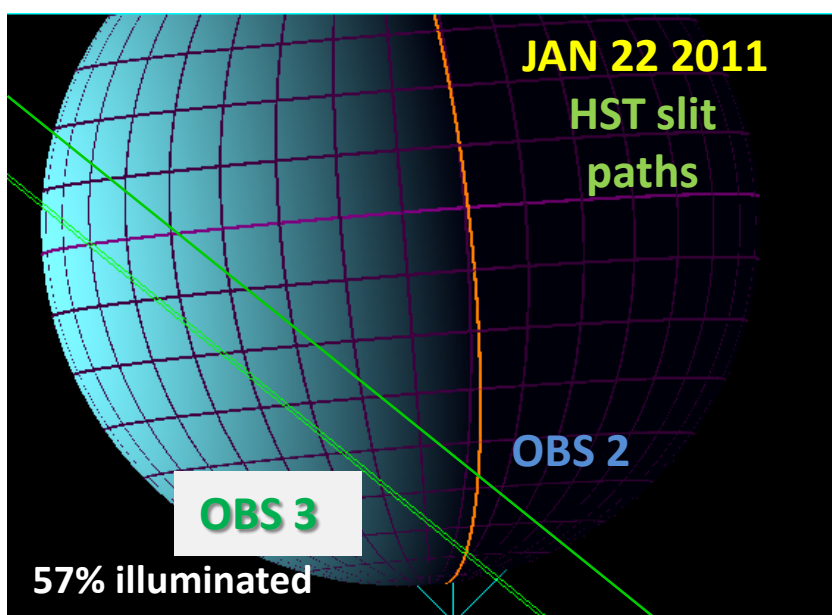

OBS 2: 45S, L145 JAN 22 OBS 3: 65S, L145 JAN 22

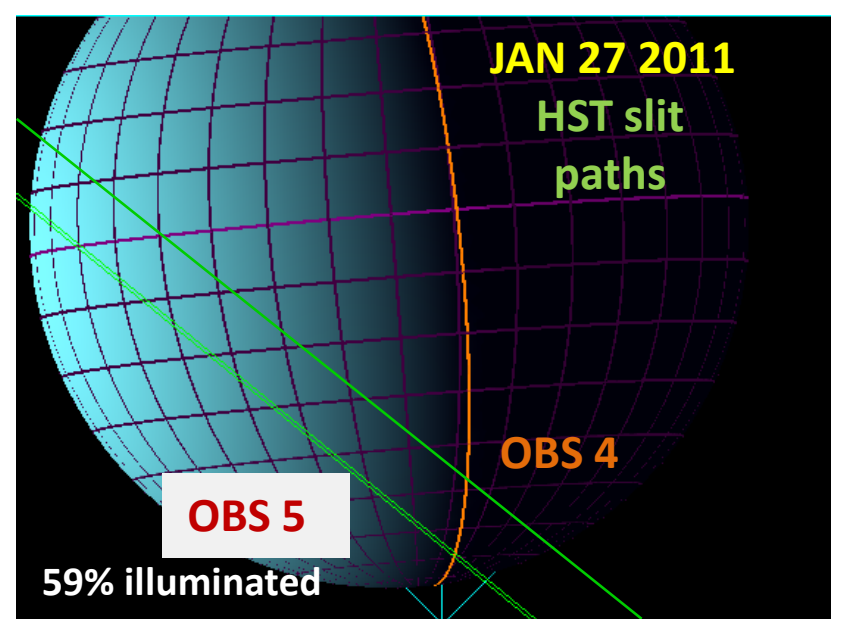

OBS 4: 45S, L160 JAN 27 OBS 5: 65S, L160 JAN 27

Figure 4 

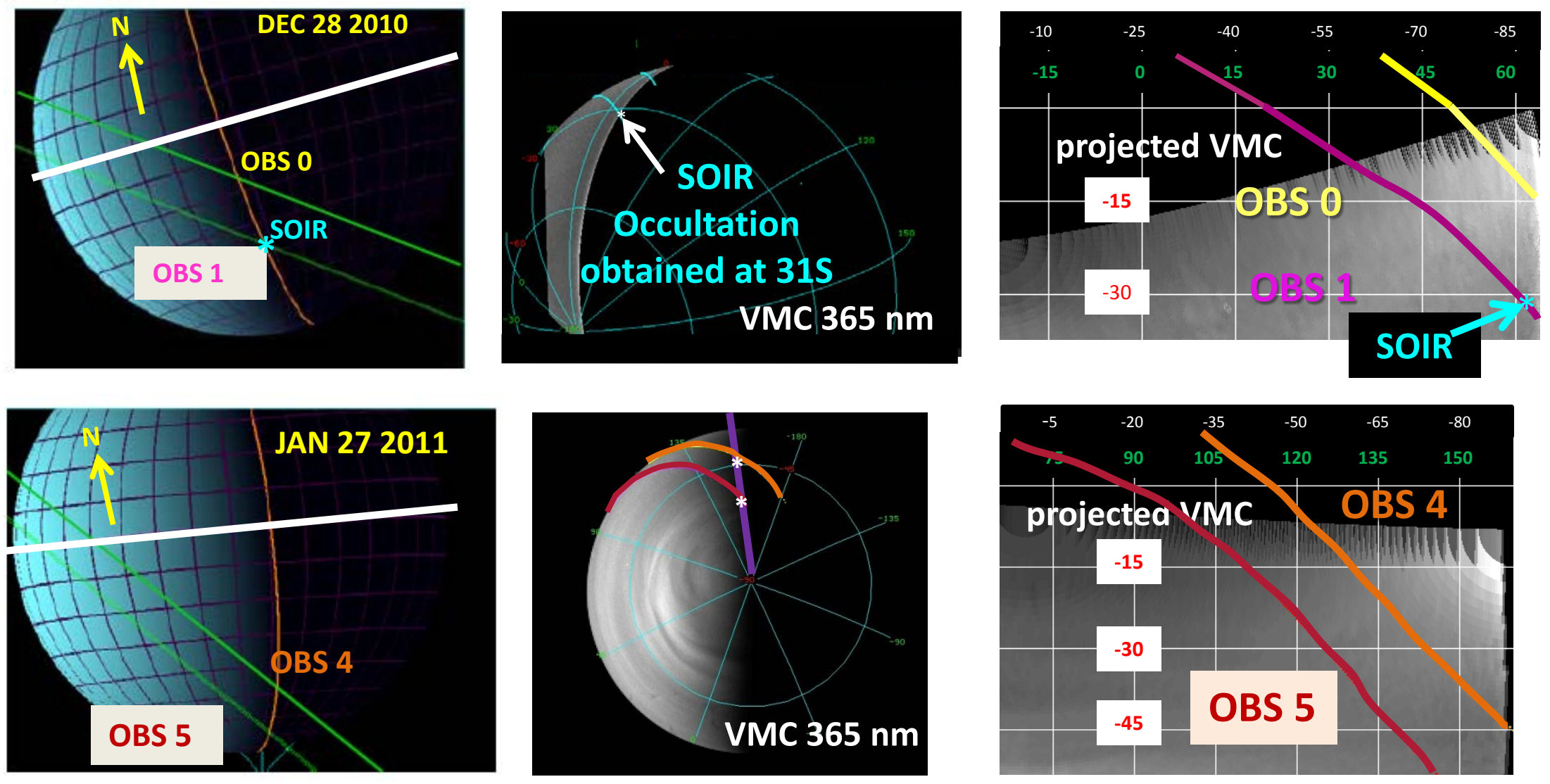

Figure 5 

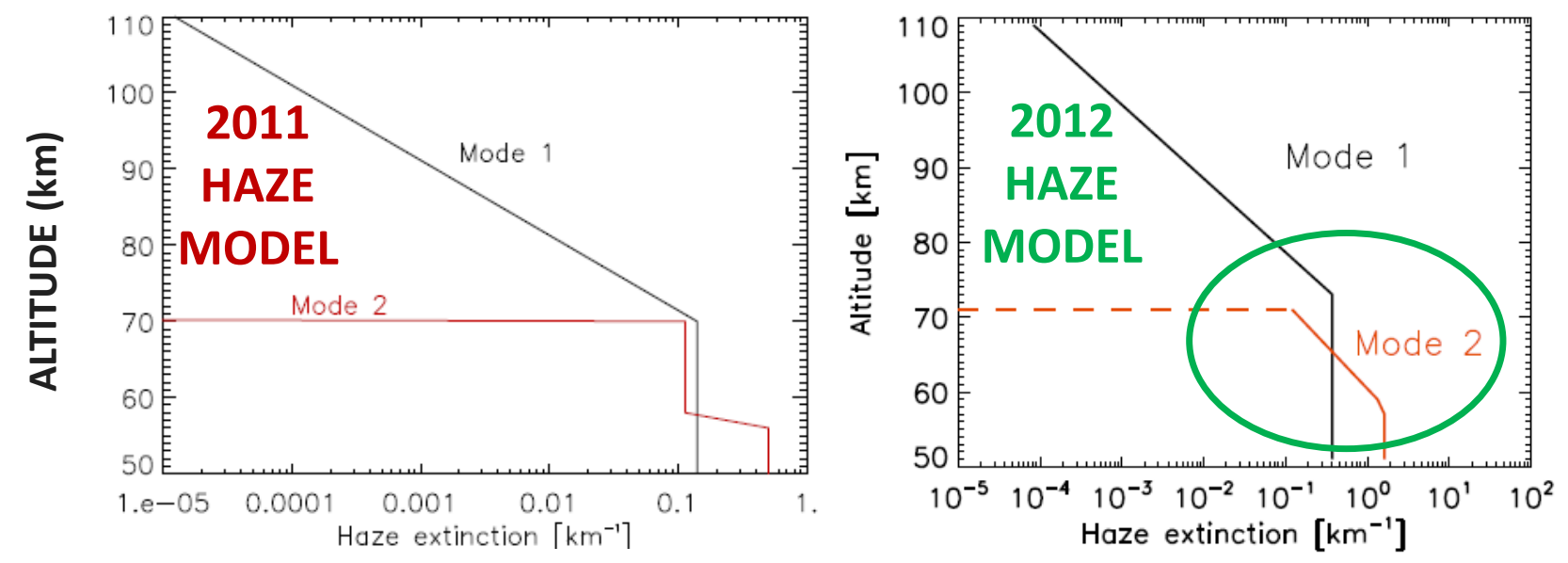

Figure 6 

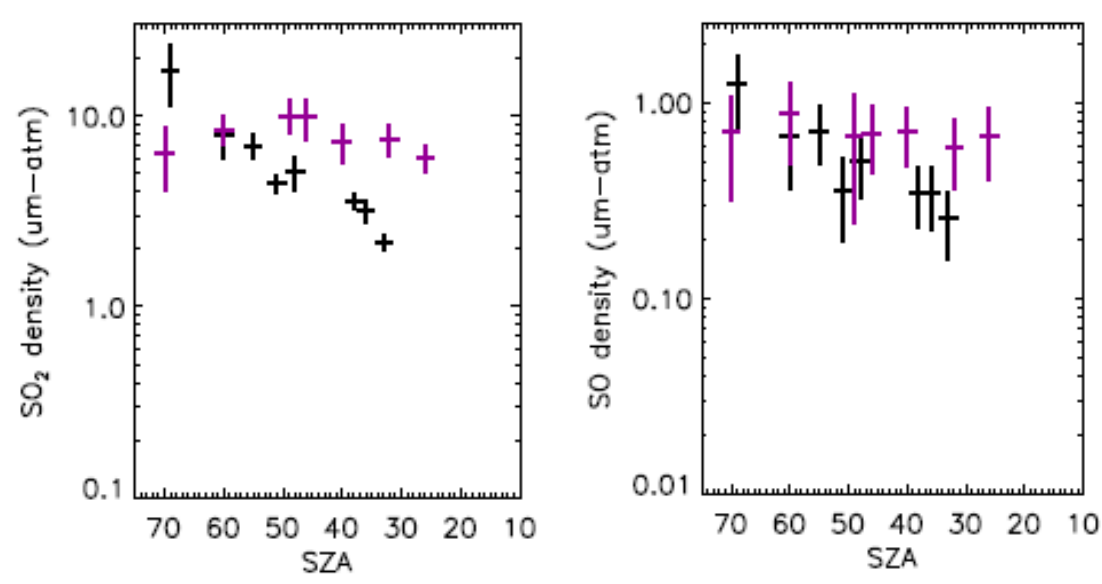

Figure 9
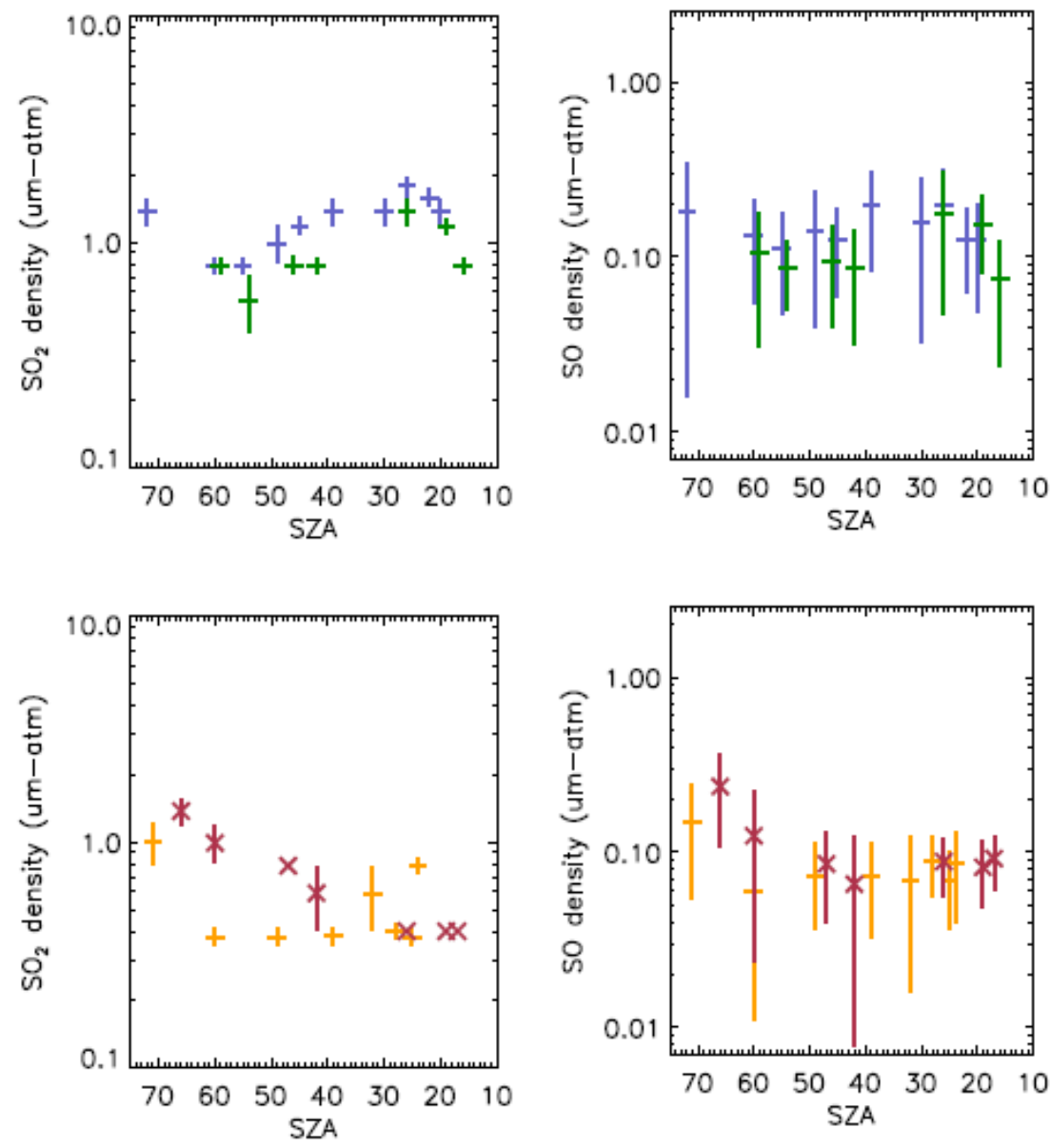


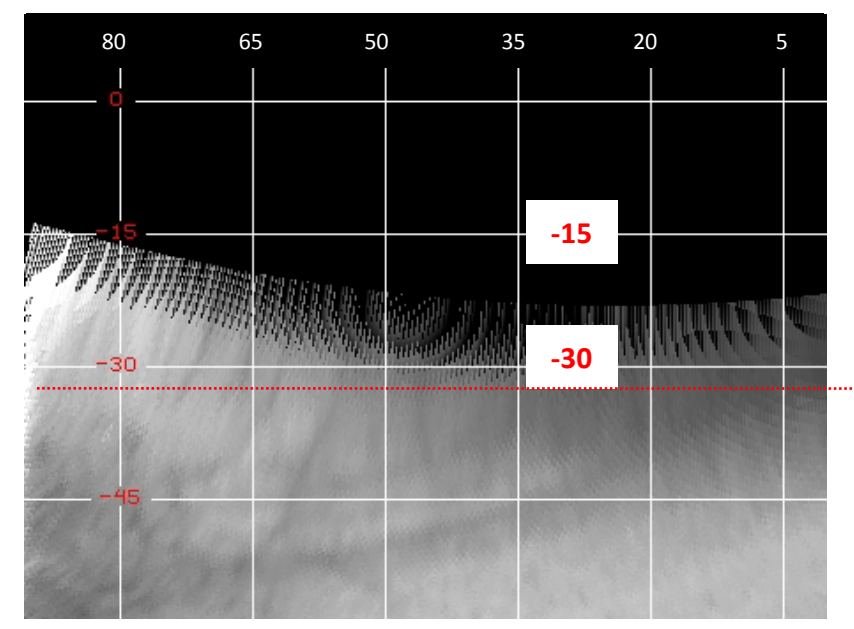

evening quadrant

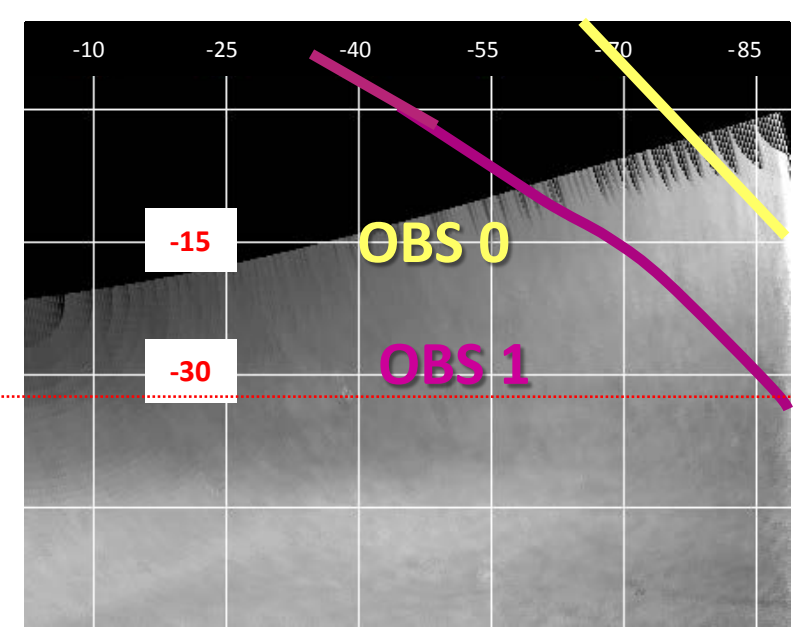

morning quadrant

Figure 10 

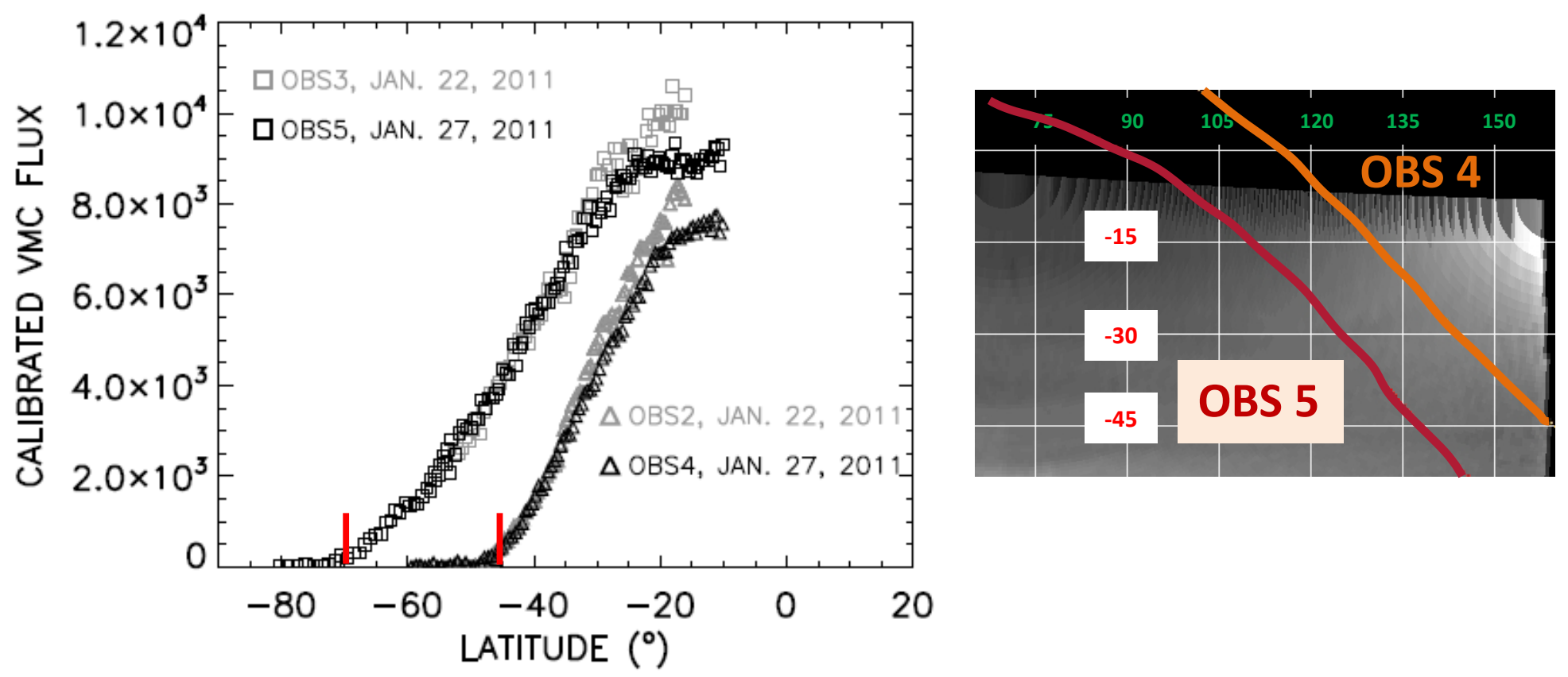

Figure 11 


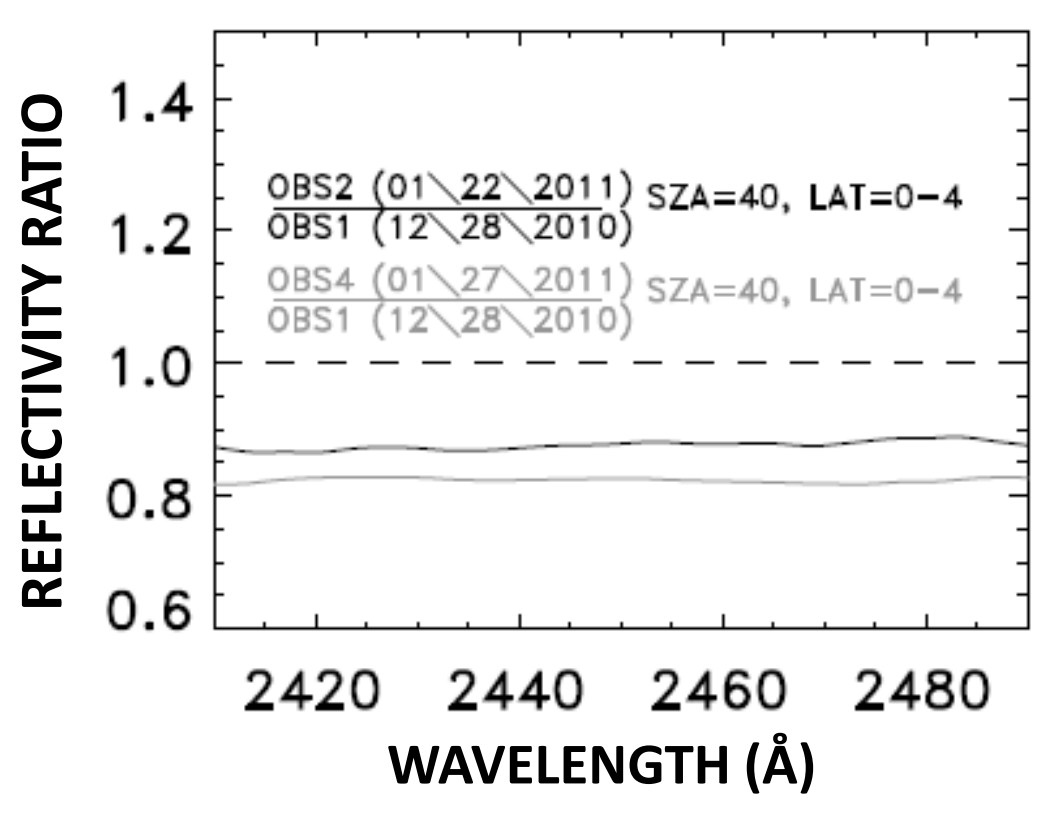

Figure 12

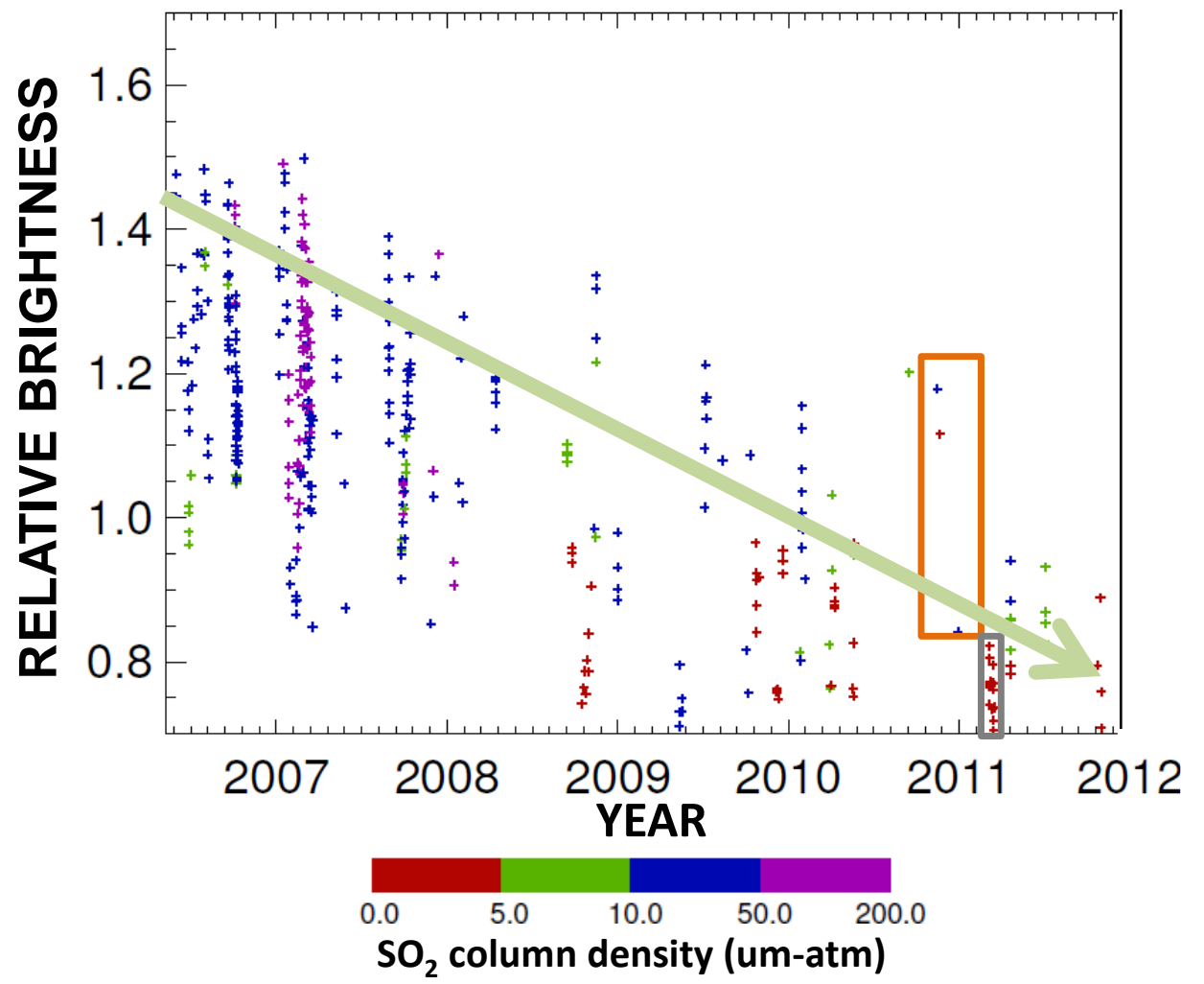



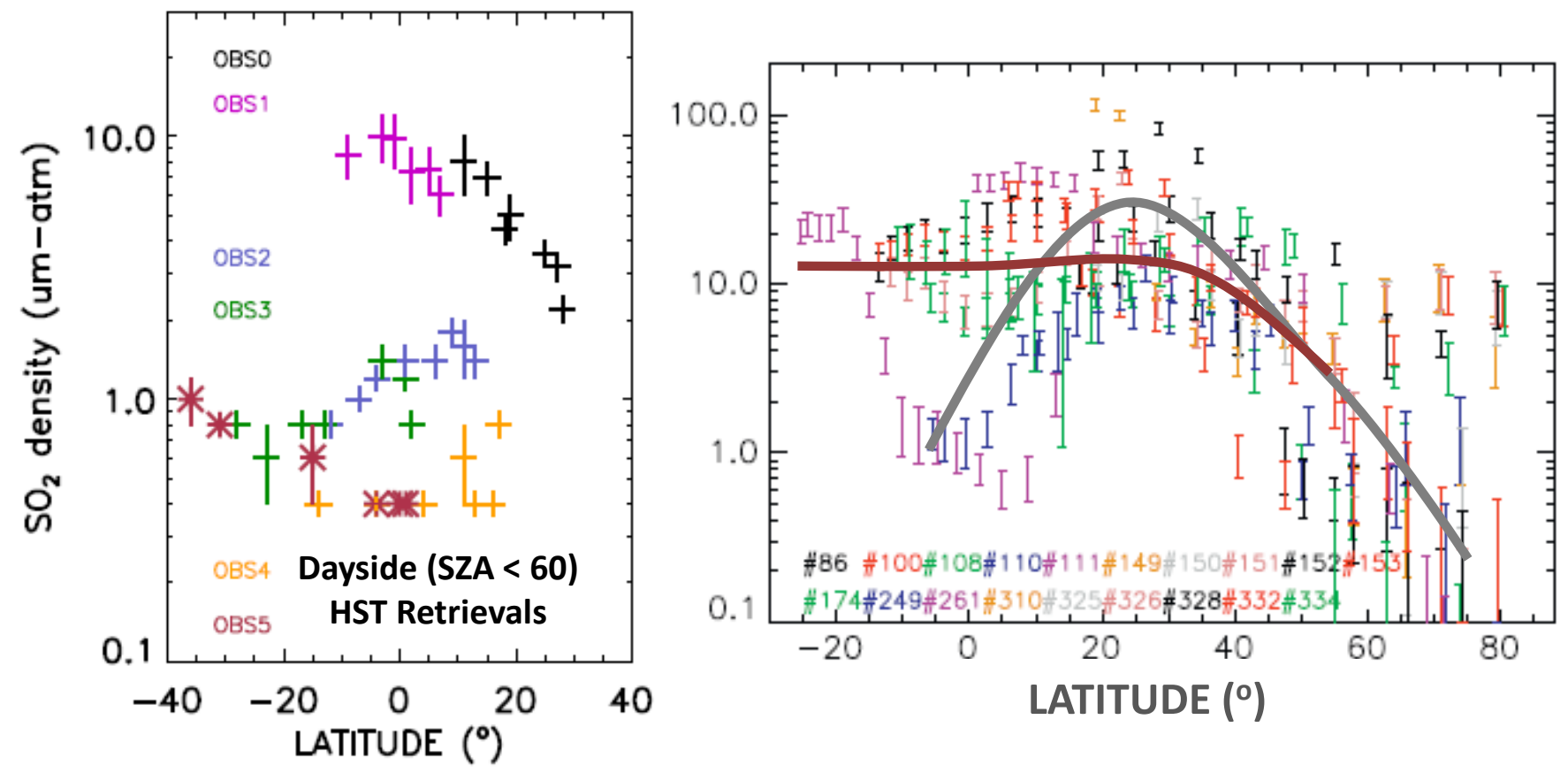

Figure 13 

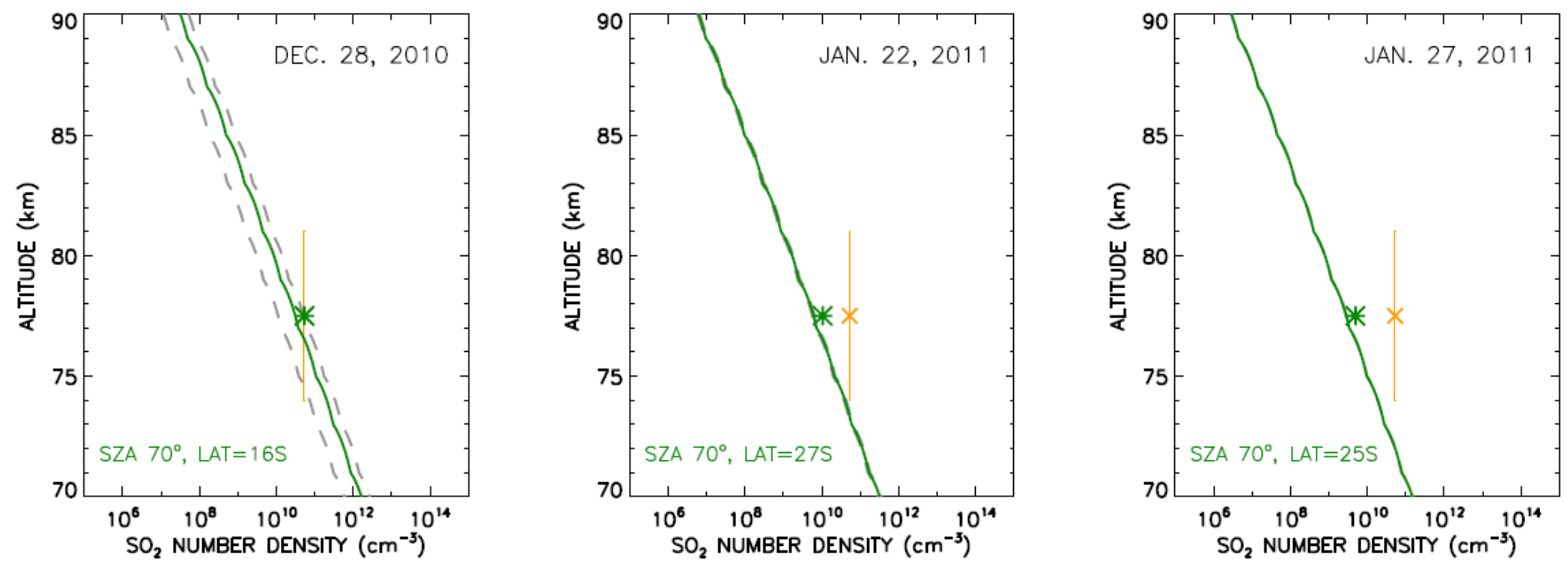

\section{Figure 14}




\section{Figure 15}
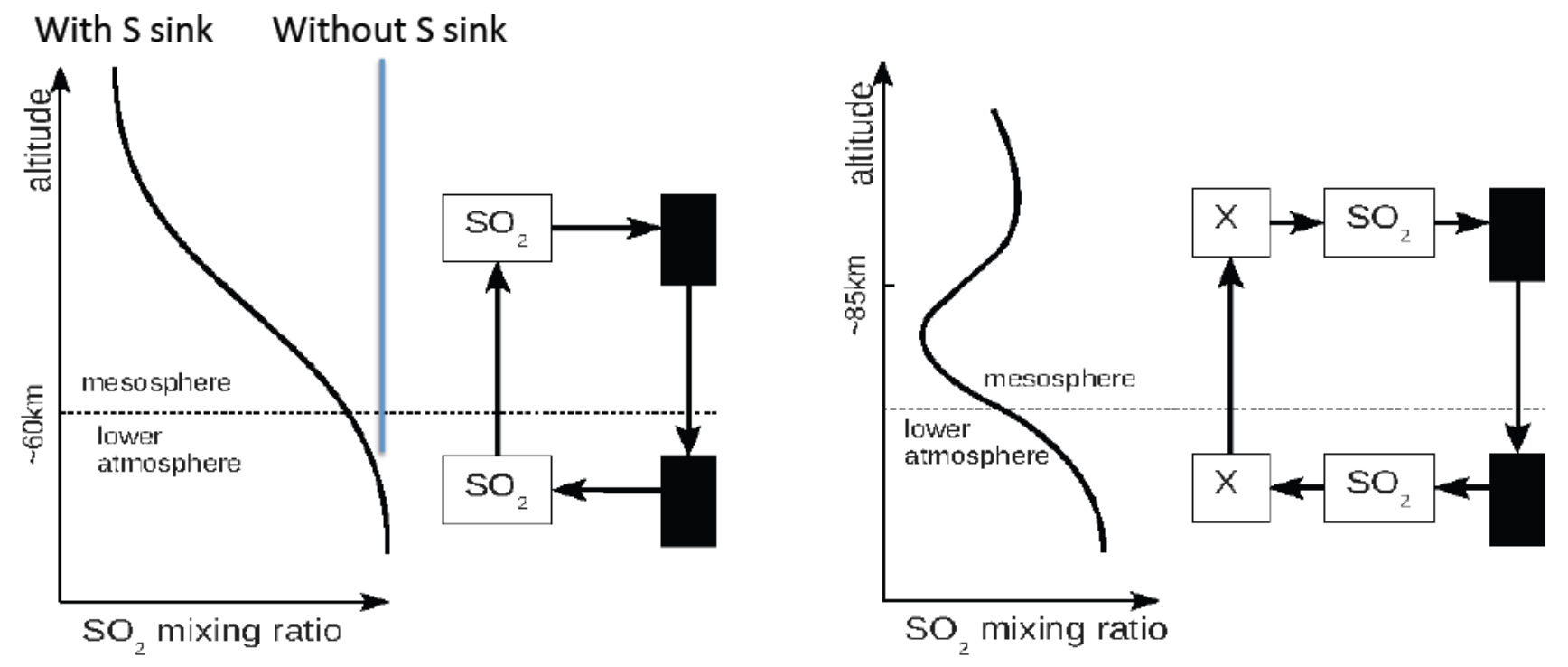

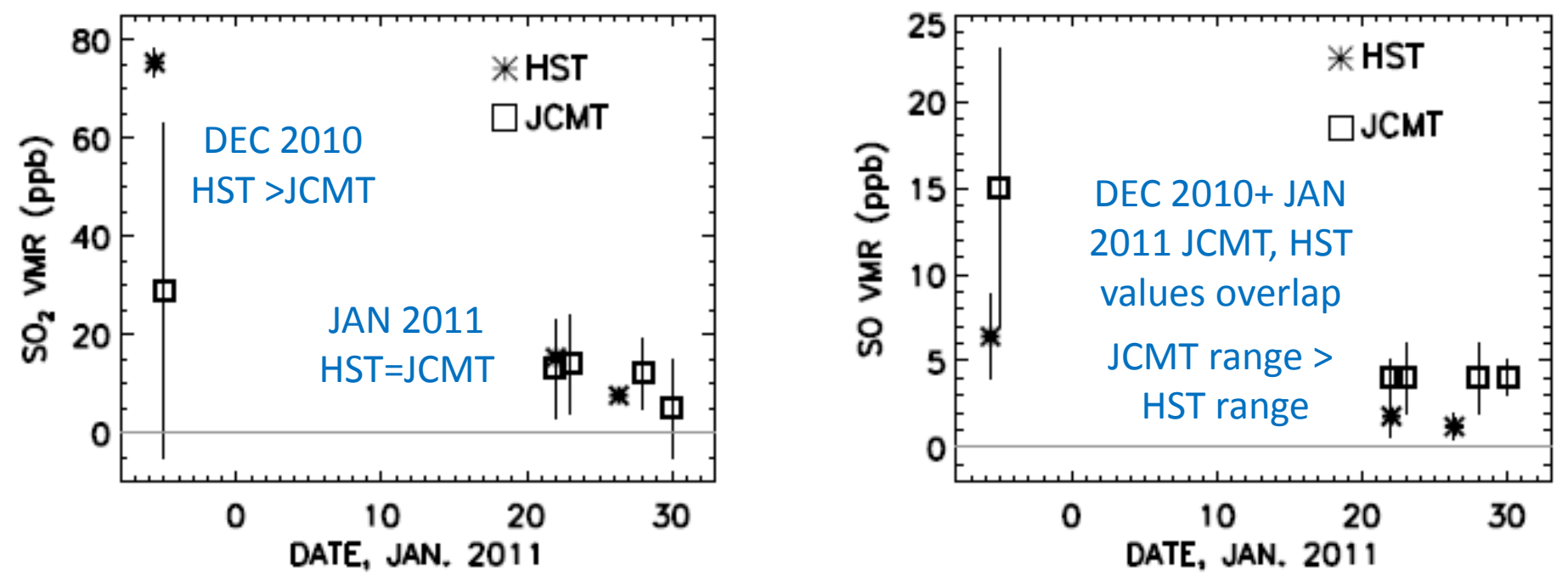

Figure 16 


\section{Figure 17}

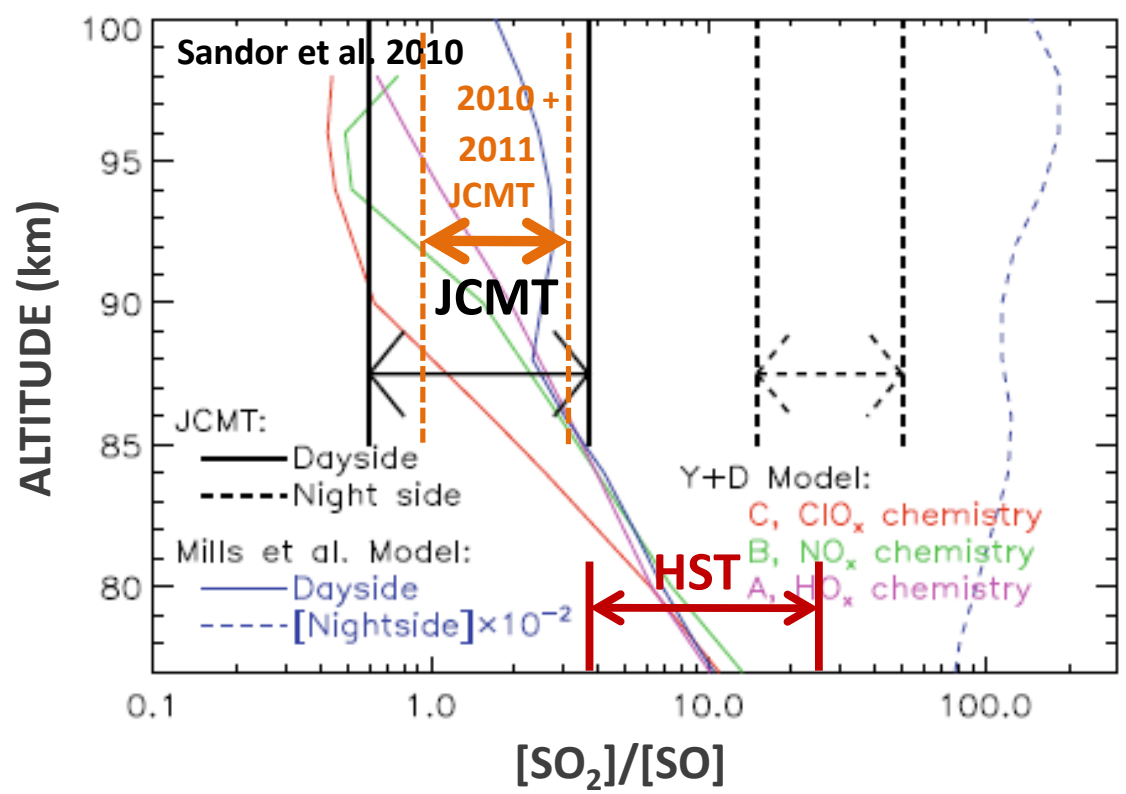



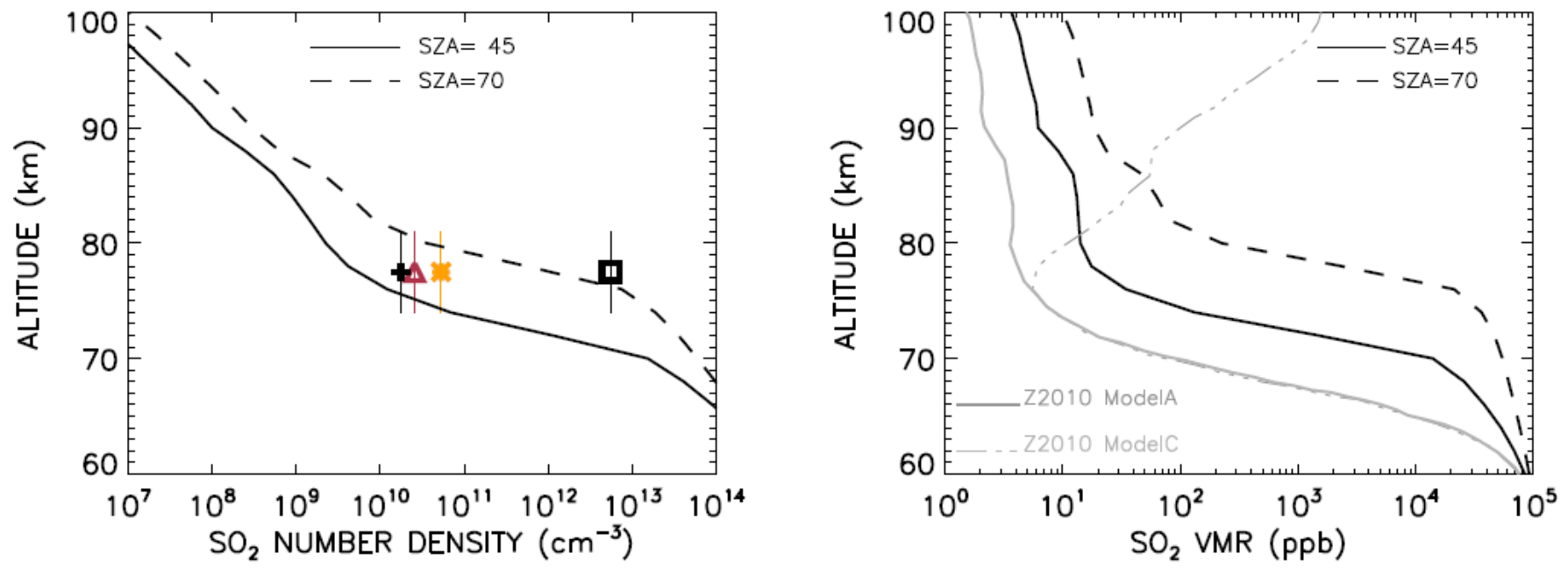

\section{Figure 18}




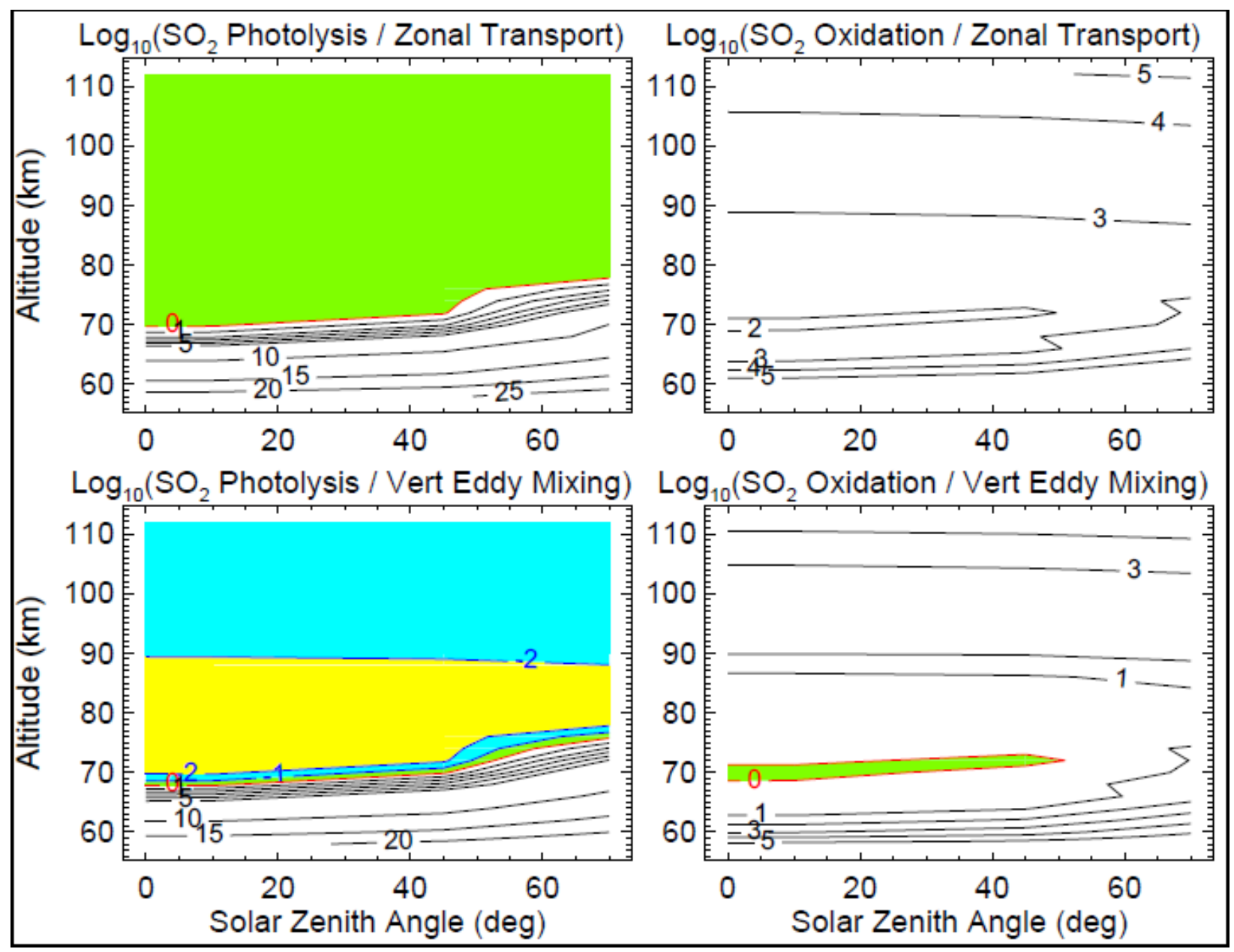

Figure 19 